UCRL-CR-119438

$S / C$ - B235271

\title{
RADIATION EFFECTS IN SPACE: THE CLEMENTINE I MISSION
}

T. Gregory Guzik, Edmund Clayton and John P. Wefel

December 20, 1994

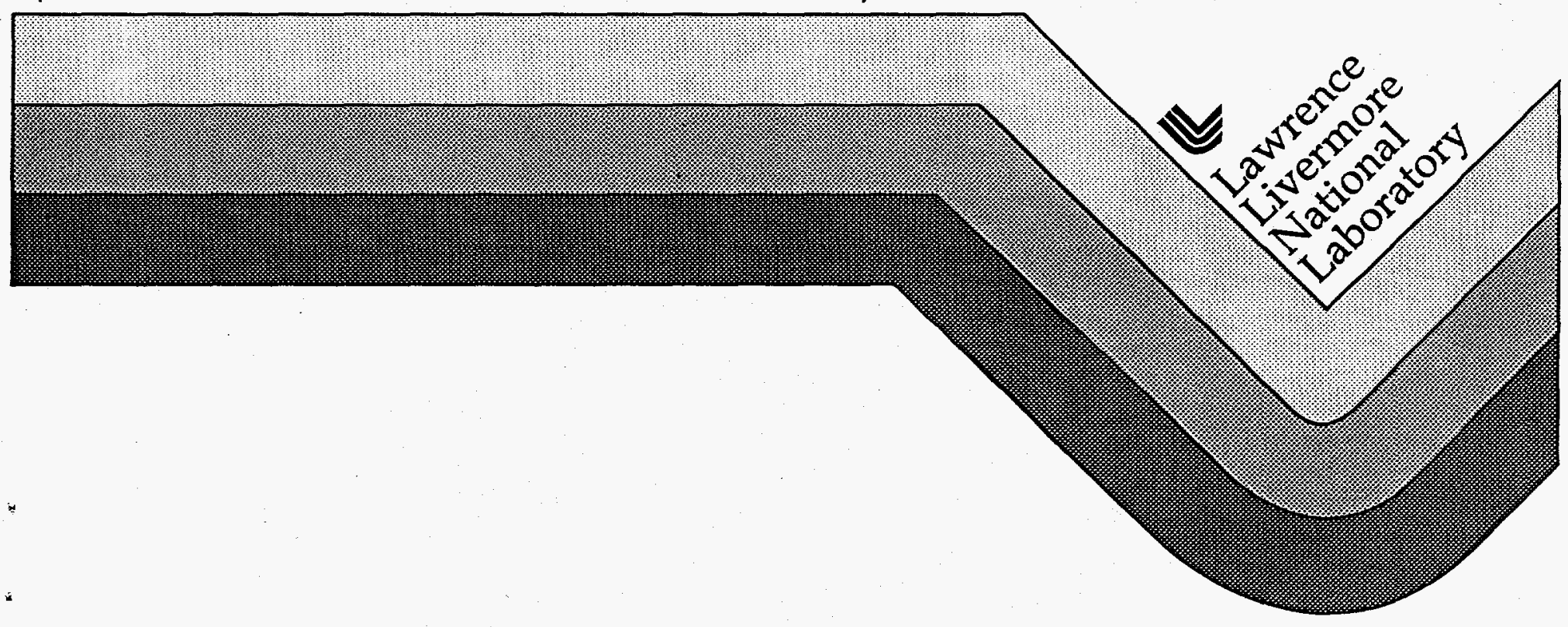

DISTABUTON OF THS DOCUENT IS UNLMITED 


\section{DISCLAIMER}

Work performed under the auspices of the U.S. Department of Energy by Lawrence Livermore National Laboratory under contract number W-7405-ENG-48.

This document was prepared as an account of work sponsored by an agency of the United States Government. Neither the United States Government nor the University of California nor any of their employees, makes any warranty, express or implied, or assumes any legal liability or responsibility for the accuracy, completeness. or usefulness of any information, apparatus, product, or process disclosed, or represents that its use would not infringe privately owned rights. Reference herein to any specific commercial products, process, or service by trade name. trademark, manufacturer. or otherwise, does not necessarily constitute or imply its endorsement, recommendation, or favoring by the United States Government or the University of California. The views and opinions of authors expressed herein do not necessarily state or reflect those of the United States Government or the University of California, and shall not be used for advertising or product endorsement purposes. 


\section{DISCLAIMER}

Portions of this document may be illegible in electronic image products. Images are produced from the best available original document. 


\section{RADIATION EFFECTS IN SPACE: \\ THE CLEMENTINE-1 MISSION}

by:

T. Gregory Guzik

Edmund Clayton

John P. Wefel

Louisiana State University

Department of Physics and Astronomy

Baton Rouge, LA 70803-4001

September, 1994

Sub-Contract B235271

prepared for

University of California

Lawrence Livermore National Laboratory

"O" Division

Livermore, CA 94551 
The space radiation environment for the CLEMENTINE I mission was investigated using a new calculational model, $\mathrm{CHIME}$, which includes the effects of galactic cosmic rays (GCR), anomalous component (AC) species and solar energetic particle (SEP) events and their variations as a function of time. Unlike most previous radiation environment models, CHIME is based upon physical theory and is "calibrated" with energetic particle measurements made over the last two decades. Thus, CHIME provides an advance in the accuracy of estimating the interplanetary radiation environment. Using this model we have calculated particle energy spectra, fluences and linear energy transfer (LET) spectra for all three major components of the CLEMENTINE I mission during 1994: 1) the spacecraft in lunar orbit, 2) the spacecraft during asteroid flyby, and 3) the interstage adapter (ISA) in Earth orbit. Our investigations indicate that during 1994 the level of solar modulation, which dominates the variation in the GCR and AC flux as a function of time, will be decreasing toward solar minimum levels. Consequentlý, the GCR and AC flux will be increasing during the year and, potentially, will rise to levels seen during previous solar minimums (e.g. 1987, 1973-1978, 1965). The estimated radiation environment also indicates that the $A C$ will dominate the energetic particle spectra for energies below $30-50 \mathrm{MeV} /$ nucleon, while the GCR have a peak flux at -300 $\mathrm{MeV} /$ nucleon and maintain a relatively high flux level up to $>1000$ $\mathrm{MeV} /$ nucleon. The AC significantly enhances the integrated flux for LET in the range 1 to $10 \mathrm{MeV} /\left(\mathrm{mg} / \mathrm{cm}^{2}\right)$, but due to the steep energy spectra of the $\mathrm{AC} \mathrm{a}$ relatively small amount of material ( $\sim 50$ mils of $\mathrm{Al}$ ) can effectively shield against this component. The GCR are seen to be highly penetrating and require massive amounts of shielding before there is any appreciable decrease in the LET flux. For SEP events, we have extrapolated from the historical record to estimate that $10 \pm 5$ events might be observed during 1994 and the total fluence of $>10 \mathrm{MeV}$ protons for these events should be less than $10^{8}$ particles $/\left(\mathrm{cm}^{2} \mathrm{sr}\right)$. Four samples of 10 events, selected from those included in the CHIME model, were used to investigate the SEP effects on the LET spectrum. Each sample had a different composition and were labeled 1) typical, 2) heavy ion rich, 3) heavy ion poor and 4) typical with a single large event. Slight differences were found for samples $1-3$ for LET $>3-4 \mathrm{MeV} /\left(\mathrm{mg} / \mathrm{cm}^{2}\right)$ due to differences in the heavy ion content, while the single large event in sample 4 resulted in significant fluence enhancements for all LET values. LET spectra for all three components of the CLEMENTINE I mission were calculated by combining the GCR, AC and "typical" composition SEP fluxes and, for the ISA, taking into account the effect of geomagnetic transmission. It was found that GCR dominate the LET spectra for LET greater than about $1 \mathrm{MeV} /\left(\mathrm{mg} / \mathrm{cm}^{2}\right)$ and below this level the SEP effects can be seen for Al shield less than 300 mils thick. The effect of geomagnetic transmission of the interplanetary radiation averaged over the ISA orbit appears to be negligible. Plots and tables of estimated LET spectra are provided in this report. 
ABSTRACT ii

LIST OF FIGURES iv

LIST OF TABLES. $\mathbf{x i}$

I. INTRODUCTION 1

II. THE ENERGETIC PARTICLES. .2

A. Galactic Cosmic Rays. 3

B. Anomalous Component.

C. Solar Energetic Particles . .10

III. THE CHIME ENERGETIC PARTICLE MODEL .15

A. Scope and Functionality. 15

B. The Physical Basis of CHIME

1. The Local Interstellar Particle Spectra ......................................................17

2. Solar Modulation ..............................................................................34

3. Solar Energetic Particle Events .............................................................39

4. Geomagnetic Transmission ....................................................................44

5. The Linear Energy Transfer Spectrum Calculator..................................58

IV. THE CLEMENTINE RADIATION ENVIRONMENT …................................59

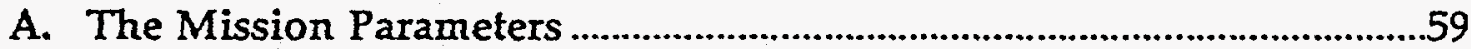

B. Expected Solar Modulation Levels during Mission...................................61

1. GCR Spectrum Variation.........................................................................61

2. Anomalous Component Variation...........................................................63

C. Expected SEP Events during Mission ..........................................................63

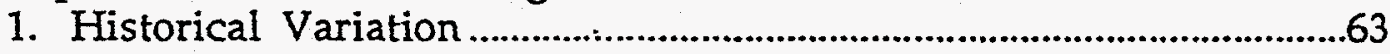

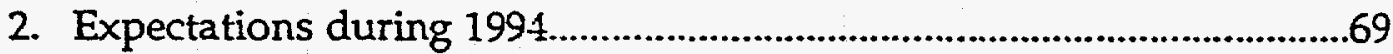

D. The Geomagnetic Transmission of Interplanetary Particles....................70

E. The Linear Energy Transfer (LET) Functions...............................................75

1. Effects of Detector and Shield Thickness .................................................75

2. Interplanetary phases (Lunar and Geographos).................................78

3. Geomagnetic Effects and the Interstage Adapter ................................85

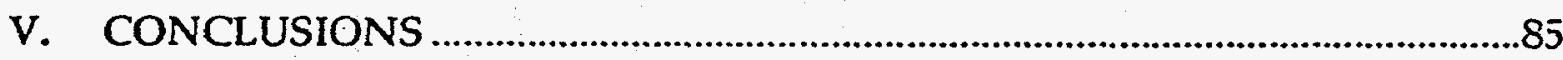

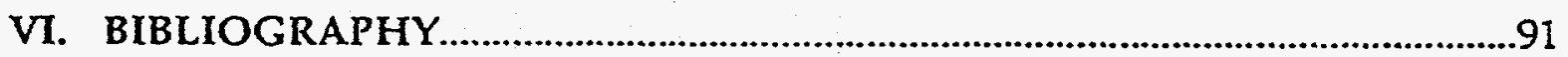




\section{LIST OF FIGURES}

Figure 1: The relative elemental abundances for Solar System material (open circles and dashed line), Local Galactic matter (open bars), and the Cosmic Rays measured near Earth (filled circles and solid lines).

Figure 2: Compiled measurements of the differential energy spectrum for Cosmic Ray H, He, C and Fe made near Earth, mostly during solar minimum conditions. At high energies, the spectral shape is a power law with an index of about -2.7. For energies below $\sim 2000 \mathrm{MeV} /$ nucleon solar modulation causes the spectra to turn over giving the peak cosmic ray flux at $400-600 \mathrm{MeV} /$ nucleon. The flattening in the He spectrum below $100 \mathrm{MeV} /$ nucleon is due to the addition of the Anomalous Component.

Figure 3: Monthly averaged Climax neutron monitor measurements from 1953 to 1993 . During solar minimum conditions the cosmic ray flux is enhanced while during solar maximum conditions the cosmic ray flux is repressed. Note that the last solar maximum (1989-1992) was the most intense maximum observed since 1953.

Figure 4a,b: Low energy cosmic ray measurements of (a) Hydrogen and (b) Helium for solar minimum conditions. .7

Figure 4c-f: Low energy cosmic ray measurements at solar minimum, and at various locations in the heliosphere, for: (c) $C, N$ and $\mathrm{O}$ at 1 A.U., (d) $\mathrm{O}$ at 1 and 15 A.U. (e) $\mathrm{Ne}$ and $\mathrm{Ar}$ at 1 A.U., and $(f) \mathrm{Mg}, \mathrm{Si}, \mathrm{S}$ and $\mathrm{Fe}$ 8

Figure 5: Low energy (8-27 MeV/nudeon) Anomalous Oxygen measured near Earth (data points) from 1972 to 1988 compared with the scaled value of the Mt. Washington neutron monitor rate (solid line)

Figure 6: Proton and Helium flux measurements for an "average" flare (Event 26, panel $A$ ) and the largest flare (Event 10, panel B) seen during 1991. The proton measurements (filled circles) were obtained from GOES and the Helium flux points (open squares) are from the ONR-604 instrument on-board the CRRES spacecraft. The curves are power law fits to the data, and the parameters for these spectra are given in Tables $2-4$. 
Figure 7: The relative enhancement of SEP even- $Z$ elemental abundances over "solar system" composition versus the charge of the element. The enhancements are normalized to Oxygen $=1$.

Figure 8: The mean monthly sunspot number (solid line) from 1950 to 1993 and the yearly number of SEP events observed by instruments on the GOES and IMP-8 spacecraft from 1976 to 1993 (dashed line).

Figure 9: The total fluence (particles/ $\mathrm{cm}^{2}$-sr) of Solar Energetic

Protons with energy $>10 \mathrm{MeV}$ for each year from 1956 to 1993. Prior to 1976 the proton fluence is determined from the Feynman et al. (1993) model and after 1976 from GOES/IMP-8 measurements.

Figure 10: Flow chart of the Cosmic Ray Model developed for the CRRES/

SPACERAD mission and used in evaluating the

CLEMENTINE radiation environment.

Figure 11: Schematic drawing of the transport of cosmic ray particles from the "source" to the orbit of Earth where they are observed.

Figure 12: Measurements of the secondary to primary ratios $B / C$ (top) and ( $\mathrm{Sc}+\mathrm{Ti}+\mathrm{V}+\mathrm{Cr}+\mathrm{Mn}$ )/ $\mathrm{Fe}$ (bottom) compared with the results of interstellar transport and solar modulation calculations.

Figure 13: The pathlength distribution (PLD) parameters used in fitting the $\mathrm{B} / \mathrm{C}$ and Sub-Fe/ $\mathrm{Fe}$ measurements. An exponential distribution of pathlengths with characteristic $X_{0}$ (solid curve) was used along with requiring the cosmic rays to pass through at least $X_{c}$ (dashed curve) amount of material.

Figure 14: Differential energy spectra for the elements $\mathrm{H}, \mathrm{He}, \mathrm{Be}$ and $B$ (see text for details).

Figure 14 (cont): Differential energy spectra for the elements $C, N, O$ and $F$ (see text for details) .24

Figure 14 (cont): Differential energy spectra for the elements $\mathrm{Ne}, \mathrm{Na}$, $\mathrm{Mg}$ and $\mathrm{Al}$ (see text for details). 
Figure 14 (cont): Differential energy spectra for the elements $\mathrm{Si}, \mathrm{P}, \mathrm{S}$ and $\mathrm{Cl}$ (see text for details).

Figure 14 (cont): Differential energy spectra for the elements Ar, P, Ca and $\mathrm{Sc}$ (see text for details).

Figure 14 (cont): Differential energy spectra for the elements $\mathrm{Ti}, \mathrm{V}, \mathrm{Cr}$ and $\mathrm{Mn}$ (see text for details). 28

Figure 14 (cont): Differential energy spectra for the elements $\mathrm{Fe}, \mathrm{Co}$ and $\mathrm{Ni}$ (see text for details).

Figure 15: The Anomalous Component local interstellar spectra for $\mathrm{He}, \mathrm{N}, \mathrm{O}$ and $\mathrm{Ne}$ used in the Model. The Helium and Oxygen spectra were both derived by demodulating anomalous component measurements, while the LIS for $\mathrm{N}$ and Ne were obtained by scaling the Oxygen spectra.

Figure 16: Anomalous Oxygen measurements (filled circles) made at different times and at different locations in the heliosphere compared to the GCR Model calculations (solid lines). From left to right the measurements were made by IMP-8 at I A.U. during 1977, by Voyager I \& II at 2 A.U. during $9 / 1977-2 / 1978$, by Pioneer 10 at 15 A.U. during $1 / 1977-3 / 1978$, and by Voyager II at 21 A.U. during 10/1985 - 8/1986. Each calculated spectrum used the same LIS, but with heliospheric radii and solar modulation level $(\phi)$ appropriate to the given data. $32, \cdots$

Figure 17: Similar to Figure 16, but for anomalous Nitrogen and Neon. Measurements were made by Voyager I \& II at 2 A.U. during $9 / 1977-2 / 1978$ and by Voyager II at 21 A.U. during 10/1985-8/1986.

Figure 18: Daily average flux of $70-95 \mathrm{MeV} /$ nucleon Helium as measured by the IMP-8 satellite from 1973 to 1993 . The solar cycle variation is clearly seen as the broad, dark band with solar energetic particle (SEP) active times (sharp spikes) superimposed. Very few SEP events occur during solar minimum periods, but can occur for several years after solar maximum. Note that during 1992 and 1993 the quiet time Helium flux is returning to a solar minimum level. This trend is expected to continue during. 1994. .36

Figure 19: The time variation of solar modulation from 1973 to 1993. The top panel shows the Climax neutron monitor rate 
which tracks with the monthly averaged 70-95

$\mathrm{MeV} /$ nucleon IMP-8 Helium, cleaned of SEP active times, (middle panel). The solar modulation parameter $(\phi)$ derived from the Helium flux is shown in the bottom panel. For solar minimum $\phi$ is about $450 \mathrm{MV}$, while for solar maximum $\phi$ can be as large as 1500 to $1600 \mathrm{MV}$.

Figure 20: The solar modulation parameter, $\phi$, used in the GCR Model covering the period 1970 to 2010 . The $\phi$ derived from the IMP-8 Helium flux is used for the period 1973 to 1993. Smoothing this data and translating it 22 years (one complete solar cycle) forward and backward in time provides a prediction for the extended period. Note that this method predicts a solar minimum period lasting from -1995 to 2000 .

Figure 21: Spectra and elemental abundance characteristics for SEP events \#1 and \#2 (see text for details).

Figure 21 (cont.): Spectra and elemental abundance characteristics for SEP events \#3 and \#4 (see text for details). 46

Figure 21 (cont.): Spectra and elemental abundance characteristics for SEP events \#5 and \#6 (see text for details).

Figure 21 (cont.): Spectra and elemental abundance characteristics for SEP events \#7 and \#8 (see text for details).

Figure 21 (cont.): Spectra and elemental abundance characteristics for SEP events \#9 and \#10 (see text for details).

Figure 21 (cont.): Spectra and elemental abundance characteristics for SEP events \#11 and \#12 (see text for details). 50

Figure 21 (cont.): Spectra and elemental abundance characteristics for SEP events \#12 and \#14 (see text for details). 51

Figure 21 (cont.): Spectra and elemental abundance characteristics for SEP events $\# 15$ and $\# 16$ (see text for details).

Figure 21 (cont.): Spectra and elementa! abundance characteristics for SEP events \#17 and \#18 (see text for details) .53

Figure 21 (cont.): Spectra and elemental abundance characteristics for SEP events \#19 and \#20 (see text for details) 
Figure 21 (cont.): Spectra and elemental abundance characteristics for SEP events $\# 21$ and $\# 22$ (see text for details).

Figure 21 (cont.): Spectra and elemental abundance characteristics for SEP events \#23 and \#24 (see text for details) 56

Figure 21 (cont.): Spectra and elemental abundance characteristics for SEP events \#25 and \#26 (see text for details)

Figure 22: The Clementine Lunar survey and Geographos encounter periods (left) and Interstage Adapter (ISA) anticipated orbit (right). This information is used to establish the predicted solar modulation level for the spacecraft and to calculate the geomagnetic transmission for the ISA

Figure 23: The effect of solar modulation during 1994 on the Galactic Cosmic Ray Helium spectrum. The Helium differential energy spectrum predicted for January, 1994 and December, 1994 is shown in panel A. The variation in the spectrum for each month during 1994, relative to January, is shown in panel $B$.

Figure 24: Similar to Figure 23 except for Anomalous Component Helium

Figure 25: The time variation in the number of SEP events. Panel A (upper left) shows a correlation plot of the mean sunspot number versus number of SEP events observed during 1976 to 1993. Panel B shows the number of SEP events per year (dashed curve) inferred from the mean sunspot number (solid curve). A comparison of the observed (solid) and inferred (dashed) number of SEP events is shown in panel $C$ (lower left). Finally, the inferred SEP event number is drawn in panel $D$ (lower right) with the peak number for each cycle (filled inverted triangle) and the number 5 years following the peak (filled circles) marked.

Figure 26: The total yearly fluence (particles $/ \mathrm{cm}^{2}$-sr) for $>10 \mathrm{MeV}$ protons in SEP events between 1976 and 1993 based upon GOES and IMP-8 observations. Each curve is for a particular integral fluence range. The fluence ranges are: $200-10^{4}$ (dot-dash), 104 - $10^{5}$ (dot-dot-dash), $10^{5}-10^{6}$ (long dash), $10^{6}-10^{7}$ (medium dash), $10^{7}-10^{8}$ (short dash), and $>10^{8}$ (solid). 
Figure 27: The number of SEP events within a particular fluence range as a function of time from 1976 to 1993 . The fluence ranges are: $200-10^{4}(\mathrm{~A}), 10^{4}-10^{5}(\mathrm{~B}), 10^{5}-10^{6}(\mathrm{C}), 10^{6}-10^{7}$ (D), $10^{7}-10^{8}(\mathrm{E})$, and $>10^{8}(\mathrm{~F})$.

Figure 28: Fluence spectra and elemental abundance characteristics for SEP Sample 1 - Heavy Ion Poor (top) and SEP Sample 2 - Heavy Ion Rich (bottom)

Figure 28 (cont): Fluence spectra and elemental abundance characteristics for SEP Sample 3 - Typical (top) and SEP Sample 4 - Large (bottom).

Figure 29: The elemental abundances for the 1994 SEP Samples relative to the "Typical" Sample 3 - composition. The solid curve is for the Heavy Ion Poor Sample 1, the short dashed curve is for the Heavy Ion Rich Sample2, and the long dashed curve is for the Large Event Sample 4.

Figure 30. The geomagnetic transmission function calculated for the Clementine Interstage Adapter orbit.

Figure 31. Ratio of particle energy spectra seen in interplanetary. space relative to that averaged over the Interstage Adapter orbit. The solid curve is for protons with $A / Z=1$, the short dashed curve is for Helium and other heavy ions with $\mathrm{A} / \mathrm{Z}=2$, and the long dashed curve is for heavy ions with $2.18<\mathrm{A} / Z<2.40$

Figure 32: The integral Linear Energy Transfer (LET) spectrum of a SEP event for a 1 micron thick Silicon sensitive layer as a function of shield thickness. The curves from the top down are for Aluminum shields that are 1, 50, 100, 200, 400,800 and 1600 mils thick. Note that only a modest amount of shielding will significantly reduce the SEP LET flux.

Figure 33: The integral Linear Energy Transfer (LET) spectrum of Galactic Cosmic Rays modulated to o $\leq 558 \mathrm{MV}$ for a 1 micron thick Silicon sensitive layer as a function of shield thickness (see text for details).

Figure 34: The Galactic Cosmic Ray LET spectrum for a 1 micron Silicon sensitive layer and Aluminum shield thicknesses 
of $1,50,100,200,400,800$ and 1600 mils. The bottom panel (B) includes Anomalous $\mathrm{He}, \mathrm{N}, \mathrm{O}$ and $\mathrm{Ne}$ while the top panel (A) does not. The effects of the Anomalous species is evident when the shield thickness is only 1 mil thick, and is effectively blocked by a shield only 50 mils thickness.

Figure 35. The LET Fluence spectrum for the four samples of SEP events possible during 1994. The calculations were done for a 1 micron Silicon sensitive layer and Aluminum shields of thickness $50,100,200,400,800$ and 1600 mils. Shown are the "Typical" (A), "Heavy Ion Rich" (B), "Heavy Ion Poor (C), and "Large Event" (D) samples 80

Figure 36: The LET fluence spectrum calculated for the Lunar survey period of the Clementine mission $(2 / 21 / 94-5 / 3 / 94)$ is shown for a 1 micron Silicon sensitive layer and shields of 50, 100, 200, 400, 800 and 1600 mils of Aluminum. The particle flux used in this calculation includes Galactic Cosmic Rays and Anomalous species with a solar modulation level of $\phi=588 \mathrm{MV}$ as well as the "Typical" SEP sample fluence appropriately scaled for time period.

Figure.37: Similar to Figure 36 except calculated for the Clementine spacecraft Geographos encounter time period $(5 / 27 / 94$ $8 / 31 / 94)$. The lower level of solar modulation $(\phi=588$ MV) causes a slight increase in fluence for a given LET as compared to the Lunar survey time period.

Figure 38. The LET for the "Typical" SEP sample (top) and Galactic Cosmic Rays with Anomalous species (bottom) calculated for the Interstage Adapter and including the effects of geomagnetic transmission. There is no substantial difference between these plots and those calculated using interplanetary spectra

Figure 39: The LET fluence spectrum calculated for the Interstage Adapter for the time period from 2/1/94 to $10 / 1 / 94$ is shown for a 1 micron Silicon sensitive layer and Aluminum shields of thickness 50, i00, 200,400,800, and 1600 mils. The particle flux used in this calculation includes Galactic Cosmic Rays, Aromalous species and the "Typical" SEP sample fluence scaled for the time period. The level of solar modulation used was $\phi-570$ MV. 


\section{LIST OF TABLES}

Table 1: Characteristics of the GCR Interstellar Transport

Calculation

Table 2: $\quad$ SEP Events During the CRRES Mission ................................................40

Table 3: $\quad{ }^{4} \mathrm{He}$ Spectra and Fluences.....................................................................42

Table 4: $\quad$ Proton Spectra and SEP Model Parameters............................................43

Table 5: $\quad$ Predicted Values of $\phi$ During 1994........................................................61

Table 6: $\quad$ LET Fluence Spectra for Lunar Survey Period ........................................8

Table 7: $\quad$ LET Fluence Spectra for Geographos Period............................................84

Table 8: $\quad$ LET Fluence Spectra for Interstage Adapter..............................................88 


\section{INTRODUCTION}

Lawrence Livermore National Laboratory Subcontract B235271 was established April 1, 1993 at Louisiana State University (LSU) to support studies of the anticipated radiation environment to be encountered by the CLEMENTINE I spacecraft and transstage. With BMDO (formerly SDIO) and NASA, LLNL is involved in the Clementine mission to space test new technology sensors, and their support systems, that have been developed for monitoring purposes. The space radiation environment, particularly the heavy ion component, presents one of the most stringent challenges to system design, particularly with regard to micro-electronic circuitry.

For electronic components located within a spacecraft, there is a large radiation dose (depending upon the actual orbit) contributed by protons and electrons plus radiation effects due to the heavy ion component of galactic cosmic rays (GCR), anomalous component (AC), and solar energetic particles (SEP). This heavy ion component is particularly important because of the ability of a single heavy nucleus to affect ("upset") microelectronic circuitry (e.g. Binder et al., 1975) particularly low power memory chips (Kolasinski, et al., 1979). Even though the relative abundance of heavy ions is small, compared to protons, the heavy ion efficiency is high since the effects are caused directly by the passage of the particle through the device.

It might, at first, seem best to employ the measurements from the particle sensors on CLEMENTINE I directly to determine the radiation environment. This is not practical for several reasons: (1) the CLEMENTINE I spacecraft sensors can directly measure only electrons and protons at low energies missing much of the integral flux because they do not measure all of the highest energy particles or any of the heavy ion component; (2) the trans-stage carries no experiments which can directly measure the particle flux; and (3) a majority of the CLEMENTINE radiation sensors provide integral measurements which can not distinguish between particle charge and energy making it difficult to extrapolate these measurements to arbitrary device configurations.

In order to describe, quantitatively, the heavy ion environment in near Earth space, it is necessary to model the GCR and SEP components separately. Scientific research in cosmic ray physics over the past 30 years has established, accurately, relative abundances and energy spectra for most of the elements. A galactic cosmic ray model can take full advantage of this accumulated knowledge and use contemporary measurements most efficiently to accurately define the cosmic ray interisities over the energy ranges where temporal variations are large and not easily predicted in detail. A description of GCR based upon the well-established physical principles of interstellar propagation and solar modulation that control and determine the time- and energydependent GCR intensity at earth is possible. Such a model would incorporate observations obtained over the past two decades of space research. For the SEP 
component, it is necessary to define the occurrence of the flares, the peak flux, total fluence, composition characteristics, and the form of the energy spectrum. We have developed such models, with reference to the data from the CRRES/SPACERAD mission.

The current effort at LSU provides LLNL with estimates of the radiation environment in the vicinity of Earth during 1994 from the CHIME model recently developed for the CRRES/SPACERAD mission (Chenette et al., 1994). This model uses current physical theory of energetic particle interstellar. transport and solar modulation to predict galactic cosmic ray (GCR) and anomalous component (AC) heavy ion spectra for a particular time period, incorporates the characteristics of most Solar Energetic Particle (SEP) events seen by the CRRES/SPACERAD mission, calculates the geomagnetic transmission of these particles for particular orbits and provides a mechanism for calculating the Linear Energy Transfer (LET) spectra from these inputs. These LET spectra can then be used, with appropriate device models, to determine the expected single event upset rate.

This final report on our effort describes the initial assumptions and context of our study as well as presenting the final results. A general discussion of the GCR, AC and SEP radiation encountered in interplanetary space is provided in section II. This is followed in section III with a description of the CHIME model, its functionality and physical basis. Finally, in section IV particle energy spectra and LET results are presented for both the CLEMENTINE I spacecraft and the trans-stage environment.

\section{THE ENERGETIC PARTICLES}

The heavy ion radiation environment encountered in near-Earth space consists of four major components: (a) galactic cosmic rays (GCR), (b) anomalous component (AC), (c) solar energetic particles (SEP), and (d) trapped magnetospheric particles. The first three of these arrive at the Earth from outside our immediate geospace environment and are termed "interplanetary" while the last resides within the Earth's magnetosphere, and is termed a "local" component. The interplanetary particles encompass a wide range in both energy $(E>500 \mathrm{keV})$ and charge $(1 \leq Z \leq 92)$. Some of these particles penetrate the Earth's magnetic field and form part of the radiation environment in which spacecraft must operate.

In this investigation only the "interplanetary" components for the CLEMENTINE I spacecraft and their transmission to the transstage orbit were studied. The general characteristics of this interplanetary component are described below. 


\section{A. Galactic Cosmic Rays}

For the past several decades the composition and energy spectrum of Galactic Cosmic Ray (GCR) nuclei have been measured by a number of satellite experiments, and there is a wealth of previous measurements which can be used as a baseline. It is now known, for example, that the GCR are an extra solar system matter sample which contain all naturally occurring elements. The relative elemental composition of the GCR is shown in Figure 1 for $\mathrm{H}$ to $\mathrm{Ni}$ as the filled circles and is compared to that of "solar system" material as the.open circles. The composition of most "primary" species (C, O, Ne, Mg, Si, S, Ar, Fe, $\mathrm{Ni})$ show very good agreement, but some elements which are very rare in "solar system" matter (Li-B, Sc-Mn) are enhanced in the GCR by many orders of magnitude. This is an effect of the propagation of GCR nuclei through interstellar space where heavier nuclei $(\mathrm{C}, \mathrm{O}, \mathrm{Fe})$ interact with interstellar $\mathrm{H}$ and He to fragment and produce lighter, "secondary" species. For elements heavier than $\mathrm{Ni}$ measurements are still rather poor, but general agreement with "solar system" composition is indicated up to $Z \approx 60$ while still heavier species appear to require additional $r$-processed material in the sources.

The measured energy spectra of $\mathrm{GCR} \mathrm{H}, \mathrm{He}, \mathrm{C}$ and Fe are shown in Figure 2. These data are only part of the current GCR baseline database which contains close to 1000 data points from more than 50 literature references and covers the most important elemental ratios and spectra. The data show that at high energy (i.e. $>10 \mathrm{GeV} /$ nucleon) the spectral shape is a power law with an index of about -2.7 . At lower energy $(<2 \mathrm{GeV} /$ nucleon) the spectra turn over due to the rigidity and adiabatic deceleration effects as cosmic rays diffuse inward against the outflowing solar wind from local interstellar space to the orbit of Earth (i.e. solar modulation). This turn-over causes the peak cosmic ray flux to occur at an energy between $400 \mathrm{MeV} /$ nucleon and $600 \mathrm{MeV} /$ nucleon. The exception to this are species which have an additional Anomalous Component (see section II.B) which cause the energy spectrum to flatten or turn-up for energy below about $100 \mathrm{MeV} /$ nucleon as shown for He in Figure 2.

The GCR flux in the local interstellar space (LIS) is expected to be stable over time scales of millions of years, but due to the influence of solar modulation time variations on the order of weeks can be seen at the orbit of Earth. In Figure 3 the monthly average of measurements from the Climax neutron monitor from 1953 to 1993 are shown. A neutron monitor is sensitive to neutrons produced in the atmosphere by high energy $(\approx 2 \mathrm{GeV})$ cosmic ray protons and clearly shows the periodic variations of the solar cycle. During solar minimum conditions $(\approx 1954,1965,1975$, and 1987$)$ the GCR flux is enhanced increasing the neutron rate observed on the surface of Earth. During solar maximum periods $(=1958,1970,1982$, and 1991$)$ the cosmic ray flux is repressed. The time period between successive maxima or minima is roughly 11 years and a complete solar cycle, including solar magnetic field polarity reversal, covers 


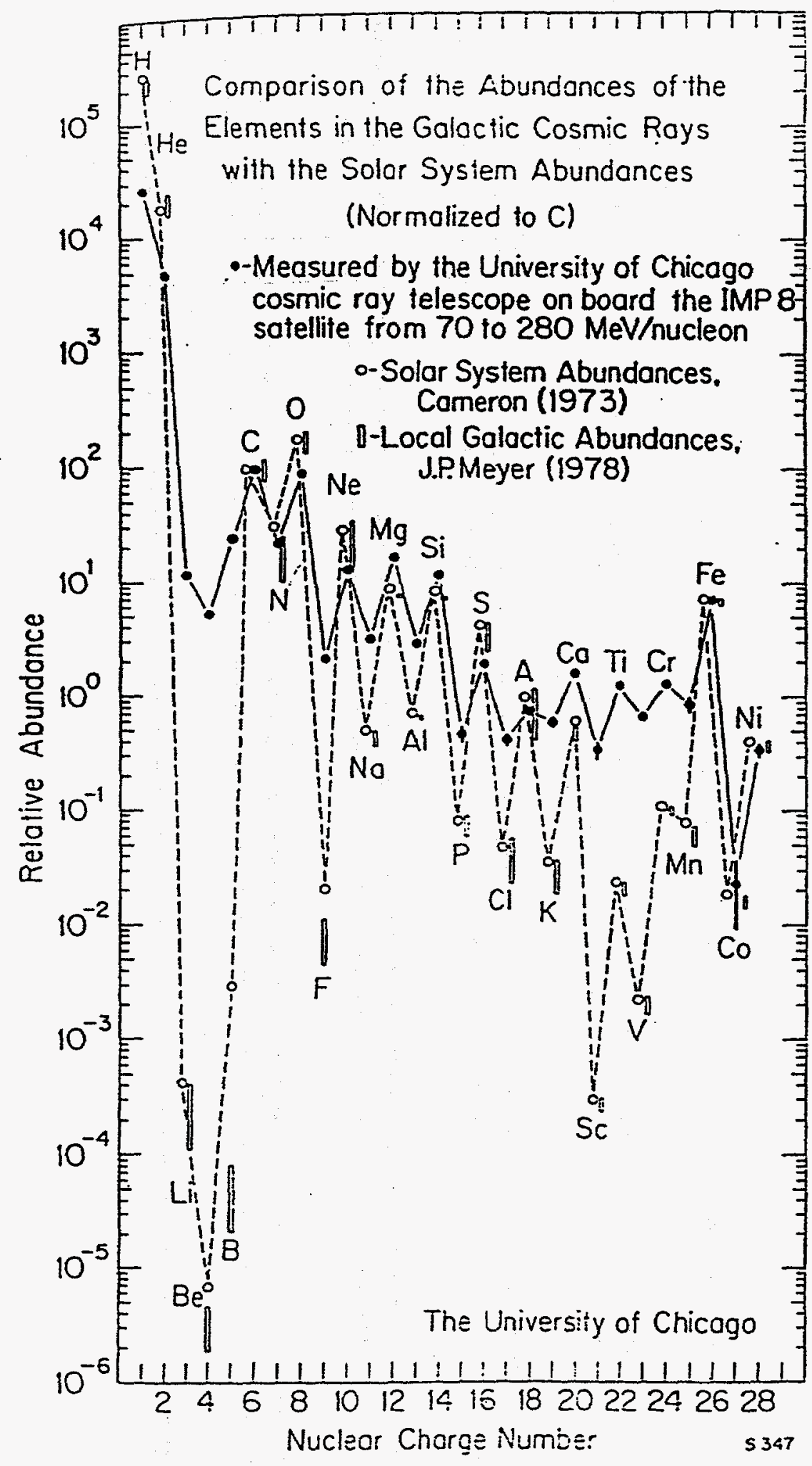

Figure 1: The relative elemental abundances for Solar System material (open circles and dashed line), Local Galactic matter (open bars), and the Cosmic Rays measured near Earth (filled circles and solid lines). 


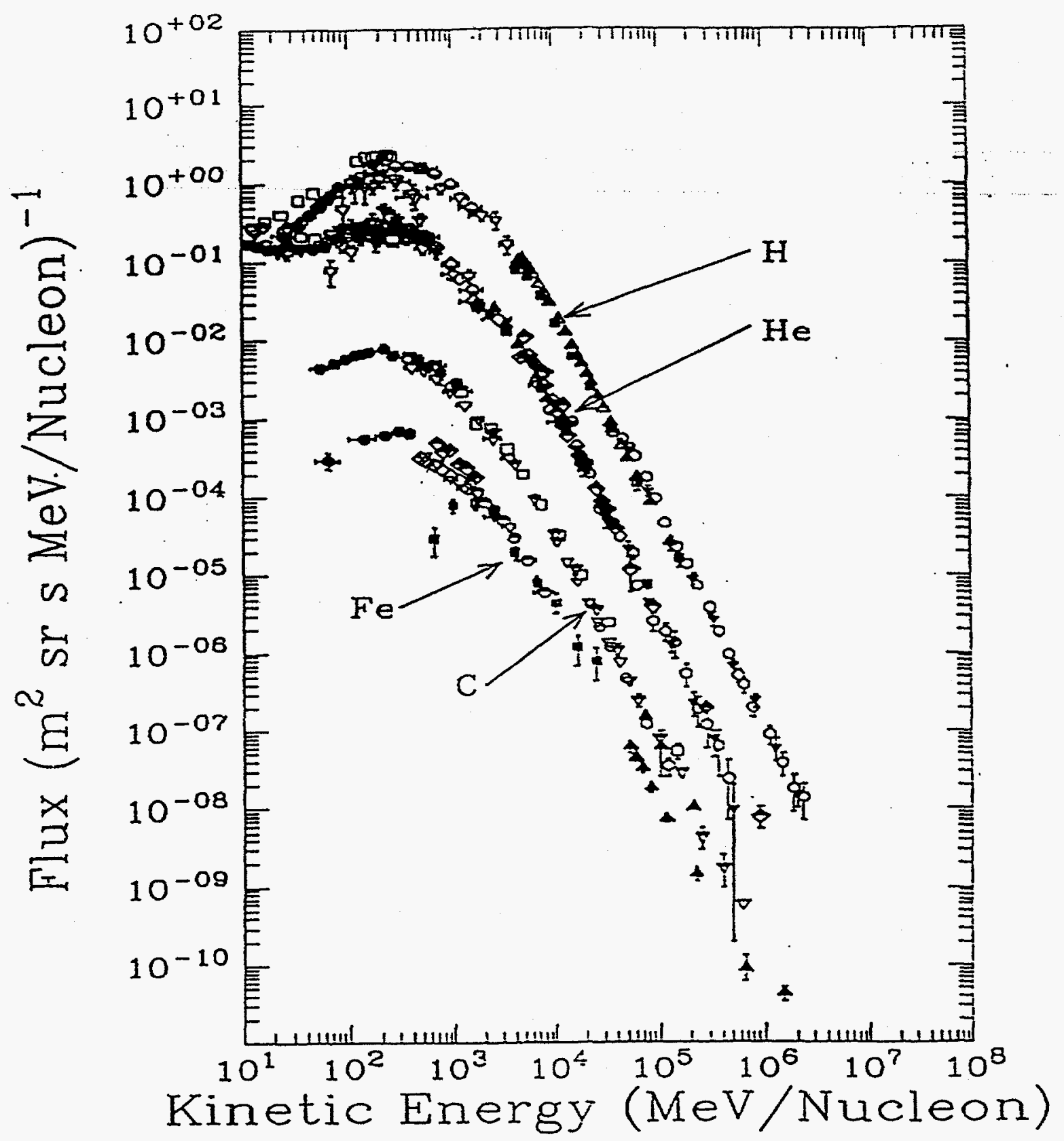

Figure 2: Compiled measurements of the differential energy spectrum for Cosmic Ray $\mathrm{H}, \mathrm{He}, \mathrm{C}$ and $\mathrm{Fe}$ made near $\mathrm{E}$ ath, mostly during solar minimum conditions. At high energies, the spectral shape is a power law with an index of about -2.7. For energies below $-2000 \mathrm{MeV} /$ nucleon solar modulation causes the spectra to turn over giving the peak cosmic ray flux at $400-600 \mathrm{MeV} /$ nucieon. The flattening in the He spectrum below $100 \mathrm{MeV} /$ nucleon is due to the addition of the Anomalous Component. 
about 22 years. Note that during 1992 and 1993 the neutron monitor rate began a recovery to solar minimum conditions and this trend is expected to continue through 1994. Further if the 22 year cycle indicated in Figure 3 is continued, then we may expect an extended solar minimum period similar to that during $1973-1979$.

The solar cycle variation is still not understood in the detail necessary to reliably predict future trends. However, it is clear that a reasonable model of the cycle is necessary to accurately predict the GCR flux, and subsequent LET spectra and SEU rates, for a particular period of time. An empirical model, which is based upon in-situ measurements and a study of the long term solar cycle variation, is used in this investigation and is discussed in the next section. Such a model can be updated as new measurements become available.

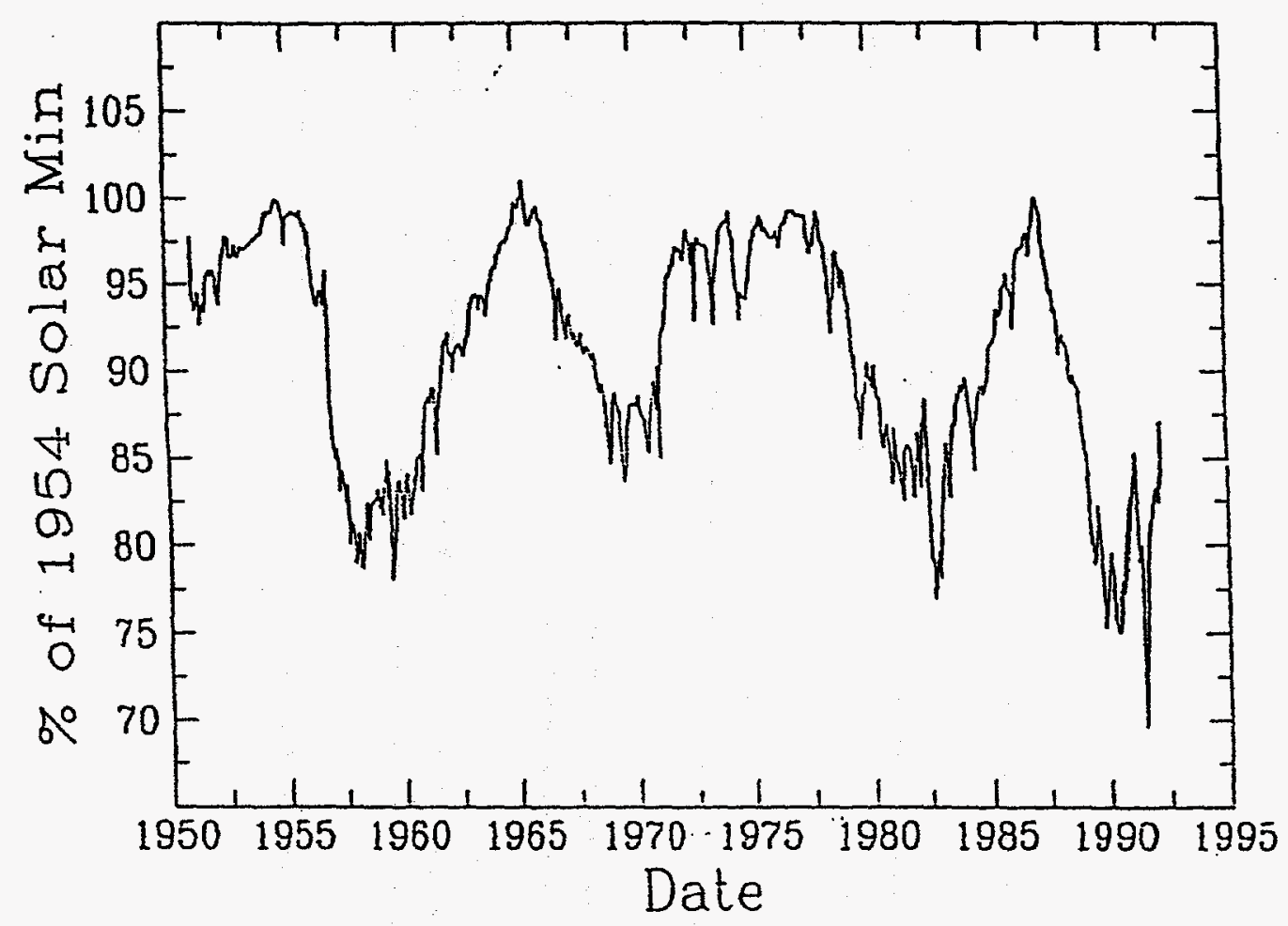

Figure 3: Monthly averaged Climax neutron monitor measurements from 1953 to 1993. During solar minimum conditions the cosmic ray flux is enhanced while during solar maximum conditions the cosmic ray flux is repressed. Note that the last solar maximum (1989-1992) was the most intense maximum observed since 1953.

\section{B. Aromalous Component}

An anomalous component to the observed cosmic ray spectrum has been reported for the elements helium, carbon, nitrogen, oxygen, neon, and argon. All six of these elements have high first ionization potentials ard are present in 
the interstellar medium as neutral atomic matter. Anomalous component cosmic rays are widely believed to originate as neutral atoms in interstellar space, which drift into the inner solar system and are subsequently photoionized by solar UV radiation and swept back outward by the solar wind. Acceleration at the shock boundary of the heliosphere produces a local "interstellar" spectrum, which is then modulated in the heliosphere as the energetic particles move back toward Earth. The energies consistent with acceleration at the heliospheric shock boundary are low, and the $\mathrm{AC}$ local interstellar spectra are consequently very steep, essentially vanishing at energies above about $1 \mathrm{GeV} /$ nucleon.

The characteristics of the $A C$ as illustrated by measurements made at widely spaced locations in the heliosphere are shown in the six panels of Figure 4 which are taken from the recent review of low-energy cosmic rays by Biswas et al. (1993). At energies above about $100 \mathrm{MeV} /$ nucleon the GCR always dominate the flux, but below this energy species such as $\mathrm{He}$ (panel b), with relatively high first ionization potential show a dramatic increase in flux due to the anomalous component. In some cases, the AC flux exceeds the GCR maximum level by several orders of magnitude. In addition, measurements by spacecraft in the outer reaches of the heliosphere show that the AC has an increased presence. Conversely, species with low first ionization potential ( $\mathrm{H}$ in panel a) show a decrease in flux with decreasing energy indicating little or no contribution from the anomalous component.
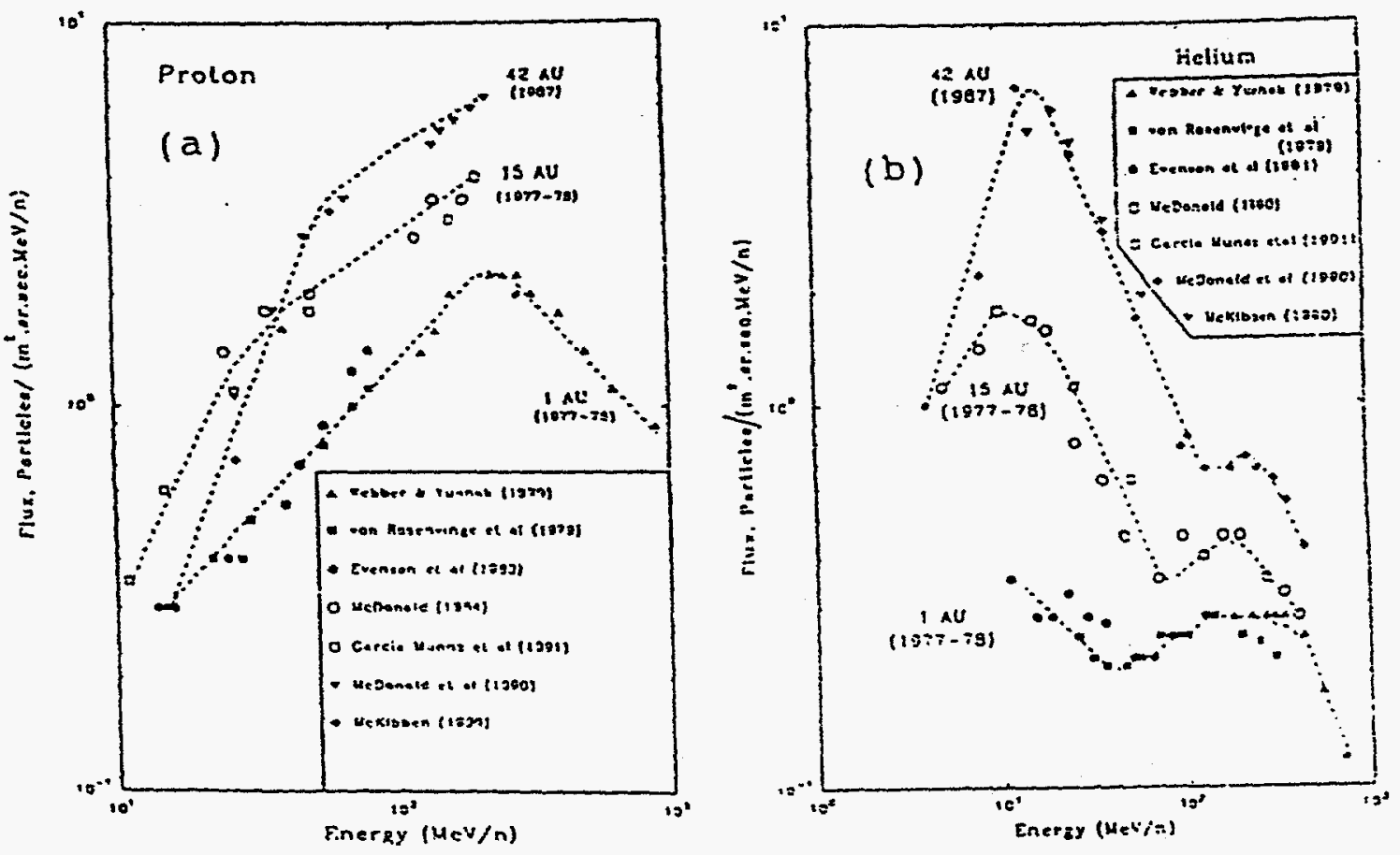

Figure 4a,b: Low energy cosmic ray measurements of (a) Hydrogen and (b) Helium for solar minimum conditions. 

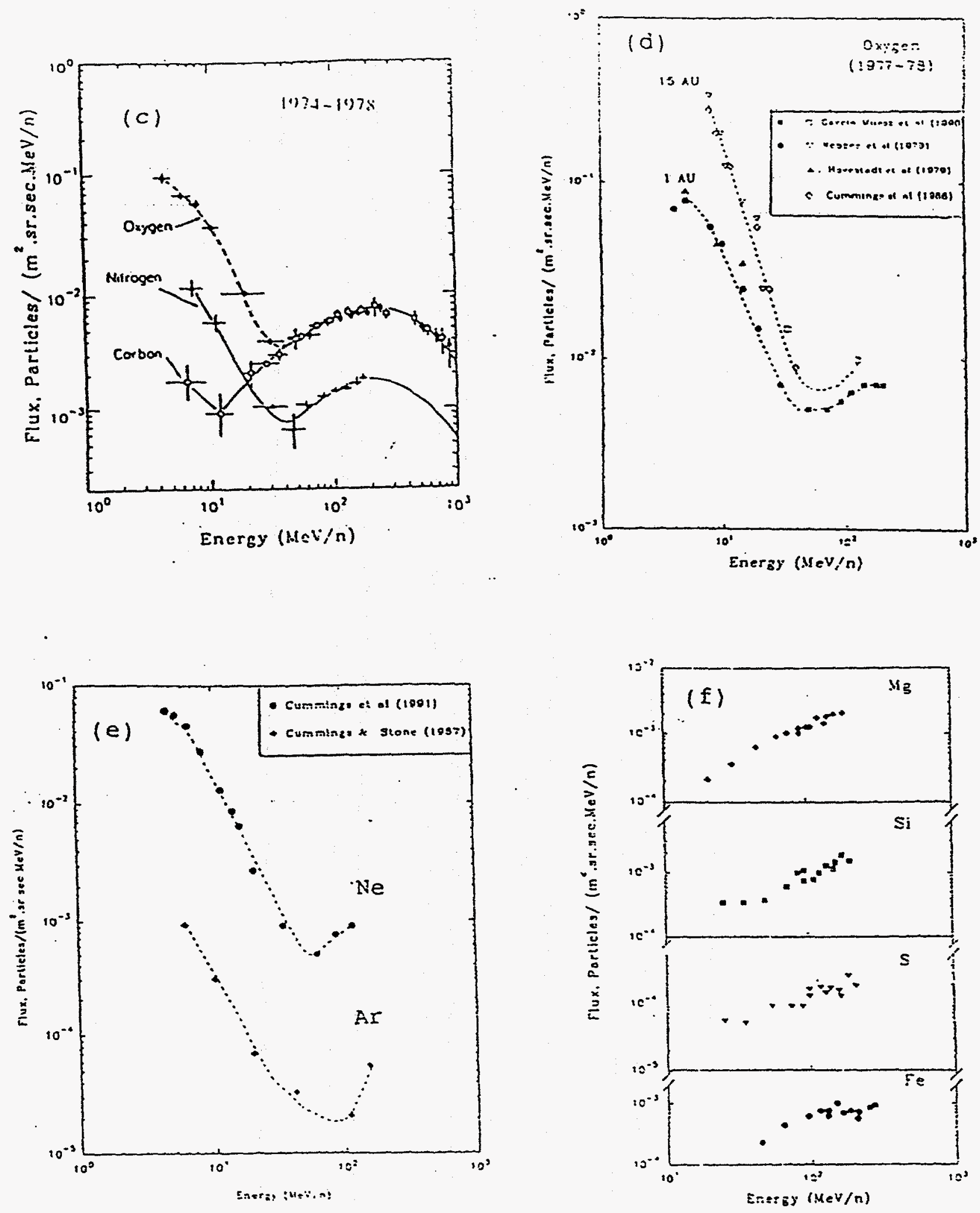

Figure 4c-f: Low energy cosmic ray measurements at solar minimum, and at various locations in the heliosphere, for: (c) $C, N$ and $O$ at 1 A.U., (d) $O$ at 1 and 15 A.U. (e) $\mathrm{Ne}$ and $\mathrm{Ar}$ at 1 A.U., and (f) $\mathrm{Mg}$, Si, S and $\mathrm{Fe}$. 
For heavier elements, there are anomalous components for $\mathrm{O}$ and $\mathrm{N}$ (Figure $4 \mathrm{c}, \mathrm{d}$ ) and for $\mathrm{Ne}$ and $\mathrm{Ar}$ (figure 4 e). The other heavy elements, e.g. $\mathrm{Mg}$, $\mathrm{Si}, \mathrm{S}$ and $\mathrm{Fe}$ in figure $4 \mathrm{f}$ show no low energy enhancement, i.e. no anomalous component. The element $C$ (figure $4 \mathrm{c}$ ) shows some evidence for a low energy turn-up but this is orders of magnitude less significant than the oxygen, even though $\mathrm{C}$ and $\mathrm{O}$ are about equally abundant above $100 \mathrm{MeV} /$ nucleon in the GCR component.

The low energy of the $A C$ cosmic rays accounts for their near absence from measured cosmic ray fluxes during periods of high solar modulation. In fact the AC level tracks the level of solar modulation as shown in Figure 5 where low energy ( $8-27 \mathrm{MeV} /$ nucleon) anomalous Oxygen measured near Earth (data points) from 1972 to 1988 is compared with the scaled value of the Mt. Washington neutron monitor rate (solid line). Little AC Oxygen is seen during the solar maximum of 1980 to 1984 , but is clearly present during solar minimum. With the return to solar minimum during 1994, it is expected that the heavy ion anomalous component flux will also be present.

Since the $A C$ species are ionized by solar $U V$, one expects the $A C$ cosmic rays to be singly charged. Relative to an equivalent velocity $G C R$, the $A C$ is, therefore, much more rigid $(R=p / Z)$ and consequently has a much higher transmission through the Earth's magnetic field. Thus, these low energy heavy ions could make a significant contribution to the SEU rate in unshielded electronic components even deep within the geomagnetosphere.

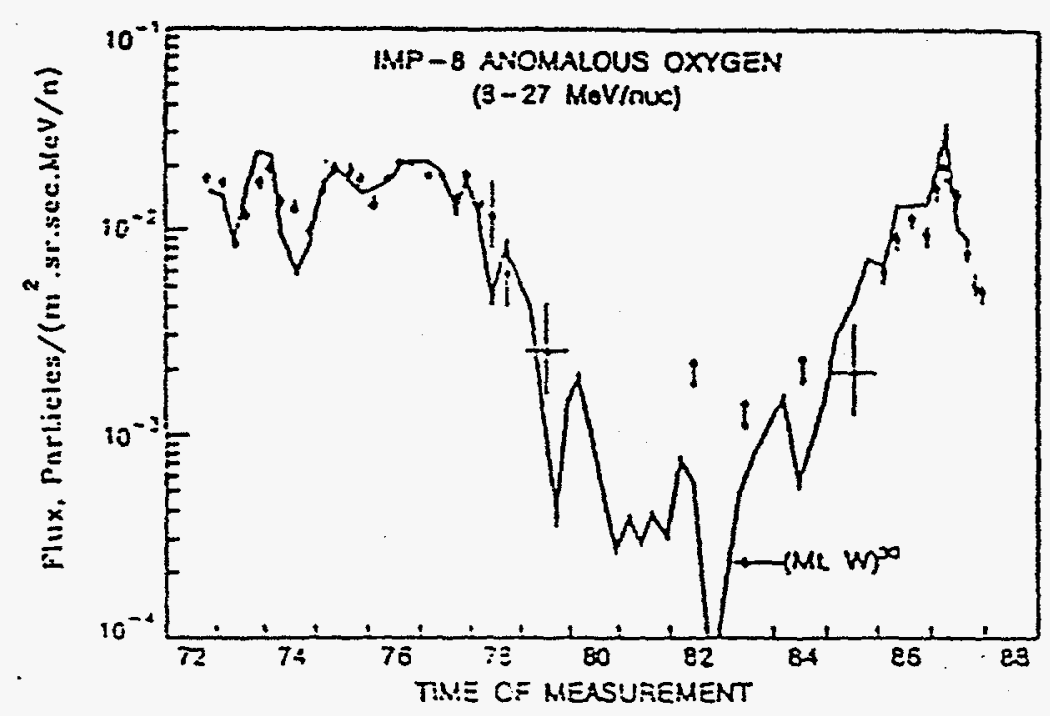

Figure 5: Low energy (8-27 MeV/nucleon) Anomalous Oxygen measured near Earth (data points) from 1972 to 1988 compared with the scaled value of the Mt. Washington neutron monitor rate (solid line). 


\section{Solar Energetic Particles}

Anytime during the solar cycle, though more often during periods of solar maximum, solar flare eruptions can generate conditions which accelerate ions in regions connected to the interplanetary magnetic field, allowing energetic particles to escape the vicinity of the sun and, subsequently, to be observed near Earth as Solar Energetic Particle (SEP) events. While the GCR are a continuous, highly penetrating background radiation, SEP events are usually of short duration but with, at times, very intense fiuxes and, occasionally, significant amounts of heavy ions. The variations of SEP energy spectra, heavy ion content, duration and occurrence make it very difficult to predict trends and to reliably determine the contribution of SEP's to a LET spectrum for a given time period in the future. The current model used in this study incorporates observations made by the CRRES/SPACERAD experiments during the last solar maximum period (1991), supplemental observations from the GOES and IMP-8 satellites, both essentially in interplanetary space, and a proton fluence model by Feynman et al. (1993).

The energy spectrum of a SEP can vary across a broad range of forms, but, in many cases, a power law fit can represent the SEP energy spectrum very well (Guzik, 1988) in the high energy region. For example, Figure 6 shows the energy spectrum of protons and Helium for a typical event (panel A) and a very large event (panel B) which occurred during the solar maximum period of 1991 . In this figure the proton measurements (solid circles) are from the GOES satellite, the Helium data (open squares) were measured by the ONR-604 instrument on the CRRES satellite (Chen et al., 1994), and the solid curves are power law fits to these data. Notice that in both cases the Helium spectrum is steeper than the proton spectrum, and of the 26 events observed by CRRES and GOES during 1991, this is generally the case. The SEP events usually last only a short period of time (days), but can greatly exceed the GCR flux up to energies of several hundred $\mathrm{MeV} /$ nucleon. For comparison with Figure 6, the peak of the GCR Hydrogen flux from Figure 2 would be $2 \times 10^{-4}\left(\mathrm{~cm}^{2} \mathrm{sr} \mathrm{s} \mathrm{MeV}\right)^{-1}$ at about $400 \mathrm{MeV}$, while that for Helium would be $3 \times 10^{-5}\left(\mathrm{~cm}^{2} \mathrm{sr} \mathrm{s} \mathrm{MeV/nucleon}\right)^{-1}$ at about 300 $\mathrm{MeV} /$ nucleon.

Selective heating by wave-particle interactions in the flare plasma can produce significant enhancements of heavy ions in particular SEP events (Reames, Meyer, and von Rosenvinge, 1994 and reviews by Ramaty et al., 1980; Kocharov \& Kocharov, 1984; Melrose, 1990). An example is shown in. Figure 7 from the work of Dietrich and Simpson (1978) for the 24 Sept. 1977 flare. Here the relative enhancement of SEP even- $Z$ elemental abundances over "solar system" composition versus the charge of the element measured is shown. For this SEP event, Fe was enhanced by a factor of 20 . Referring back to Figure 1 , this enhancement means that $\mathrm{Fe}$ would be as abundant as $\mathrm{O}$ in this energetic particle population. Thus, the LET spectrum for such "Heavy Ion Rich" events would show an enhanced flux at high LET. Conversely, the steep energy spectrum of 

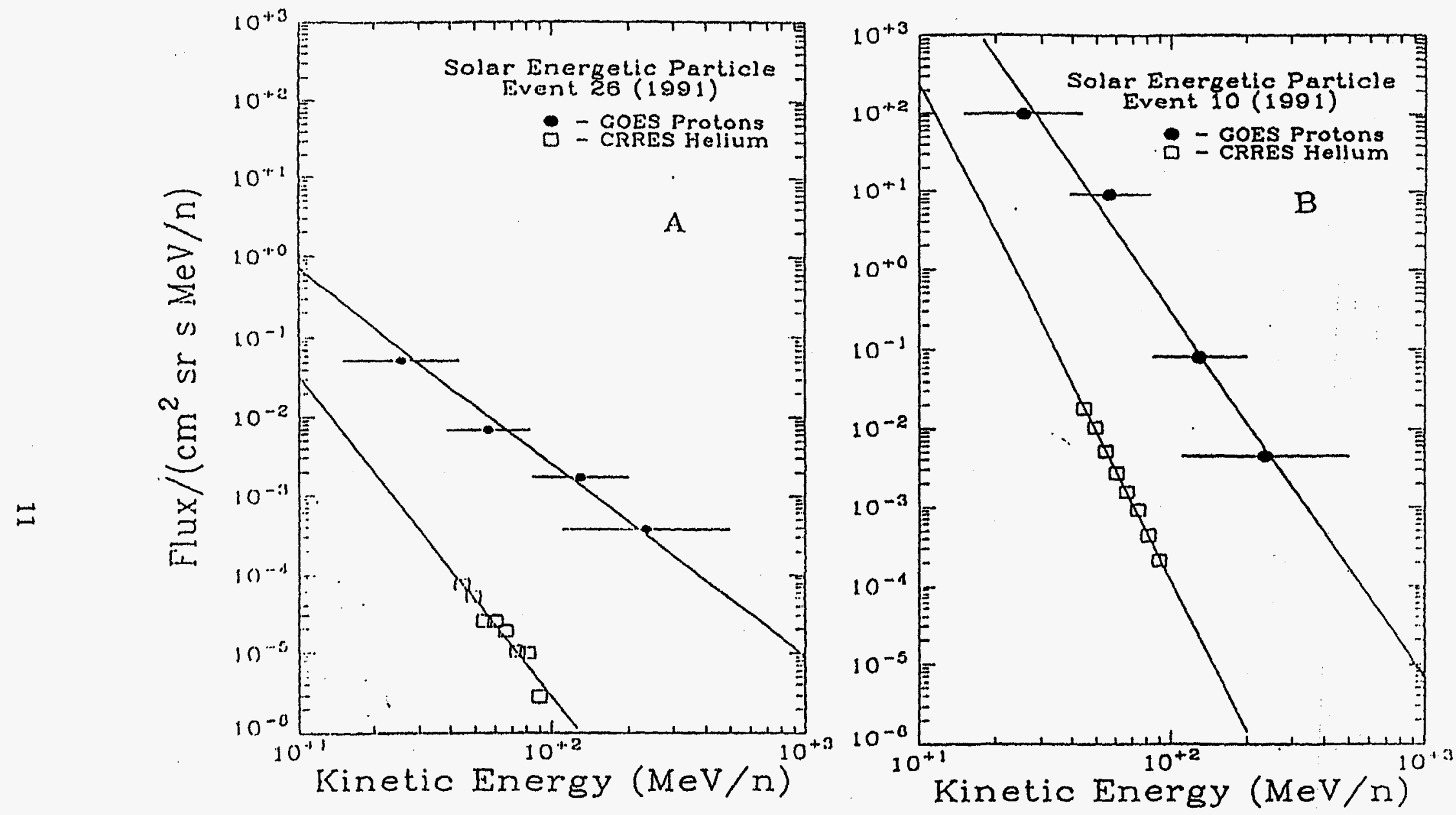

Figure 6: Proton and Helium flux measurements for an "average" flare (Event 26, panel A) and the largest flare (Event 10, panel B) seen during 1991. The proton measurements (filled circles) were obtained from GOES and the Helium flux points (open squares) are from the ONR-604 instrument on-board the CRRES spacecraft. The curves are power law fits to the data, and the parameters for these spectra are given in Tables 2-4. 


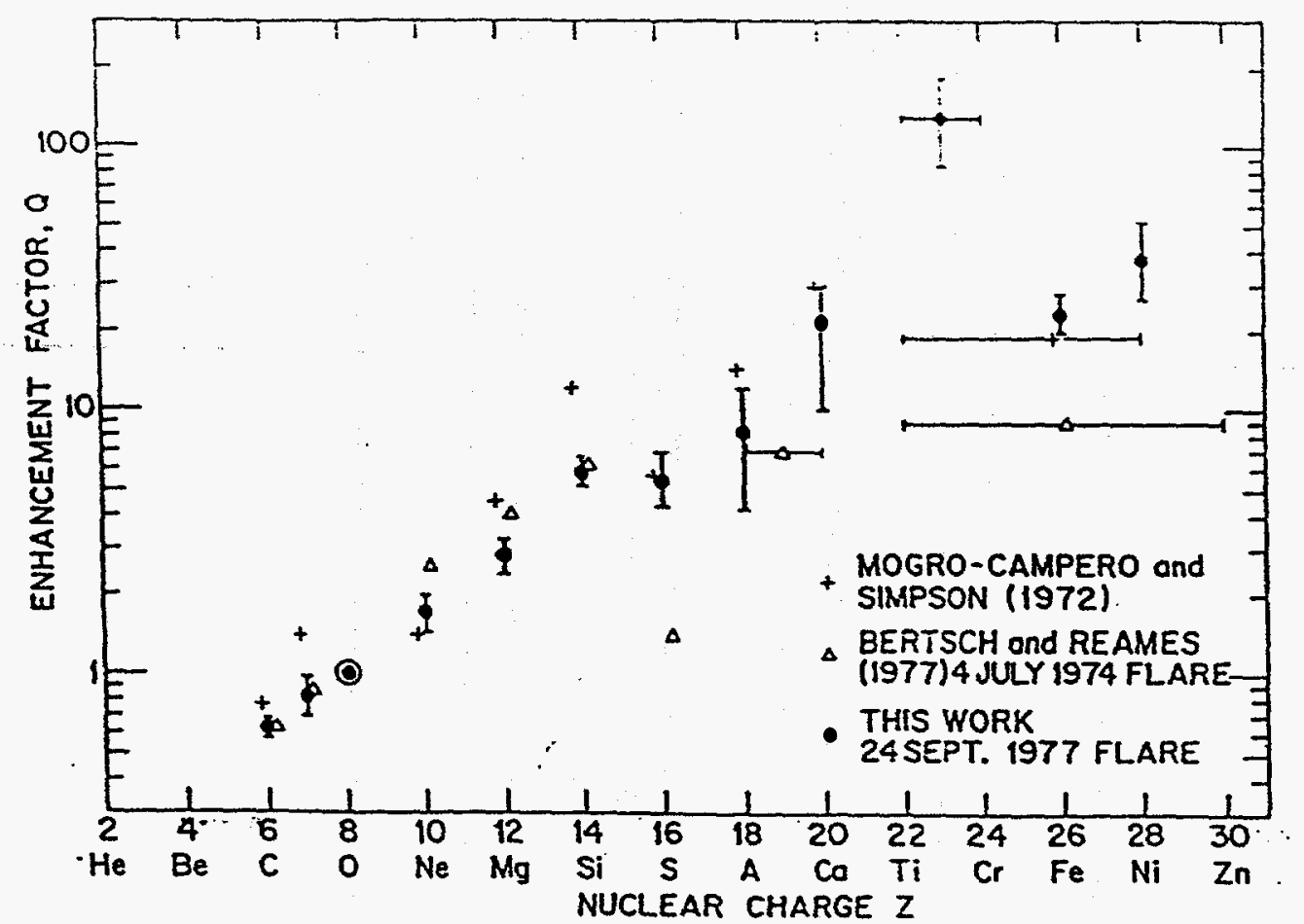

Figure 7: The relative enhancement of SEP even- $Z$ elemental abundances over "solar system" composition versus the charge of the element. The: enhancements are normalized to Oxygen $=1$.

SEP events means that this high LET component can be significantly reduced by relatively light shielding, as discussed in subsequent sections.

The number of SEP events follows, as one might expect, the solar cycle. In Figure 8 the mean monthly sunspot number from the Solar-Geophysical Data reports over the time period 1950 to 1993 is plotted as the solid line. The solar cycle is clearly seen with maximum peaks at 1958, 1968, 1979 and 1989. Also shown in the figure, as the dashed line, is the yearly number of SEP events, for protons $>10 \mathrm{MeV}$, observed by instruments on the GOES and IMP-8 spacecraft from 1976 to 1993. Due to instrument detection efficiencies, the dashed line does not represent the total number of SEP events occurring during this time period. It does, however, approximate the number of events encountered by a particular interplanetary spacecraft.

The observed number of SEP events follows the sunspot number, though it does appear that the peak event number lags the peak sunspot number by a couple of years. The total proton fluence from SEP events as a function of time is shown in Figure 9 for the years 1956 to 1993 . Prior to 1976 the fluence is determined from the Feynman et al. (1993) model, and after 1976 it is determined from the GOES/IMP-8 observations. Notice that the total fluence can remain high (or even increase) for several years following the sunspot 


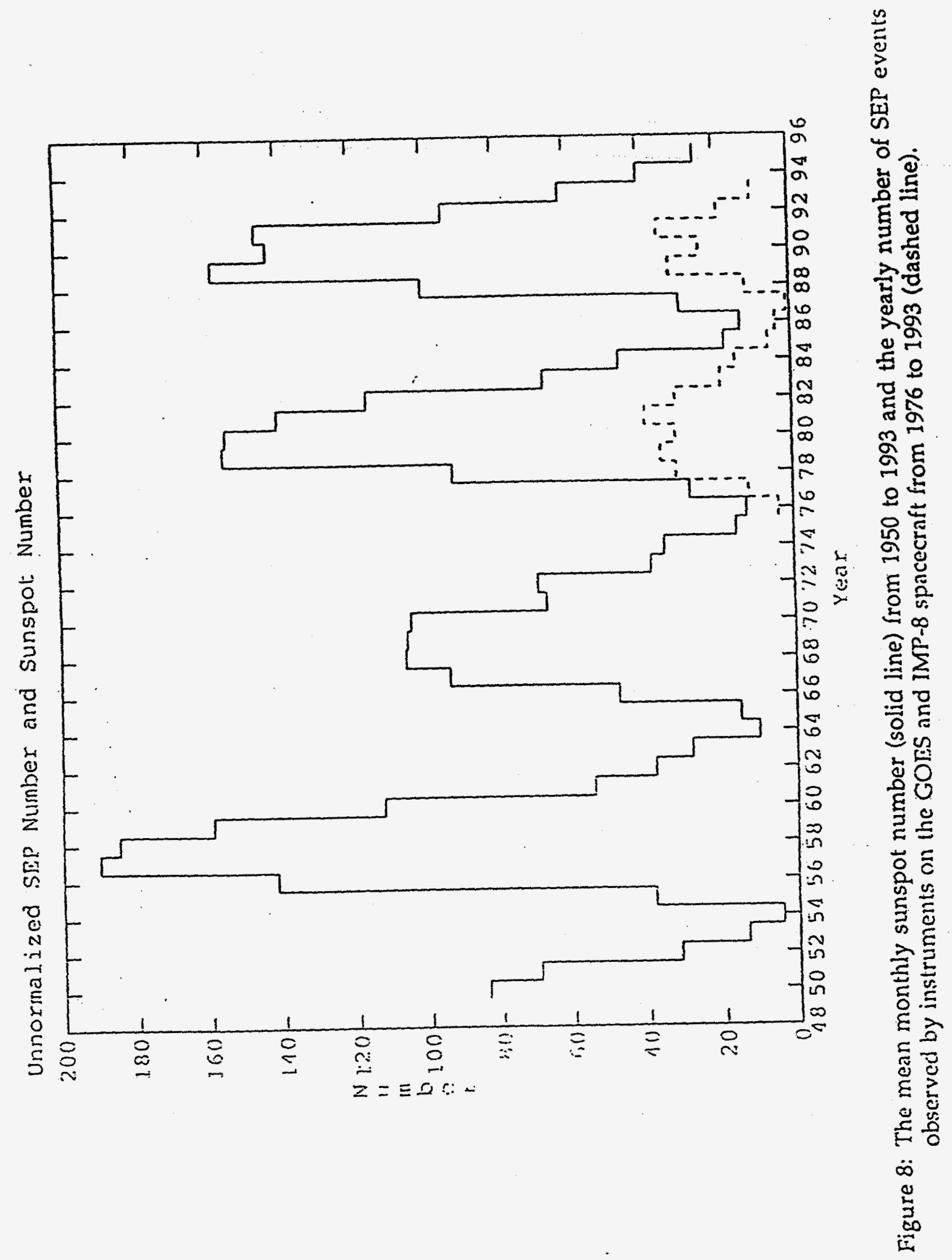




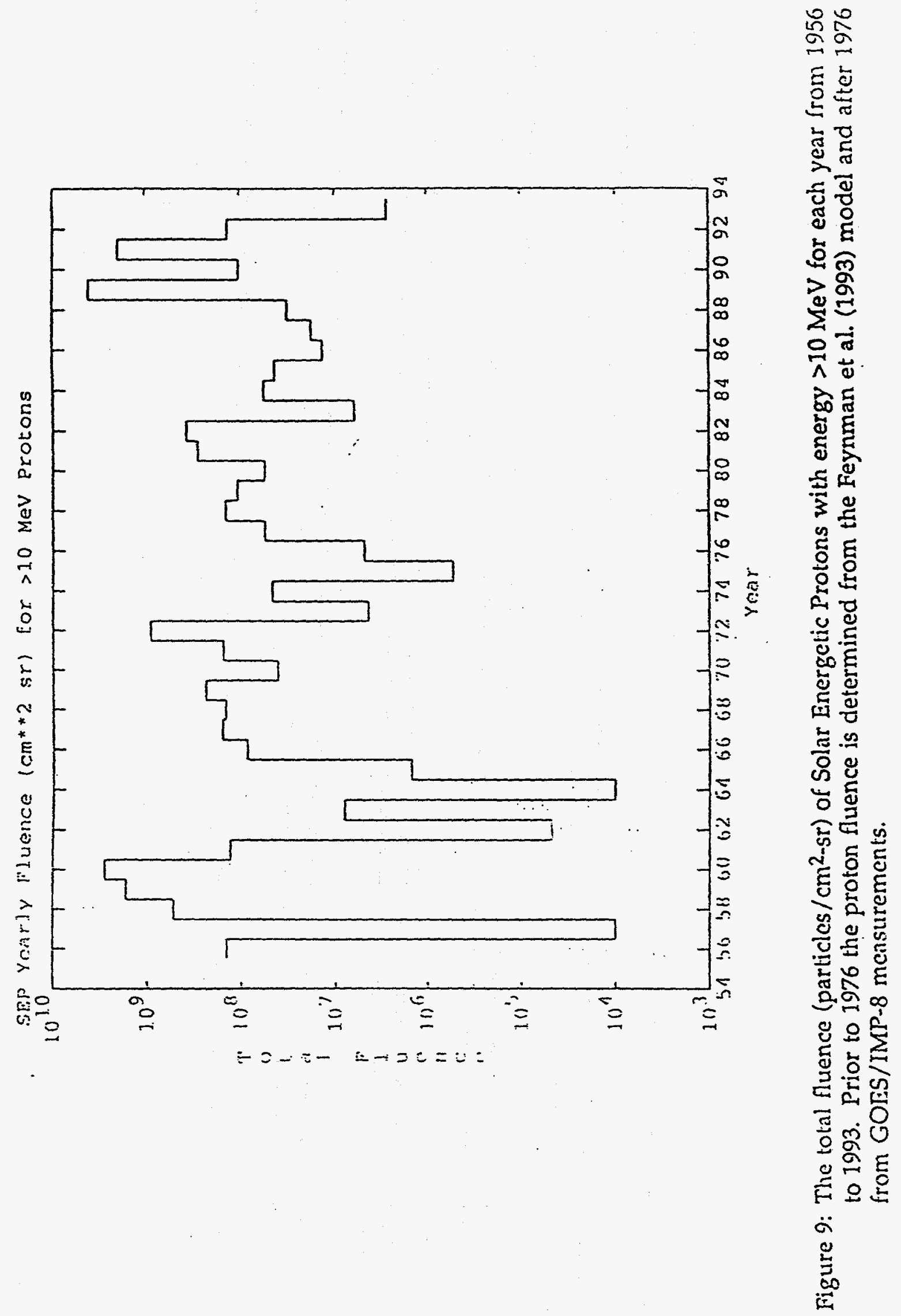


maximum. Such trends need to be taken into account when attempting to predict the number and intensities of SEP events dir-ing the CLEMENTINE I mission.

\section{THE CHIME ENERGETIC PARTICLE MODEL}

\section{A. Scope and Functionality}

The major components of the CRRES/SPACERAD Heavy Ion Model of the Environment (CHIME) used in this study are indicated in Figure 10. The model covers the energy range from $10 \mathrm{MeV} /$ nudeon to $60 \mathrm{GeV} /$ nucleon for all stable elements, and includes the known major sources of heavy ions in the nearEarth interplanetary medium over this energy range; galactic cosmic rays, the anomalous component, and SEP heavy ions. This new model addresses the following major objectives and improvements to the existing state-of-the-art:

1. provides an accurate representation of the heavy ion flux environment and its temporal variations near Earth as measured over the past two solar activity cycles,

2. provides a physically - based method to extrapolate this environment into the future which also can be adapted based on future knowledge of the solar activity cycle,

3. improves the calculation of the geomagnetic cutoff and shielding effects, and

4. packages these elements together in a form which is easy to use by the space radiation effects community.

A new and distinguishing feature of the CHIME model is that the fundamental particle energy spectra used in the calculation are based upon current astrophysical knowledge of cosmic ray origin and transport through interstellar space and the heliosphere rather than on a collection of empirical fits. This knowledge, in turn, is based upon decades of research and over thirty years of direct satellite measurements. The advantage of this approach is that it provides a complete representation of the radiation environment that is in agreement with available measurements and which provides details that are the consequence of the theory but which may not yet be accurately measured.

A primary input to the CHIME model is the Local Interstellar Spectrum (LIS) of GCR which is obtained from a physical model of cosmic ray interstellar propagation using current astrophysical knowledge of the cosmic ray source. These LIS are "modulated" using, again, a physical model of the energetic particle transport through the heliosphere to obtain predicted spectra at the orbit of the Earth. By comparing measurements with the predictions, we were able to 


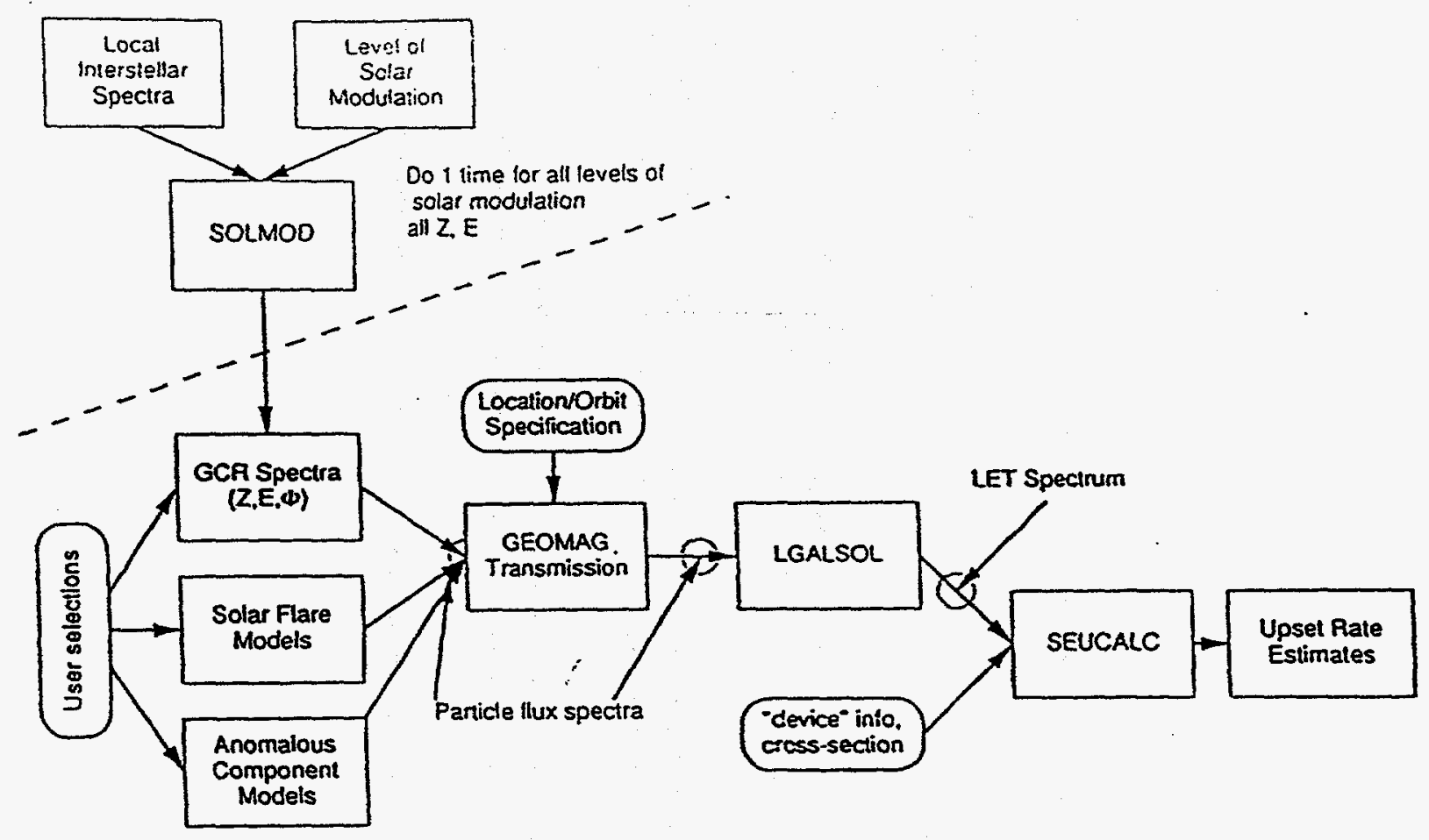

\section{CRRES/SPACERAD Cosmic Ray Model Organization}

Figure 10: Flow chart of the Cosmic Ray Model developed for the CRRES/ SPACERAD mission and used in evaluating the CLEMENTINE radiation environment.

"calibrate" the GCR and AC LIS along with the level of solar modulation ( $\phi)$ as a function of time. A database of GCR and AC spectra as a function of $Z, E$, and $\phi$, and SEP characteristics, then, form the base of the CHIME model. The model user can select from this database for a particular time period and solar flare conditions to form the predicted interplanetary radiation environment. The particle flux spectra can then be modified by a geomagnetic field transmission calculation to obtain spectra for a particular orbit. Either the spectra at the orbit or the interplanetary spectra can be fed directly into LGALSOL which calculates the Linear Energy Transfer (LET) spectrum. Finally, the LET spectrum, along with an appropriate device description, can be used to predict single event upset rates. This latter component of the CHIME model was not used during the present study.

The original motivation for the CHIME model was to provide the most faithful possible description of the intensities of energetic heavy ions and their variations near Earth (outside the radiation belts), and to describe the specific heavy ion environment during the period of the CRRES mission (Chen et al., $1994 a, b)$ by using the heavy ion flux measurements made by instruments of the CRRES payload (Gussenhoven, Mullen and Sagalyn, 1985). It was quickly 
recognized that the best application of the CRRES payload flux measurements would be to use them to establish the parameters of a suitable time- and energydependent model which accurately describes the effects of solar activity on a fixed source. The knowledge accumulated ove: the past three decades or more of cosmic ray physics, which has very accurately determined the relative abundances and energy spectra of the elements, can be incorporated into the model in this way. Contemporary measurements establish the epoch, which is associated with a specific level of solar activity.

\section{B. The Physical Basis of CHMME}

\section{The Local Interstellar Particle Spectrum}

The primary input to the CHIME model is the local interstellar spectra (LIS) of the energetic particles and these are calculated once, taking into account current knowledge of cosmic ray astrophysics. Figure 11 provides a simplified illustration of the.physical processes used in the LIS calculation. At the GCR source, a mixture of material is accelerated to high energies and begins diffusing

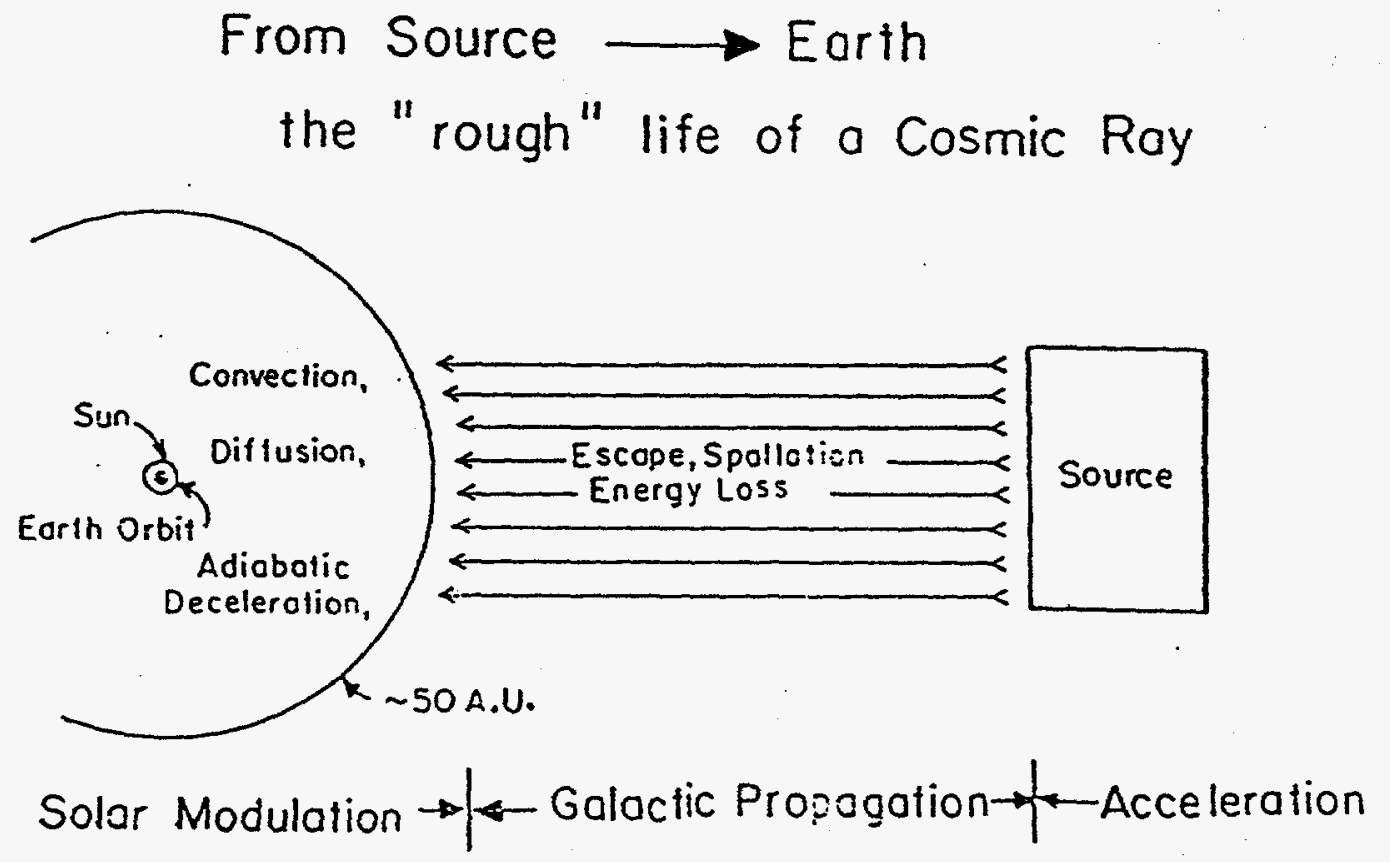

Figure 11: Schematic drawing of the transpo:t of cosmic ray particles from the "source" to the orbit of Earth wh:e:e they are observed.

throughout the galaxy. During this "interst:iar propagation" the high energ: particles can interact with the interstellar mesium and spallate to lighter fragments, radioactive species can decay, anc all species can lose energy or escape from the boundaries of the galaxy. Once near the heliosphere boundary (shown in the figure at -50 A.U.), the GCR and locally accelerated AC particles diffuse inward toward the orbit of Earth encountering convection due to the outflowing 
solar wind and undergoing adiabatic deceleration. A more detailed description of these processes are provided in the following sections along with the LIS derived for the GCR and AC.

\section{a. Galactic Cosmic Ray Interstellar Propagation}

The galactic cosmic ray LIS was calculated using a computer program that is based upon the weighted-slab GCR interstellar propagation technique (Fichtel and Reames, 1968) and which is fully described in Garcia-Munoz et al. (1987). In essence, this technique starts with a set of relative abundances of elements and isotopes at the GCR source, assumes a common source energy spectrum, as well as a particular interstellar medium (ISM) composition and density, and then calculates new abundances and spectra for a series of discrete ISM slabs of matter. The source characteristics used for the slab calculations are provided in Table 1. This calculation includes the effects of production and loss of species via nuclear interaction, radioactive decay ( $\beta^{+}, \beta$-, and $\mathrm{K}$ capture), electron capture and loss, and ionization energy loss. These "slab results" are then integrated over an assumed pathlength distribution (PLD) to obtain the LIS.

One might note at this point that the "Slab Calculations" involve only atomic and nuclear effects which can, in principal, be measured in the laboratory, while the PLD involves the astrophysical details of GCR interstellar propagation and must be either theoretically derived or obtained from the GCR data. Thus, obtaining a good representation of the Galactic Cosmic Rays. is actually a multi-parameter problem, and it is important to use laboratory measurements, GCR data and other astrophysical information to constrain as many of these parameters as possible. In particular, we use a database of cosmic ray spectra and composition ratio measurements obtained from the published literature to provide part of these constraints. An example of this database was shown earlier in Figure 2, which plotted the energy spectra measurements available for the "primary" species $\mathrm{H}, \mathrm{He}, \mathrm{C}$ and $\mathrm{Fe}$. The relative abundance of "primaries" in the measured GCR is dominated by their abundance at the source and is not significantly affected by the "secondary" component generated by the nuclear fragmentation of heavier species. Therefore, primary species reflect. conditions at the cosmic ray source rather than conditions during GCR propagation.

Pure secondary species, which have a negligible source component, are very sensitive to the exact form of the PLD. In fact, by requiririg multiple secondary to primary ratios widely separated in charge to be fit simultaneously, the details of the PLD parameter in the GCR Model can be constrained. Figure 12 shows measurements from the GCR database for the B/C secondary to primary ratio (top) as well as the collection of data for the ratio Sub-Fe/Fe (bottom) where the "Sub-Fe" group includes the elements $\mathrm{Sc}, \mathrm{Ti}, \mathrm{V}, \mathrm{Cr}$ and $\mathrm{Mn}$. Due to the details of the nuclear in teractions which occur during interstellar propagation, these two ratios constrain different portions of the PLD. 
TABLE 1: Characteristics of the GCR Interstellar Transport Calculation

Source Energy Spectrum: $J \propto(P+0.5)^{-2.5}$

Interstellar Material Composition: $93.7 \%$ Hydrogen, $6.3 \%$ Helium

Interstellar Material Density: 0.2 atoms $/ \mathrm{cm}^{3}$

Relative Abundances Used for GCR Source Composition

\begin{tabular}{|c|c|c|c|c|c|}
\hline Isotope & Abundance & Isotope & Abundance & Isotope & Abundance \\
\hline${ }^{1} \mathrm{H}$ & $1.96160 E+09$ & ${ }^{2} \mathrm{H}$ & $0.00000 \mathrm{E}+\infty 0$ & ${ }^{3} \mathrm{He}$ & $0.00000 \mathrm{E}+00$ \\
\hline${ }^{4} \mathrm{He}$ & $1.60800 \mathrm{E}+08$ & $\sigma_{\mathrm{Li}}$ & $0.00000 E+00$ & $7_{\mathrm{Li}}$ & $0.00000 \mathrm{E}+00$ \\
\hline $7_{\mathrm{Be}}$ & $0.00000 E+00$ & ${ }^{9} \mathrm{Be}$ & $0.00000 E \div 00$ & ${ }^{10} \overline{\mathrm{Be}}$ & $0.00000 E+00$ \\
\hline${ }^{10} \mathrm{~B}$ & $0.00000 \mathrm{E}+00$ & $1_{B}$ & $0.00000 \mathrm{E}+00$ & ${ }^{12} \mathrm{C}$ & $4.05570 \mathrm{E}+06$ \\
\hline${ }^{13} \mathrm{C}$ & $4.54240 \mathrm{E}+04$ & ${ }^{14} \mathrm{~N}$ & $1.89240 \mathrm{E}+05$ & $15 \mathrm{~N}$ & $7.59902 E+02$ \\
\hline 160 & $5.11800 E+06$ & 170 & $2.05200 E+03$ & 180 & $1.02600 \mathrm{E}+04$ \\
\hline${ }^{19} \mathrm{~F}$ & $0.00000 \mathrm{E}+00$ & ${ }^{20} \mathrm{Ne}$ & $4.31400 E+05$ & ${ }^{21} \mathrm{Ne}$ & $1.21000 \mathrm{E}+03$ \\
\hline${ }^{22} \mathrm{Ne}$ & $1.72400 \mathrm{E}+05$ & $23 \mathrm{Na}$ & $3.58900 \mathrm{E}+04$ & ${ }^{24} \mathrm{Mg}$ & $8.58350 \mathrm{E}+05$ \\
\hline $25 \mathrm{Mg}$ & $1.09870 \mathrm{E}+05$ & $26 \mathrm{Mg}$ & $1.21890 \mathrm{E}+05$ & $26 \mathrm{Al}$ & $0.00000 \mathrm{E}+00$ \\
\hline${ }^{27} \mathrm{Al}$ & $8.64000 \mathrm{E}+04$ & $28 \mathrm{Si}$ & $9.01000 \mathrm{E}+05$ & ${ }^{29} \mathrm{Si}$ & $5.40000 \mathrm{E}+04$ \\
\hline${ }^{30} \mathrm{Si}$ & $4.50000 \mathrm{E}+04$ & $31 p$ & $8.25000 \mathrm{E}+03$ & $32 \mathrm{~S}$ & $1.19710 E+05$ \\
\hline${ }^{33} \mathrm{~S}$ & $9.57781 \mathrm{E}+02$ & ${ }^{34} \mathrm{~S}$ & $5.31945 E+03$ & $36 s$ & $1.26011 \mathrm{E}+01$ \\
\hline${ }^{35} \mathrm{Cl}$ & $0.00000 E+00$ & ${ }^{36} \mathrm{Cl}$ & $0.00000 \mathrm{E}+00$ & ${ }^{37} \mathrm{Cl}$ & $0.00000 E+00$ \\
\hline${ }^{36} \mathrm{Ar}$ & $1.51374 \mathrm{E}+04$ & ${ }^{37} \mathrm{Ar}$ & $0.00000 \mathrm{E}+00$ & $38 \mathrm{Ar}$ & $2.82659 E+03$ \\
\hline${ }^{40} \mathrm{Ar}$ & $3.59966 \mathrm{E}+01$ & ${ }^{39} \mathrm{~K}$ & $0.00000 E+00$ & $40 \mathrm{~K}$ & $0.00000 \mathrm{E}+00$ \\
\hline${ }^{41} \mathrm{~K}$ & $0.00000 E+00$ & ${ }^{40} \mathrm{Ca}$ & $4.94536 E+04$ & ${ }^{41} \mathrm{Ca}$ & $0.00000 \mathrm{E}+00$ \\
\hline${ }^{42} \mathrm{Ca}$ & $3.26321 E+02$ & ${ }^{43} \mathrm{Ca}$ & $7.39254 \mathrm{E}+01$ & ${ }^{44} \mathrm{Ca}$ & $1.05009 E+03$ \\
\hline $46 \mathrm{Ca}$ & $1.68215 E+00$ & ${ }^{48} \mathrm{Ca}$ & $9: 43535 E+01$ & ${ }^{45} \mathrm{Sc}$ & $2: 93100 \mathrm{E}+01$ \\
\hline${ }^{44} \mathrm{Ti}$ & $0.00000 \mathrm{E}+00$ & ${ }^{46} \mathrm{Ti}$ & $0.00000 E+00$ & ${ }^{47} \mathrm{Ti}$ & $0.00000 \mathrm{E}+00$ \\
\hline${ }^{48} \mathrm{Ti}$ & $0.00000 \mathrm{E}+00$ & ${ }^{49} \mathrm{Ti}$ & $0.00000 E+00$ & $50 \mathrm{Ti}$ & $0.00000 \mathrm{E}+00$ \\
\hline $49 \mathrm{~V}$ & $0.00000 \mathrm{E}+00$ & $50 \mathrm{~V}$ & $4.80400 \mathrm{E}-01$ & $51 \mathrm{~V}$ & $2.39700 \mathrm{E}+02$ \\
\hline${ }^{50} \mathrm{Cr}$ & $2.64920 E+02$ & ${ }^{51} \mathrm{Cr}$ & $0.00000 \mathrm{E}+00$ & ${ }^{52} \mathrm{Cr}$ & $5.15570 \mathrm{E}+03$ \\
\hline${ }^{53} \mathrm{Cr}$ & $5.91500 \mathrm{E}+02$ & ${ }^{54} \mathrm{Cr}$ & $1.47850 E+02$ & ${ }^{53} \mathrm{Mn}$ & $0.00000 E+00$ \\
\hline${ }^{54} \mathrm{Mn}$ & $0.00000 E+00$ & $55 \mathrm{Mn}$ & $0.00000 E+00$ & $54 \mathrm{Fe}$ & $6.26400 E+04$ \\
\hline${ }^{55} \mathrm{Fe}$ & $0.00000 E+00$ & $56 \mathrm{Fe}$ & $9.90000 \mathrm{E} \div 05$ & ${ }^{57} \mathrm{Fe}$ & $2.04568 E+04$ \\
\hline $58 \mathrm{Fe}$ & $2.78986 \mathrm{E}+03$ & ${ }^{60} \mathrm{Fe}$ & $0.00000 \mathrm{E}+\infty 0$ & $57 \mathrm{Co}$ & $0.00000 \mathrm{E}+00$ \\
\hline${ }^{59} \mathrm{Co}$ & $2.17600 \mathrm{E}+03$ & $56 \mathrm{Ni}$ & $0.00000 \mathrm{E}+00$ & $58 \mathrm{Ni}$ & $3.46269 E+04$ \\
\hline${ }^{59} \mathrm{Ni}$ & $0.00000 E+00$ & $60 \mathrm{Ni}$ & $1.33646 \mathrm{E}+0.4$ & $61 \mathrm{Ni}$ & $6.11900 \mathrm{E}+02$ \\
\hline $62 \mathrm{Ni}$ & $-1.88669 E+03$ & $64 \mathrm{Ni}$ & $5.09917 E \div 02$ & & \\
\hline
\end{tabular}



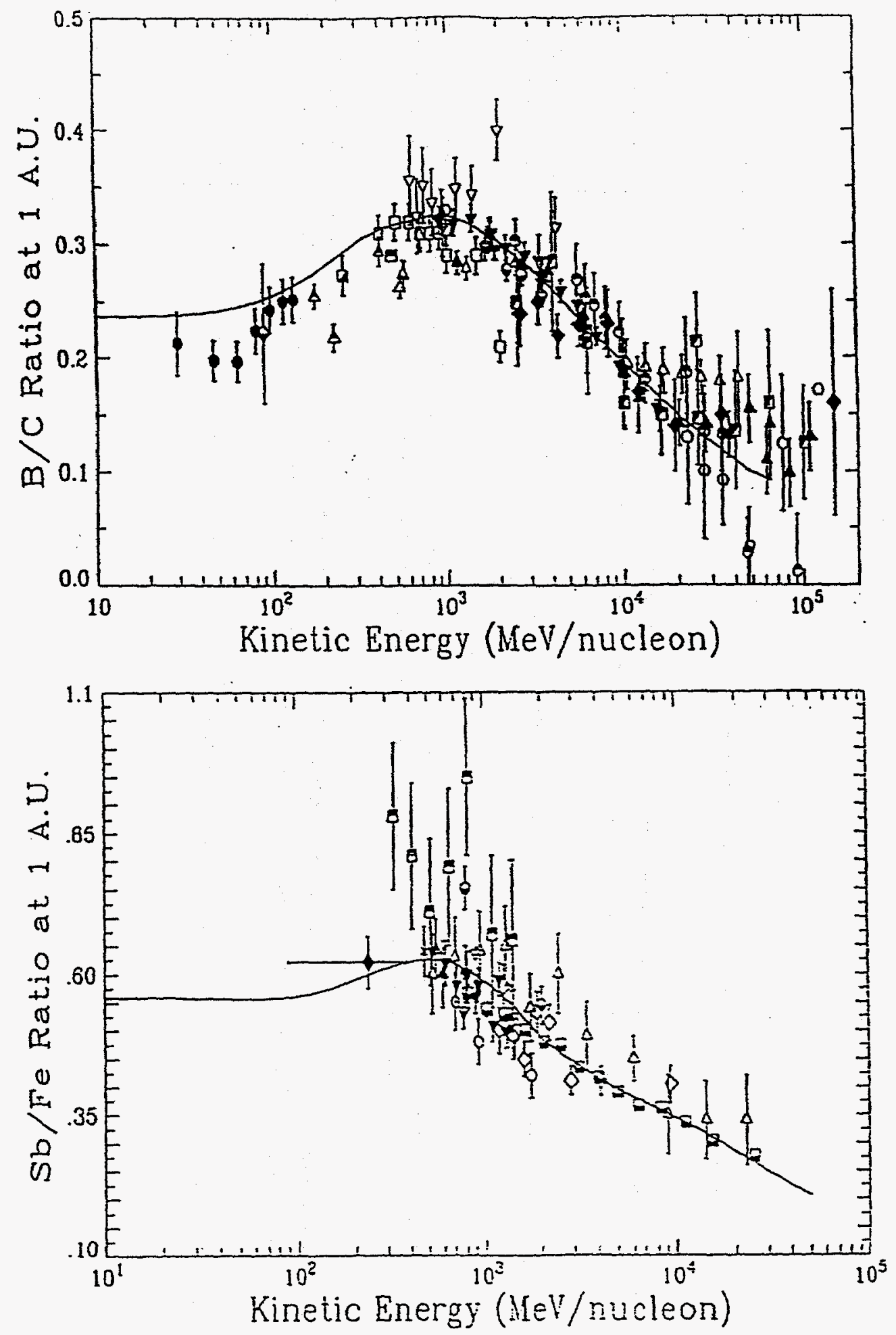

Figure 12: Measurements of the secondary to primary ratios $B / C$ (top) and $(\mathrm{Sc}+\mathrm{Ti}+\mathrm{V}+\mathrm{Cr}+\mathrm{Mn}) / \mathrm{Fe}$ (bottom) compared with the results of interstellar transport and solar modulation calculations. 
Since the Iron group species have a larger total nuclear interaction cross section, and hence a shorter mean interaction length, than the Carbon group nuclei, the iron secondaries will be more sensitive to the short pathlength characteristics of the PLD. Correspondingly, the $B / C$ will be sensitive to the longer pathlengths.

The resulting PLD is an exponential distribution of pathlengths with a characteristic $X_{0}$, shown as the solid curve of Figure 13, along with requiring that all cosmic rays pass through at least $X_{c}$ (dashed curve) amount of material. This PLD provides a reasonably good fit to the $B / C$ and Sub-Fe/Fe measurements as shown by the solid curves in Figure 12. Such a PLD may be consistent with propagation models which involve a "galactic wind" that flows outward from the galactic plane in a fashion similar to the solar wind which flows outward from the Sun (Garcia-Munoz et al., 1987).

The LIS spectra resulting from these propagation calculations are shown as the short dashed curves in Figure 14 for each element from $\mathrm{H}$ to Ni. These spectra need to be calculated only once since the variations in the GCR radiation environment are expected to be caused by "local", solar modulation effects. For comparison measurements from the GCR Database are shown in the Figure along with the energy spectra near the orbit of Earth (1 A.U.) for solar minimum (solid curve) and solar maximum (dashed curve) conditions. The GCR Database was initially developed in the early 1980's and a majority of the measurements available then were made during the extended solar minimum period of 19721978. Since then relatively few, new missions have been flown and the statistics collected during the current solar cycle have been hampered by the two relatively deep solar maxima bracketing a rather narrow solar minimum period (c.f. Figure 3). Thus, we would expect that the solar minimum curve would fit these data and this is indeed the case. The exceptions are elements for which only the Engelmann et al. (1990) measurements are available. These data were taken during 1979-1980 when solar modulation was increasing (c.f. Figure 3) and, consequently, a good fit would require a modulation level between minimum and maximum.

The difference between the solar minimum and solar maximum energy spectra below $1 \mathrm{GeV} /$ nucleon is quite large and for some elements this difference exceeds an order of magnitude at $10 \mathrm{MeV} /$ nucleon. Carefully accounting for the solar modulation effect is therefore important in accurately determining the interplanetary radiation environment. The CHIME model includes a series of spectra over a grid of solar modulation levels. The appropriate spectra are chosen based upon a "calibration" of the modulation level versus time. 


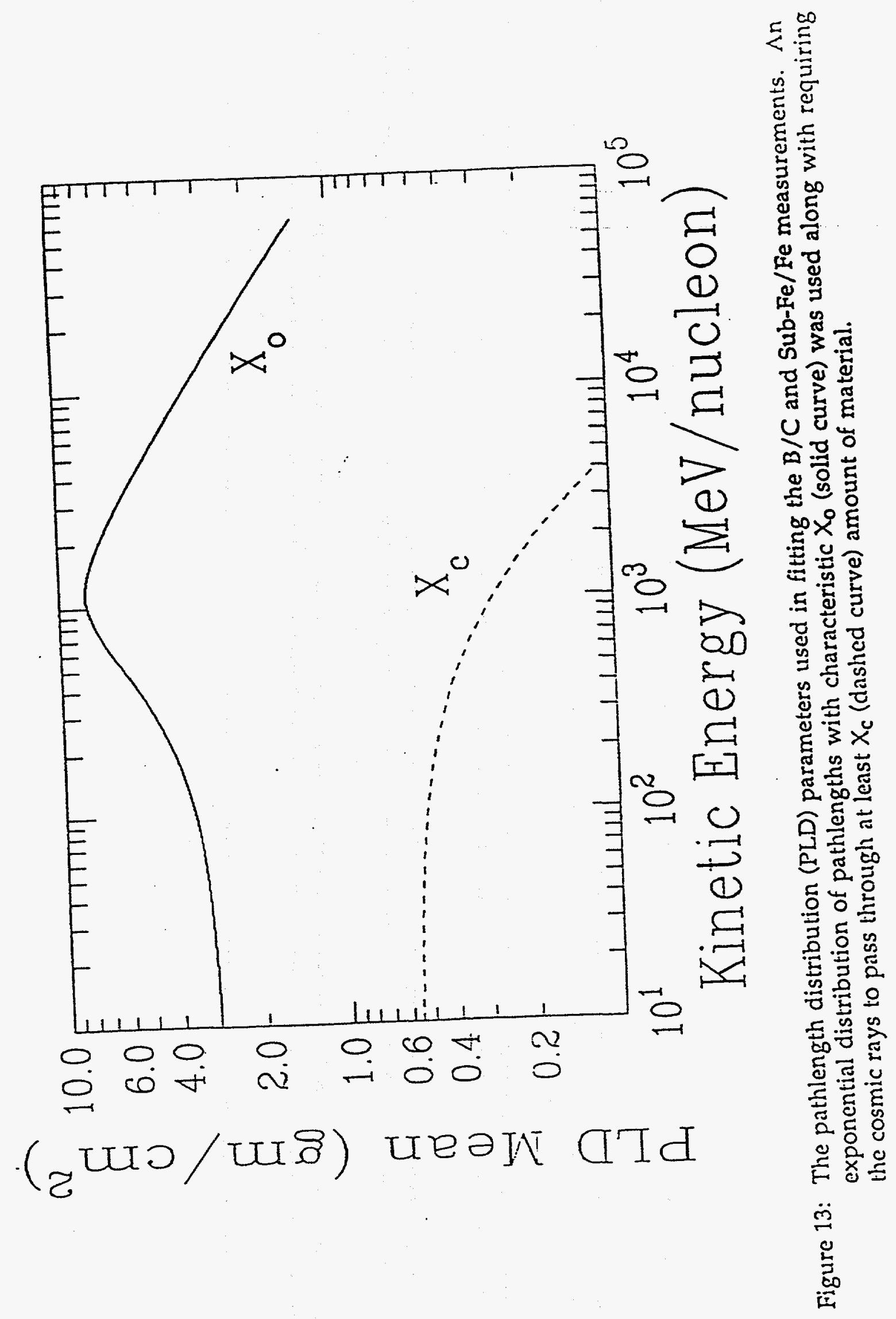




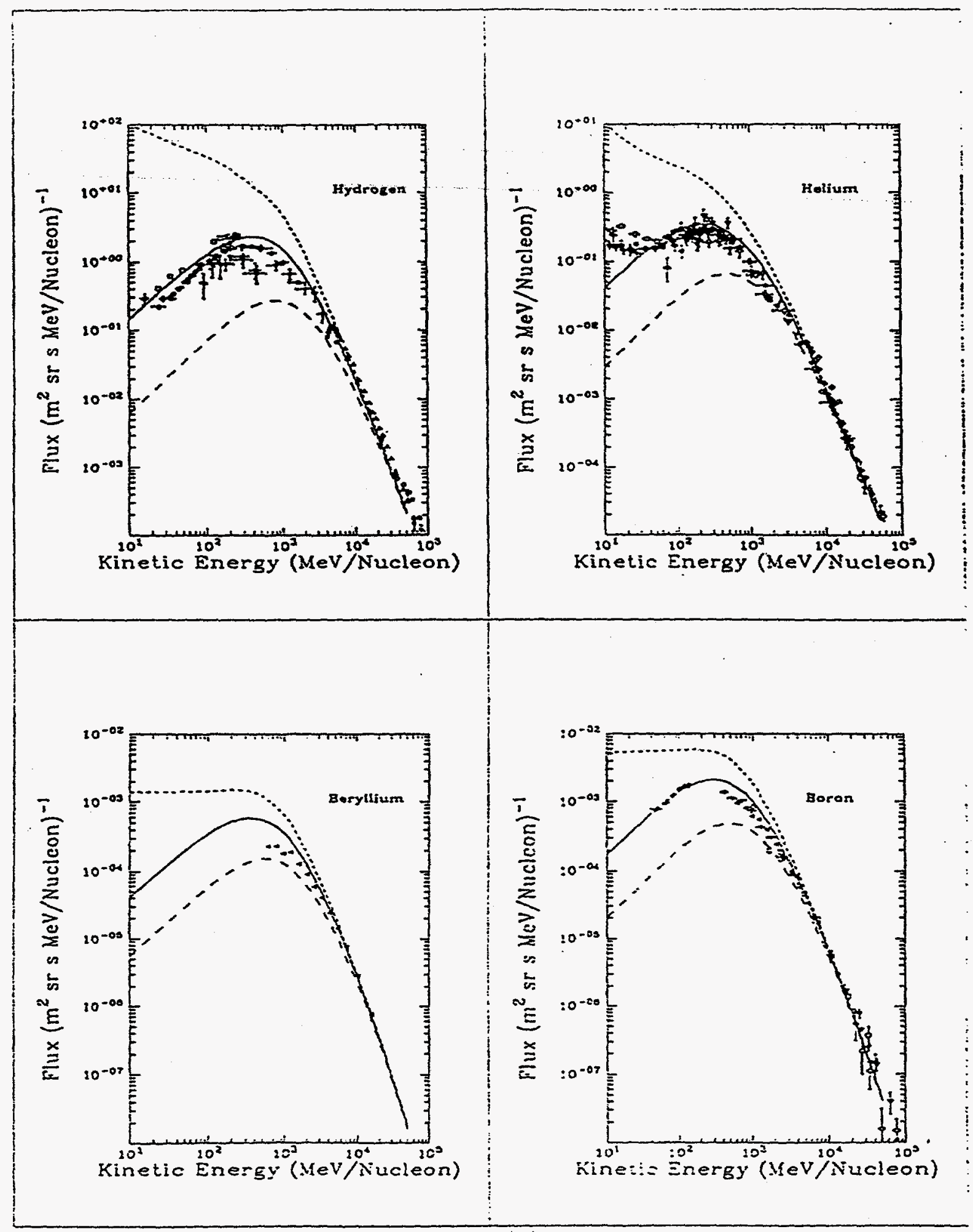

Figure 14: Differential energy spectra for the elements $\mathrm{H}, \mathrm{He}, \mathrm{Be}$ and $\mathrm{B}$ (see text for details). 


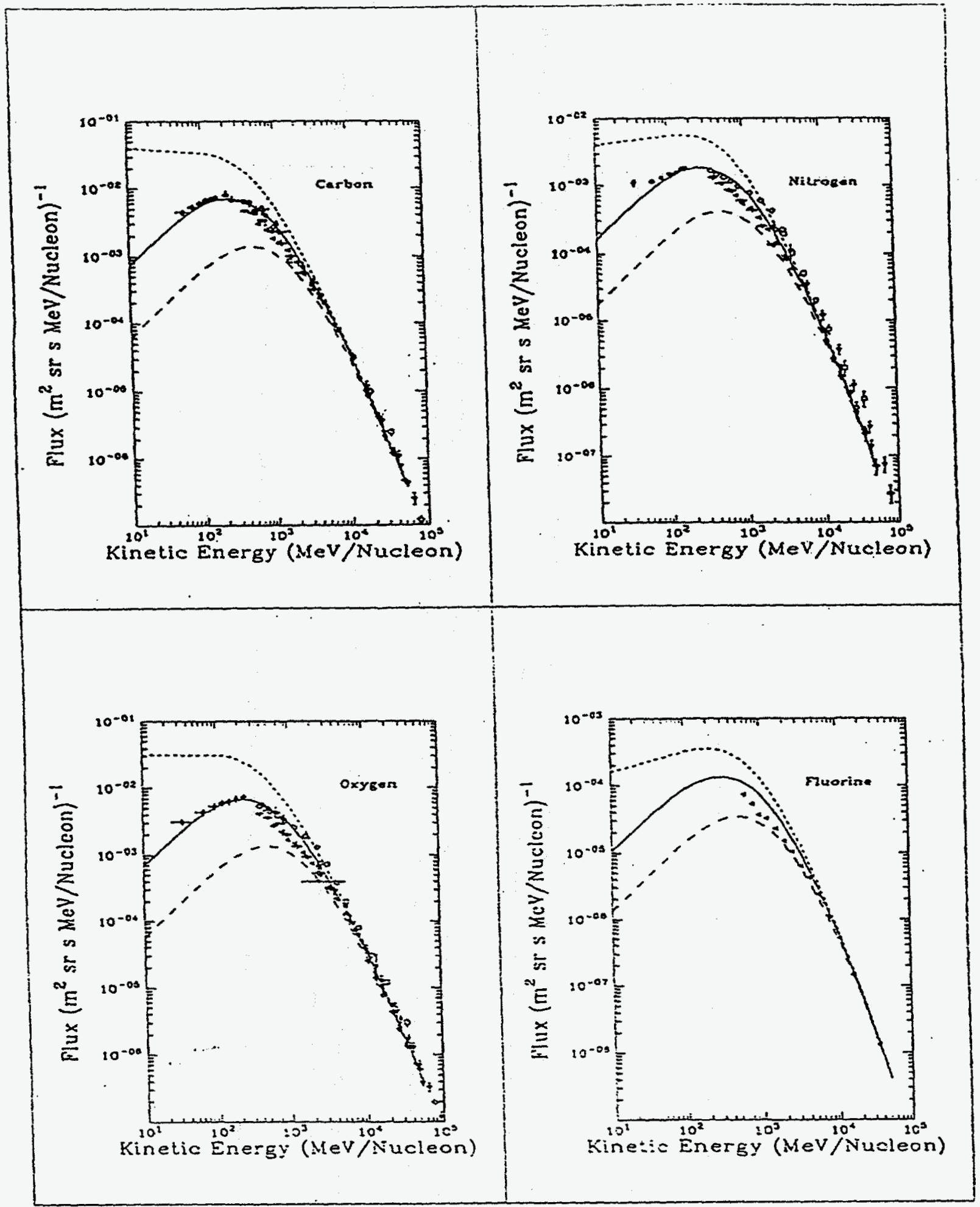

Figure 14 (cont): Differential energy spectra for the elements $C, N, O$ and $F$ (see text for details). 


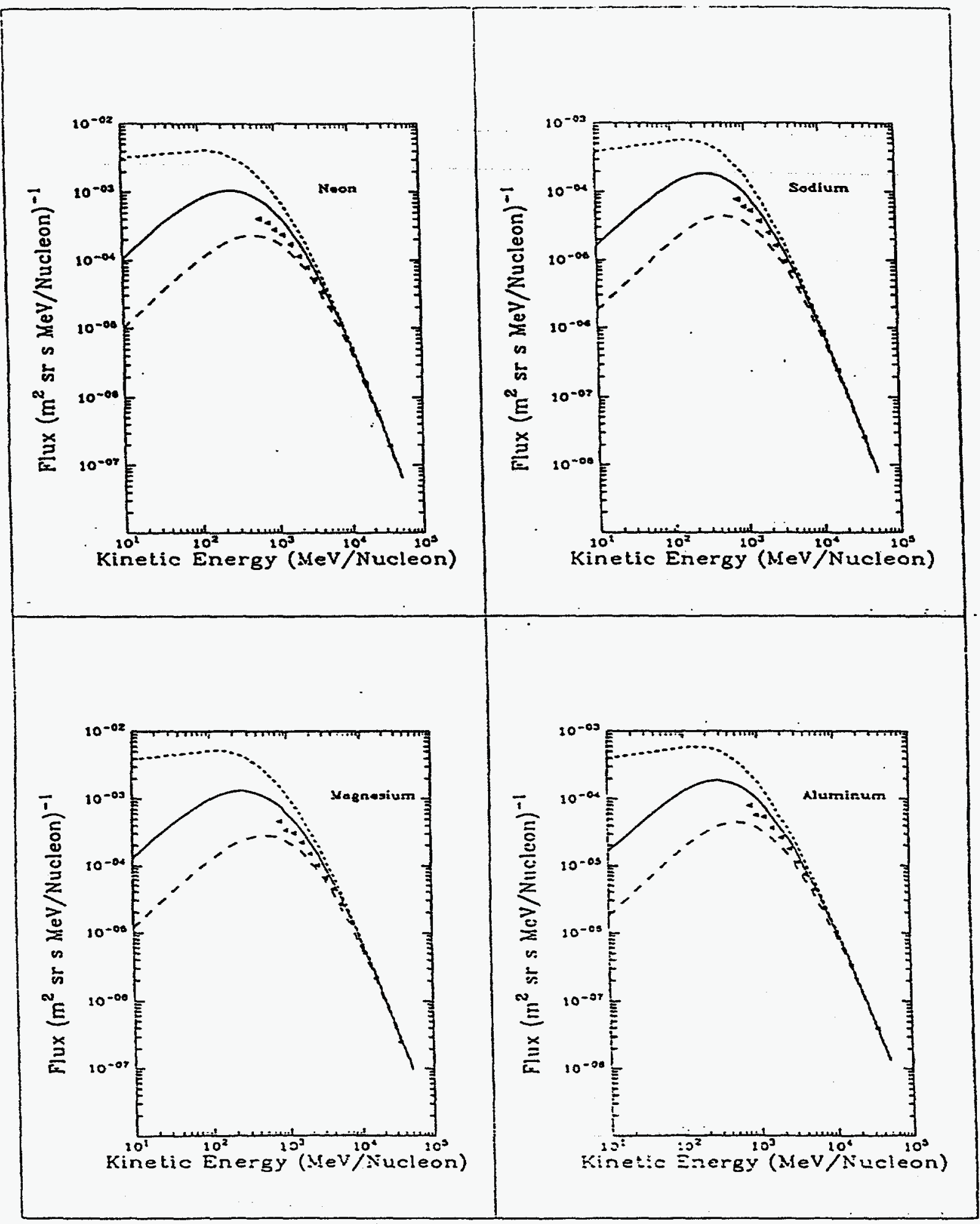

Figure 14 (cont): Differential energy spectra for the elements $\mathrm{Ne}, \mathrm{Na}, \mathrm{Mg}$ and $\mathrm{Al}$ (see text for details). 


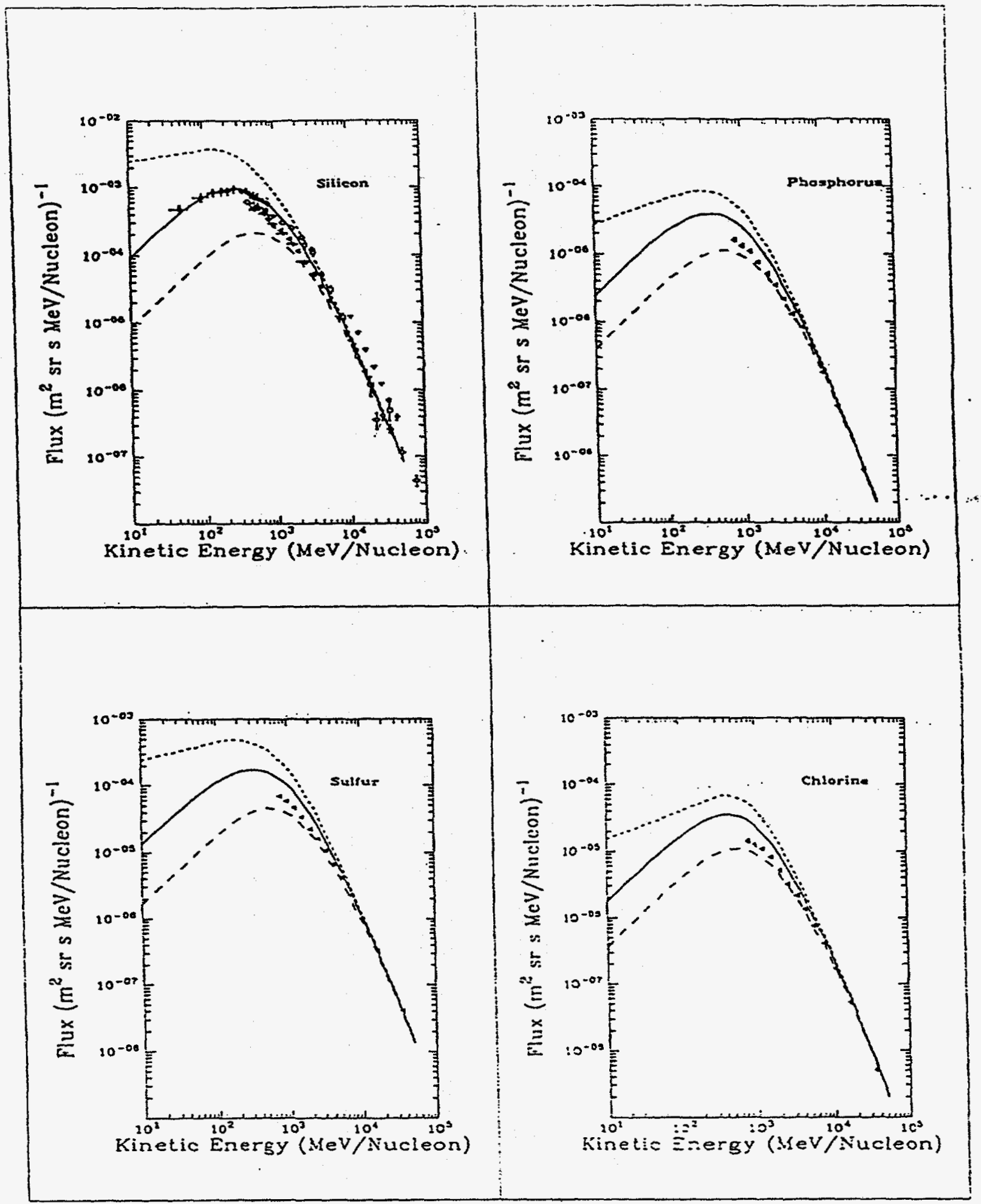

Figure 14 (cont): Differential energy spectra for the eiements $\mathrm{Si}, \mathrm{P}, \mathrm{S}$ and $\mathrm{Cl}$ (see text for details). 


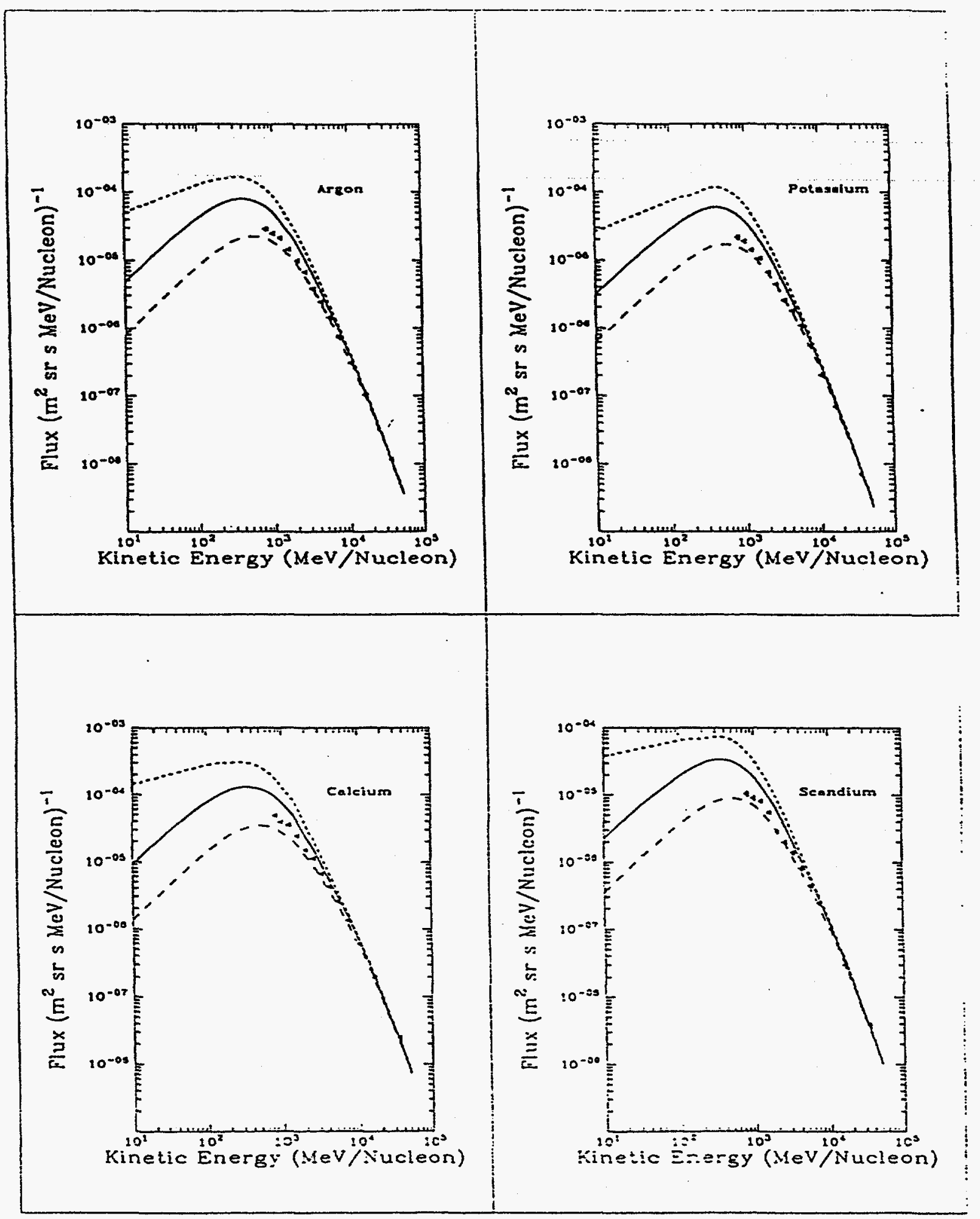

Figure 14 (cont): Differential energy spectra for the elements Ar, P, Ca and Sc (see text for details). 


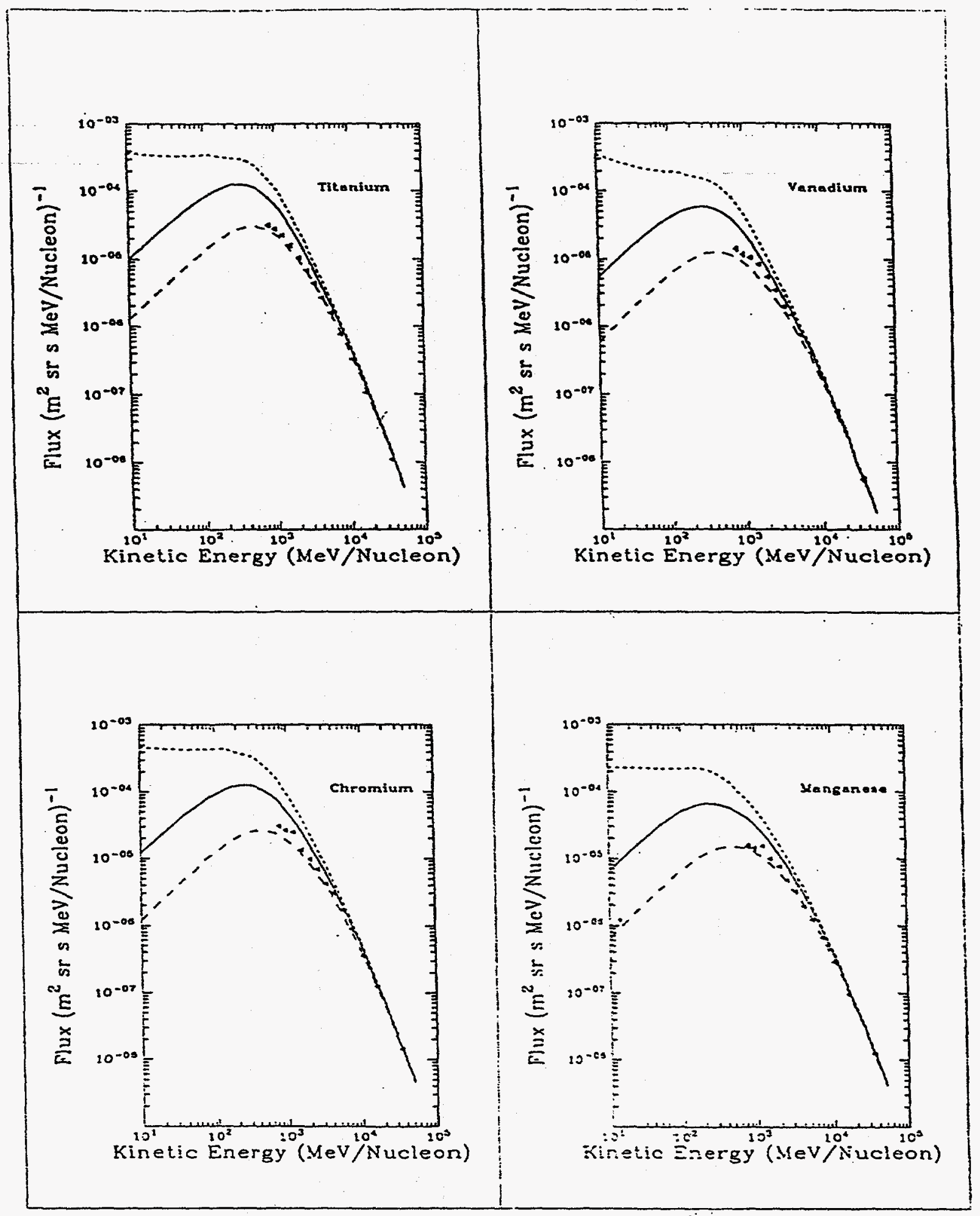

Figure 14 (cont): Differential energy spectra for the elements $\mathrm{Ti}, \mathrm{V}, \mathrm{Cr}$ and $\mathrm{Mn}$ (see text for details). 


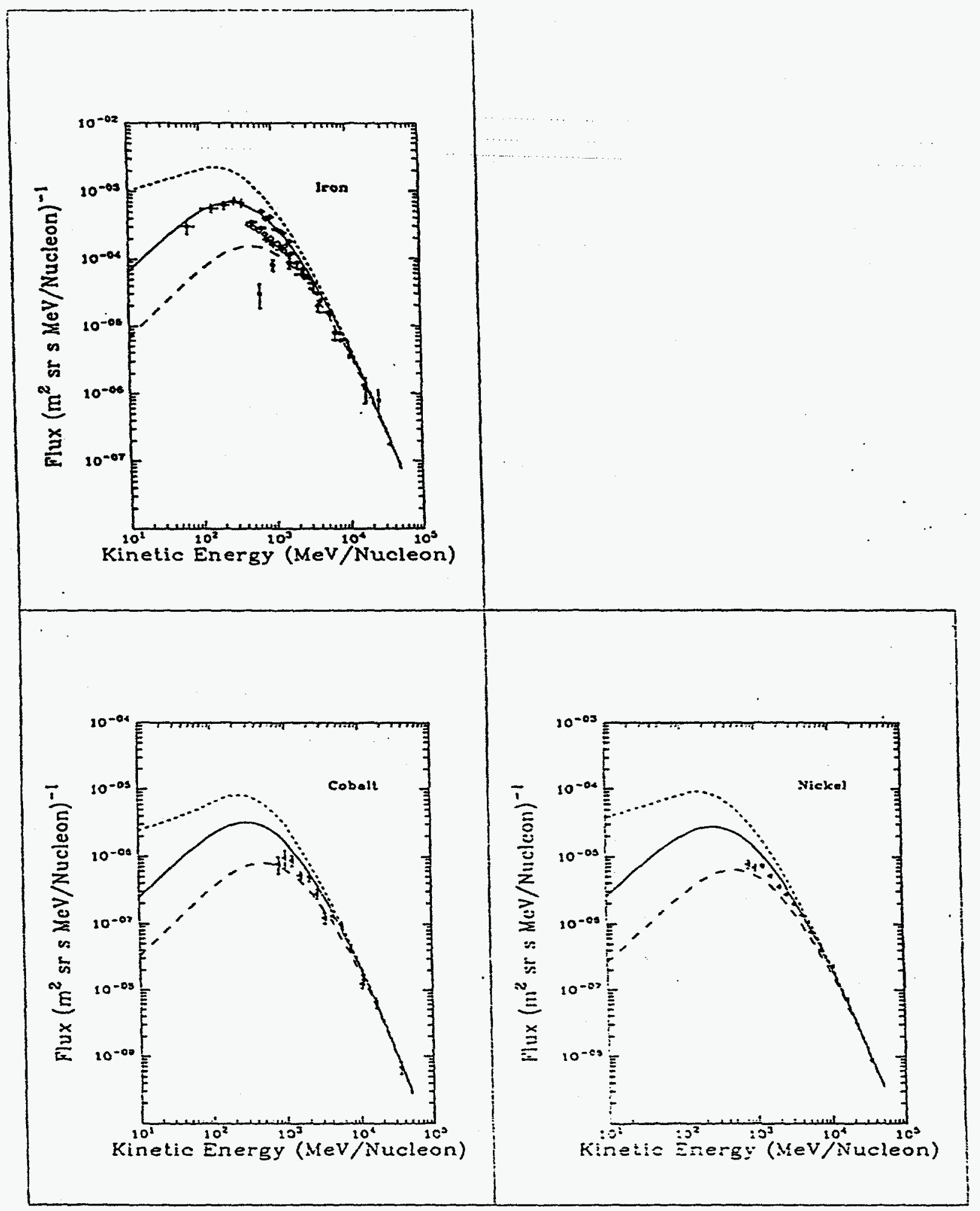

Figure 14 (cont): Differential energy spectra for the elements $\mathrm{Fe}, \mathrm{Co}$ and $\mathrm{Ni}$ (see text for details). 
For our present work, it was decided to develop AC local interstellar spectra only for helium, nitrogen, oxygen, and neon. The observed $A C$ fluxes for carbon and especially argon are quite low, and the inclusion of anomalous components of these species would not be significant. The AC contribution is more significant for the remaining four elements, and in particular, it is important to include the AC helium in the analysis of the solar modulation level as a function of time, as described in the next section. For each of the four elements included, the model AC spectrum contains only the primary isotope (i.e., ${ }^{4} \mathrm{He},{ }^{14} \mathrm{~N},{ }^{16} \mathrm{O}$, and ${ }^{20 \mathrm{Ne}}$ ). Contributions from other isotopes would be very small compared to the primary isotope, and considering that the AC flux itself is comparatively small except at the lowest energies, the added refinement of attempting to model the isotopic composition is not warranted at this stage.

The helium and oxygen local interstellar spectra were derived at the University of Chicago, by demodulation of measured data (Garcia-Munoz, private communication) and have been adopted for this analysis. The LIS for nitrogen and neon were obtained by renormalizing the oxygen spectrum, with the normalization factor chosen to yield the best fit obtainable to a variety of measured $\mathrm{AC}$ datasets corresponding to different levels of modulation and different locations in the heliosphere. It was possible to obtain reasonable fits to the datasets for these three elements at energies above about $10 \mathrm{MeV} /$ nucleon using just a single spectral shape, that of the demodulated oxygen spectrum. Measurements from IMP-8 at 1 AU, the Voyager spacecraft at 2 and at $21 \mathrm{AU}$, and Pioneer 10 at $16 \mathrm{AU}$ were used in fitting the $\mathrm{AC}$ spectra.

Figure 15 shows the Local Interstellar Spectra (LIS) that have been adopted for the four anomalous elements that have been studied. The LIS are cut-off at 5 $\mathrm{MeV} /$ nucleon, but the $\mathrm{N}, \mathrm{O}$ and $\mathrm{Ne}$ would be expected to turn-over, like the $\mathrm{He}$, at lower energies. Modeling this turnover has not been possible within the available data. Figures 16 and 17 show the results of our predictions, using the spectra of Figure 15 as input. The LIS were modulated to the time interval and the location in the heliosphere of the spacecraft from which the data were reported. The agreement between the predictions (solid lines) and the data points is reasonably good except for Voyager II results from 1985-86 at 21 AU for $\mathrm{N}$ (Figure $17 \mathrm{~B}$ ) and $\mathrm{O}$ (Figure $16 \mathrm{D}$ ) below $10 \mathrm{MeV} /$ nucleon. The equivalent data for anomalous $\mathrm{Ne}$, however, is in good agreement with the predictions. Whether this indicates a problem with the $N$ and $O$ data during 1.985-86 or with the predictions is unknown. The agreement for $\mathrm{E}>10 \mathrm{MeV} /$ nucleon is, however, good enough for the purposes of the present study.

Note that the $\mathrm{AC}$ is singly charged and has, therefore, a higher rigidity than a Galactic Cosmic Ray (GCR) ion of the same energy. In order to combine the $A C$ spectra with the GCR spectra, a routine was written to generate a binary file that mimics the output of the GCR propagation calculation. The solar 


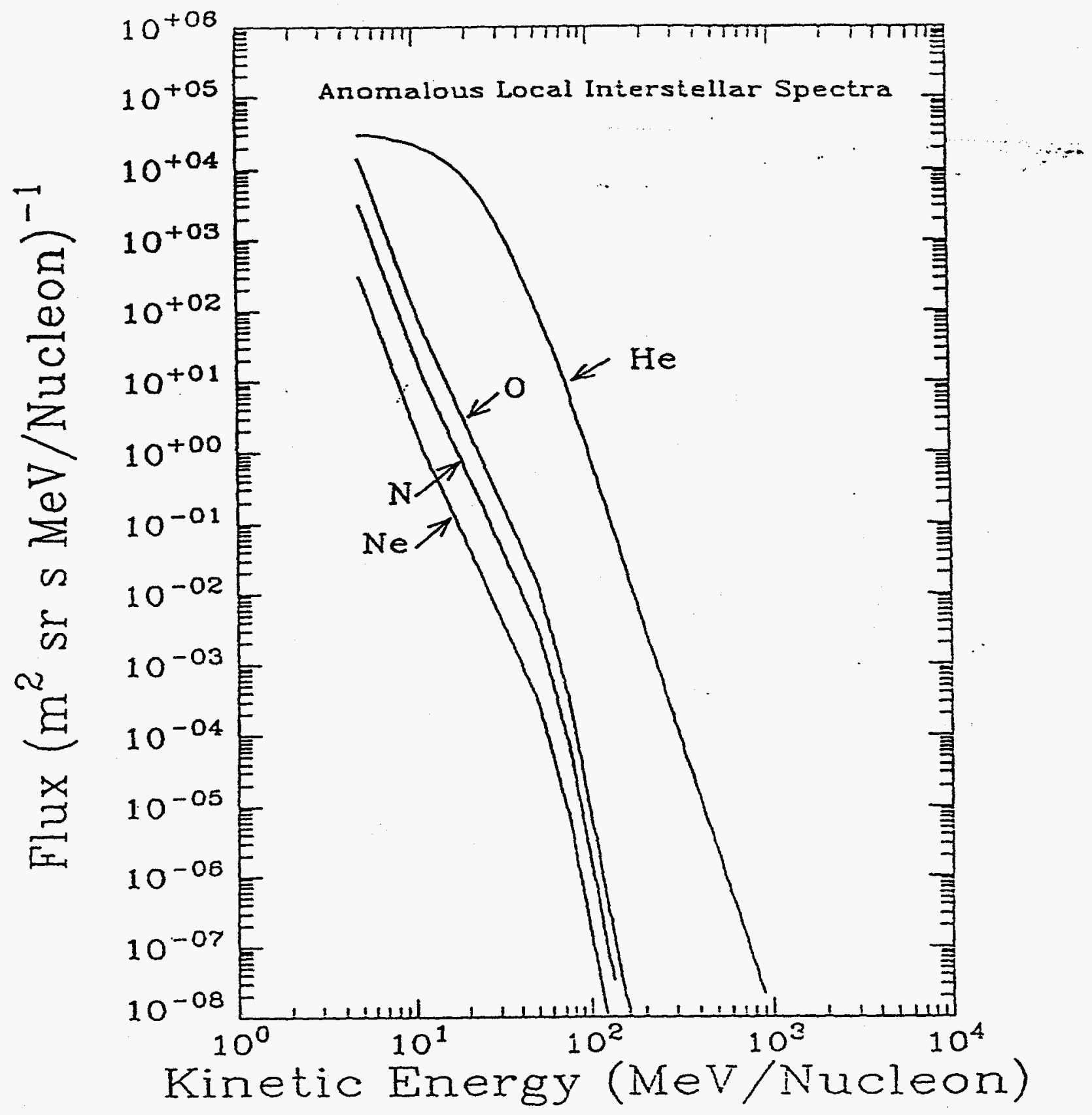

Figure 15: The Anomalous Component local interstellar spectra for $\mathrm{He}, \mathrm{N}, \mathrm{O}$ and $\mathrm{Ne}$ used in the Model. The Helium anc Oxygen spectra were both derived by demodulating anomalous component measurements, while the LIS for $\mathrm{N}$ and $\mathrm{Ne}$ were obtained by scaling the Oxygen spectra. 


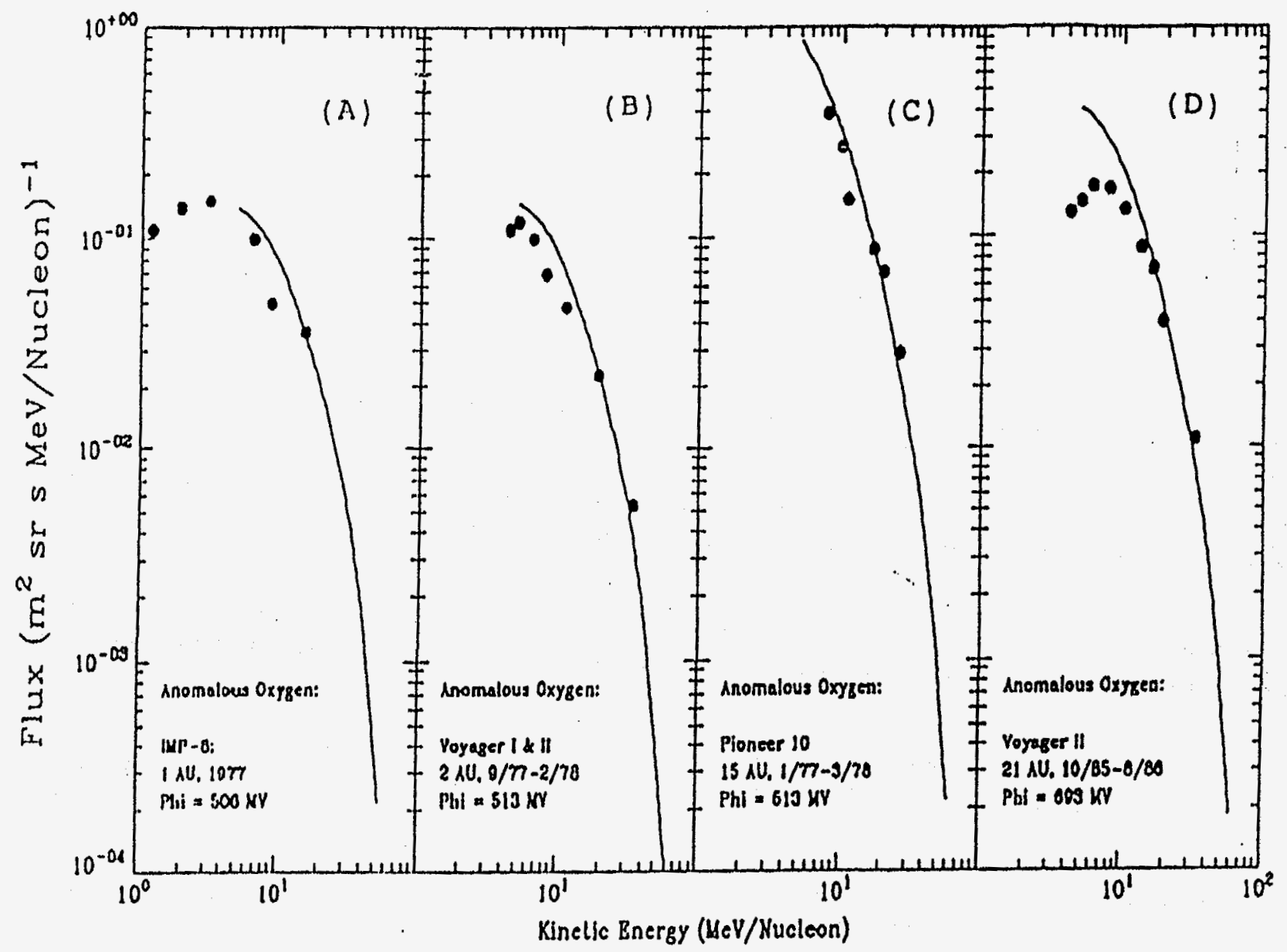

Figure 16: Anomalous Oxygen measurements (filled circles) made at different times and at different locations in the heliosphere compared to the GCR Model calculations (solid lines). From left to right the measurements were made by IMP-8 at 1 A.U. during 1977, by Voyager I \& II at 2 A.U. during $9 / 1977-2 / 1978$, by Pioneer 10 at 15 A.U. during $1 / 1977-3 / 1978$, and by Voyager II at 21 A.U. during 10/1985 - 8/1986. Each calculated spectrum used the same LIS, but with heliospheric radii and solar modulation level $(\phi)$ appropriate to the given data. 


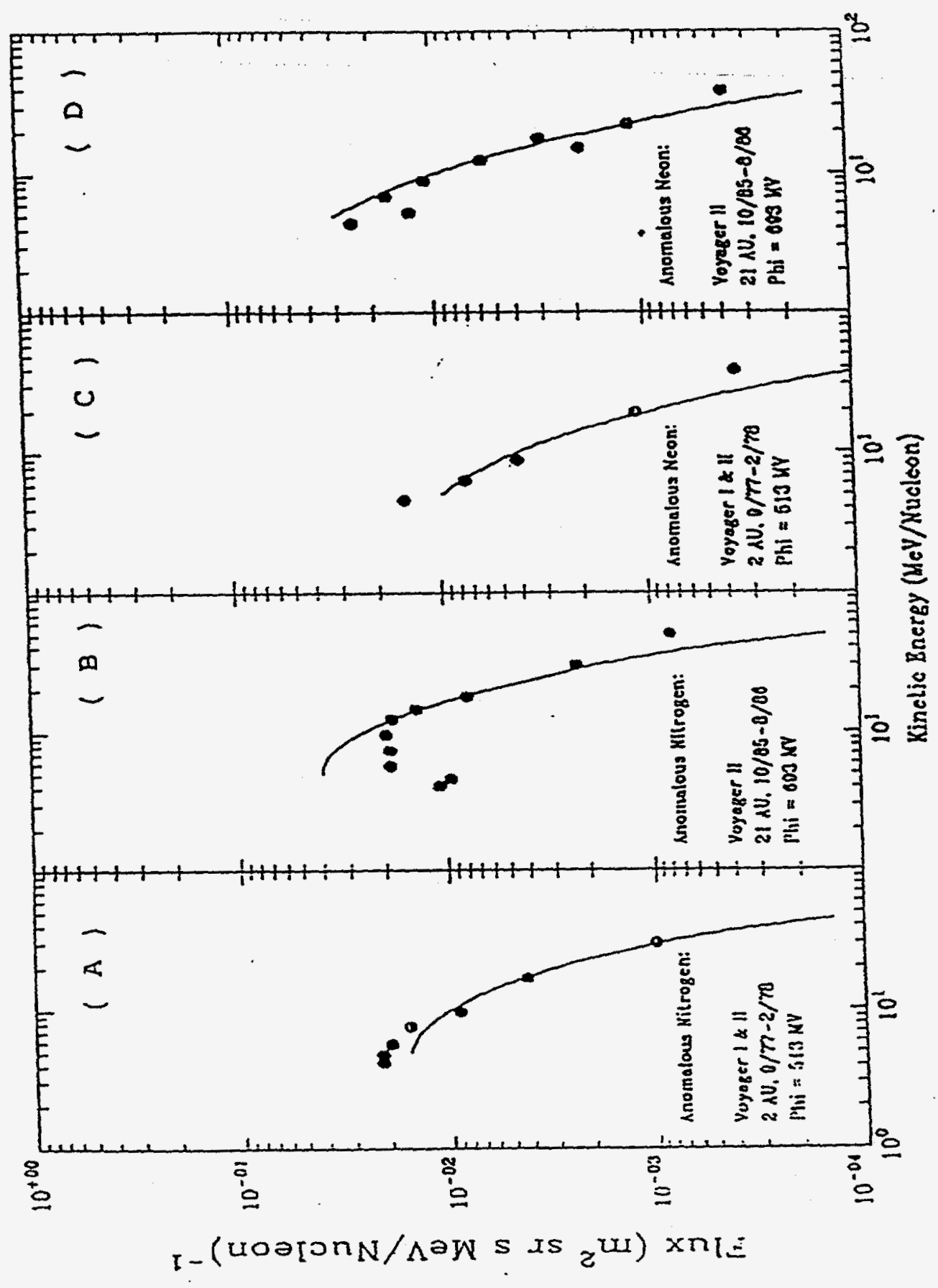

혹

อิ

을

을

空要

8

ن

灵

放

$\ddot{\Xi}$

范 
modulation program accepts this file, modulates the elements with a charge of +1 , then outputs the spectra on the same energy grid as the GCR spectra. The modulated AC spectra can then be added point-by-point to the GCR spectra, if desired. It is important to note that the AC must be modulated separately from the GCR component, because of its singly-charged ionization state.

\section{Solar Modulation}

It is well known (see reviews by Jokipii 1971, Fisk 1979, McKibben, 1986) that at energies below a few $\mathrm{GeV}$ per nucleon the intensity and the spectral shape of the cosmic rays arriving at the orbit of Earth are significantly modified by solar modulation. The cosmic rays diffuse into the heliosphere against the outward-flowing solar wind, carrying frozen-in interplanetary magnetic field, which convects the cosmic rays out of the heliosphere. In this process, the cosmic rays lose energy to the expanding field (adiabatic deceleration), and their energy spectrum is modified.:

A spherically symmetric model of solar modulation has been developed (Parker 1965; Jokipii 1971; Urch and Gleeson 1972; Fisk 1979) which explains most of the gross features of the modulation process. This model includes the effects of diffusion, convection, and adiabatic deceleration (but not drifts due to the gradient and curvature of the magnetic field) and assumes that these three physical processes are in equilibrium in the heliosphere. Quantitatively these effects are represented by a Fokker-Planck equation in which the parameters are the solar wind velocity, the diffusion coefficient and the radius of the heliosphere, with the cosmic-ray differential energy spectrum in local interstellar space as a boundary condition.

Evenson et al. (1983) (see also, Garcia-Munoz et al., 1986), solving this equation numerically, have analyzed the simultaneous modulation of electrons, protons, and helium nuclei over the 1965-1979 period involving more than one solar cycle. They find that in general the model fits the data quite well. In this model, the degree of modulation at a heliospheric radius $r$ is given by the modulation parameter

$$
\phi(r)=\frac{1}{3} \int_{r}^{R} \frac{V\left(r^{\prime}\right)}{K\left(r^{\prime}\right)} d r^{\prime}
$$

where $V\left(r^{\prime}\right)$ is the solar wind velocity, $K\left(r^{\prime}\right)$ is the radial part of the diffusion coefficient, and $R$ is the radius of the heliosphere. An insight into the physical meaning of $\phi$ is obtained from the "force-field" approximation (Gleeson and Axford 1968) in which $\Phi$ corresponds to a "potential energy," that in the particular case in which the diffusion coefficient is proportional to particle rigidity takes the simple form 


$$
\Phi=|Z e| \phi(r),
$$

where $\mathrm{Ze}$ is the particle charge. This potential energy has been identified as the mean energy loss that the particles experience in penetrating the heliosphere to a radius $r$. The parameter may be expressed in rigidity or energy units, connected through,

$$
\phi(\mathrm{MV})=(\mathrm{A} / \mathrm{Z}) \Phi(\mathrm{MeV} / \text { nucleon })
$$

The modulated nucleonic differential energy spectra obtained by the numerical solution of the Fokker-Planck equation are almost completely determined by the value of the modulation parameter $\phi$. Combinations of the parameters $R, r, V(r)$, and $K(r)$ giving the same value of $\phi$ will lead to modulated nucleonic spectra which are very nearly equal to each other (Urch and Gleeson 1972). Therefore, values of the'modulation parameter $\phi$ can be used to specify different levels of modulation.

Our approach to determine $\phi$ as a function of time is to use the $70-95$ $\mathrm{MeV} /$ nucleon Helium flux measured by the University of Chicago instrument on-board the IMP-8 spacecraft over the time period 1974 to 1993 as a "standard litmus" for the modulation level. This low energy Helium is very sensitive to the exact level of solar modulation, and the raw data, including SEP event rate spikes, is shown in Figure 18. The underlying solar cycle variation is evident. However, to obtain an unbiased $\phi$ estimate, the flare spikes were removed and the data was re-binned into monthly averages. This "quiet-time" 70-95 $\mathrm{MeV} /$ nucleon Helium data is shown in the middle panel of Figure 19. The top panel of this figure shows the Climax neutron monitor data for the same time period and these two independent measures of solar modulation track each other quite well.

Starting with our Local Interstellar Space (LIS) helium spectrum, modulation calculations were performed as a function of the modulation parameter. As it is possible that the He measurements might contain "contamination" from Anomalous Component $\mathrm{He}$, both the GCR and AC He LIS were modulated using the appropriate charge state and then combined. These spectra were then integrated between 70 and $95 \mathrm{MeV} /$ nucleon to produce a flux versus $\phi$ calibration curve. This is then applied to the measured "quiettime" He to derive the $\phi$ vs time plot shown at the bottom of Figure 19. In this fashion we can determine $\phi$ for every month from Novemiber 1973 to May 1993. The uncertainties reflect the errors in the measurement of the $70-95$ $\mathrm{MeV} /$ nucleon Helium and are representative of the level of uncertainty that exists for a monthly average. 


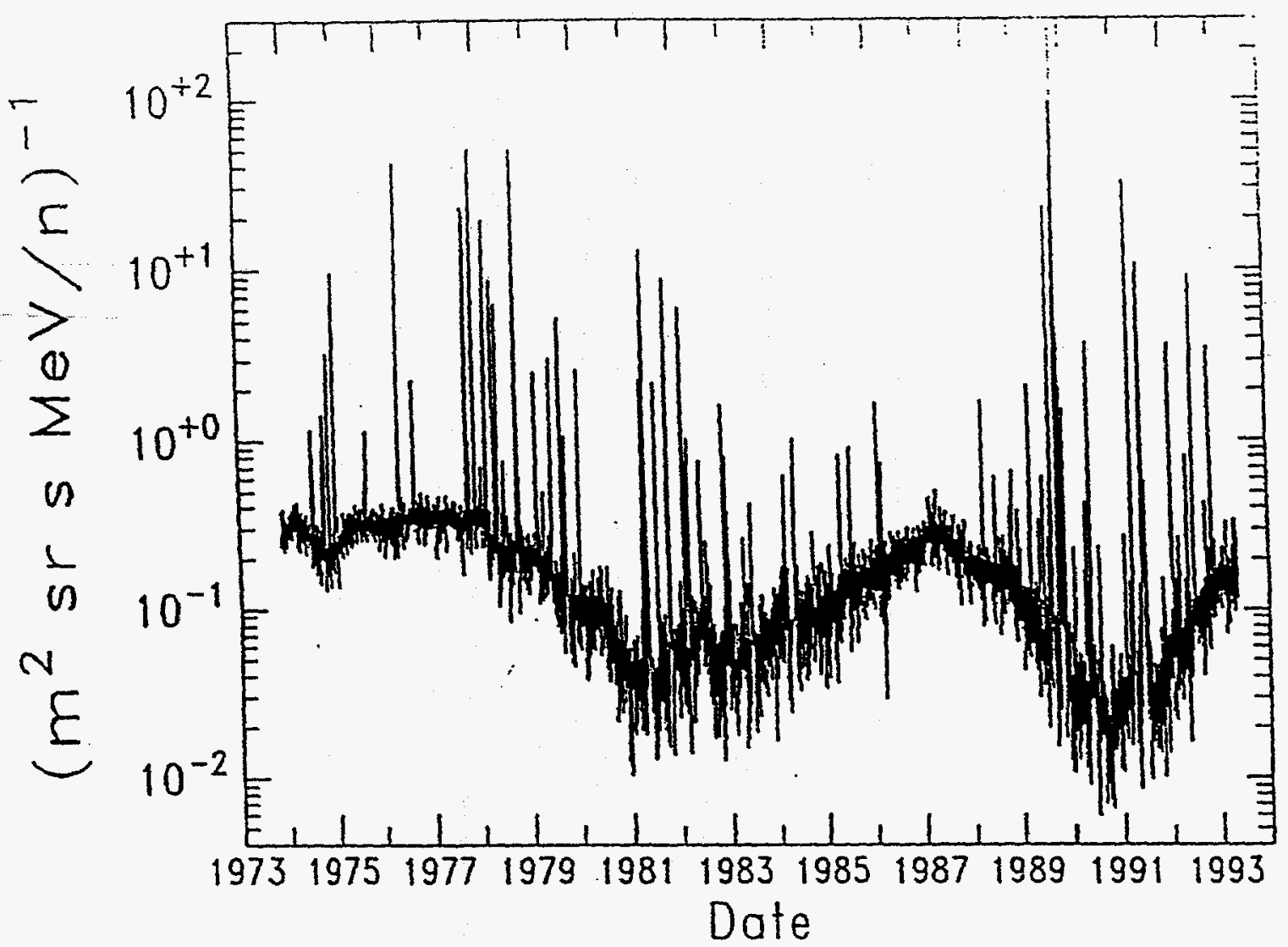

Figure 18: Daily average flux of 70-95 MeV/nucleon Helium as measured by the IMP-8 satellite from 1973 to 1993 . The solar cycle variation is clearly seen as the broad, dark band with solar energetic particle (SEP) active times (sharp spikes) superimposed. Very few SEP events occur during solar minimum periods, but can occur for several years after solar maximum. Note that during 1992 and 1993 the quiet time Helium flux is retuming to a solar minimum level. This trend is expected to continue during 1994.

In order to predict future levels of modulation, the assumption was made that the modulation level will be periodic, following the 22-year solar cycle. To avoid predicting exactly the same transient features seen in the $\phi$-versus-time plot of Figure 19, the set of monthly average o values from 1973-93 was smoothed using a Fast Fourier Transform method, and the smoothed set of values was then translated 22 years into the past and 22 years in to the future to provide estimated monthly average values. The values of 0 for the interval January 1970 through May 1971 are, therefore, assumed to reflect the values from January 1992 through May 1993. Likewise, $\phi$ from November 1995 through December 2010 can be obtained from the corresponding values for November 1973 (when the IMP-8 helium data began) through December 1988. Most of the 


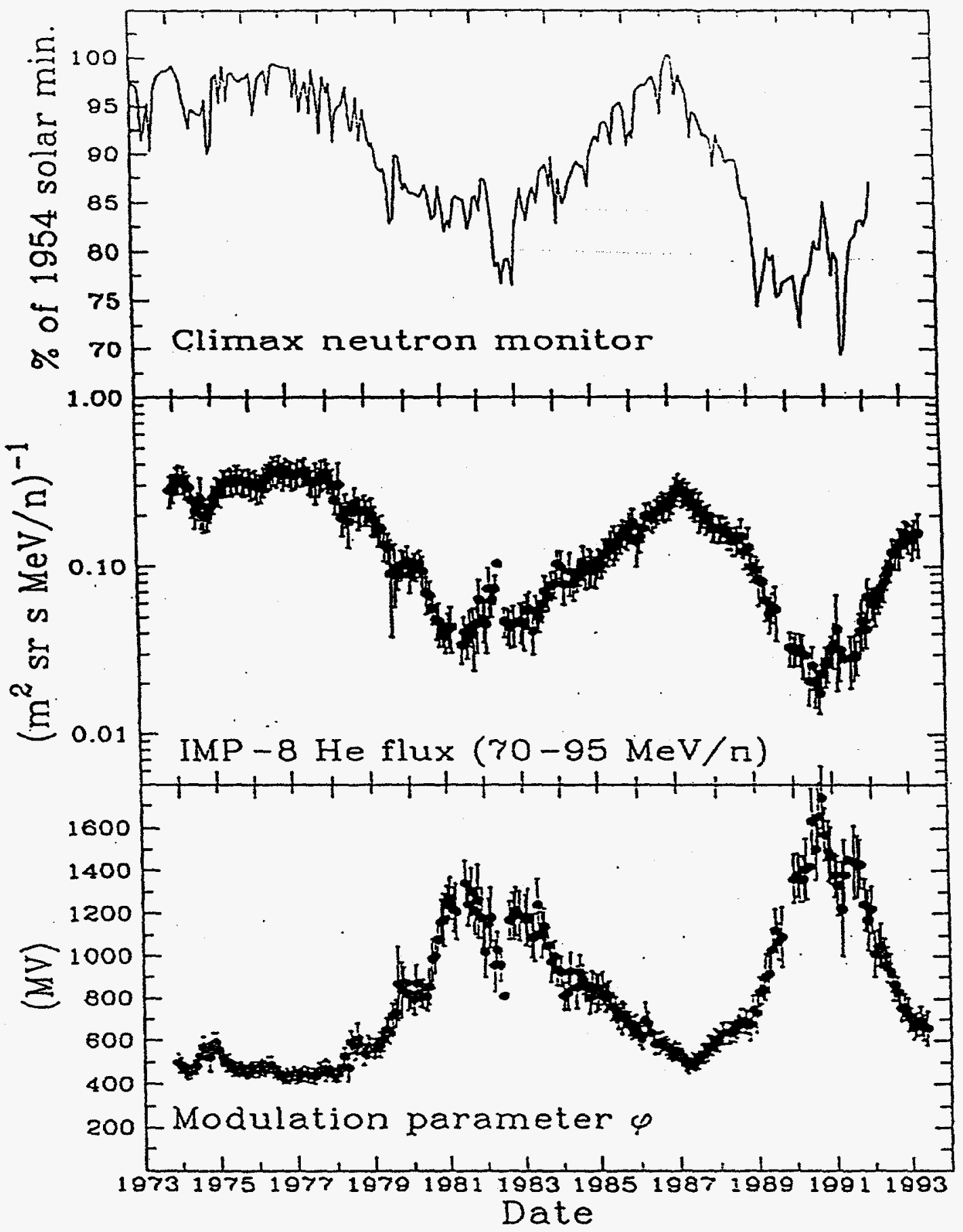

Figure 19: The time variation of solar modulation from 1973 to 1993. The top panel shows the Climax neutron monitor rate which tracks with the monthly averaged 70-95 MeV/nucleon IMP-S Helium, cleaned of SEP active times, (middle panel). The solar modulation parameter ( $\phi)$ derived from the Helium flux is shown in the bottom panel. For solar minimum $\phi$ is about $450 \mathrm{MV}$, while for solar maximum $\phi$ can be as large as 1500 to $1600 \mathrm{MV}$. 
40 year interval 1970-2010 can thus be filled by using the helium-based $\phi$-versustime profile in Figure 19. However, there remain two gaps of identical length, from June 1971 through October 1973 and from June 1993 through October 1995, reflecting the fact that the IMP-8 data available at present fall 29 months short of spanning a full 22-year solar cycle.

As we have shown previously, there is a good correlation between the modulation level and the Climax (or any other) neutron monitor counting rate, for which data exist back to the 1950's. Therefore, we used the neutron monitor for the interval 6/71-10/73 as a guide in filling the remaining gaps. We assume that the overall qualitative behavior of the neutron monitor and the modulation will be similar over any time interval not chosen too short. Even over spans of months, there are changes in neutron monitor rate that are not immediately mirrored in the helium fluxes, and vice versa. Therefore, the neutron monitor information was used only as a check to help ensure that the $\phi$ values estimated to fill the gaps do not fail to reflect any major trends. The neutron monitor data was smoothed using the same method mentioned previously, so that just the qualitative trend of the neutron count rate can be seen. Monthly values of the modulation level for $6 / 71-10 / 73$ and $6 / 93-10 / 95$ were then fitted into the gaps by hand, attempting more or less to reproduce the trend of the neutron monitor data and avoid any discontinuities in the modulation level at either end of the gaps.

The resulting total set of monthly average levels of modulation for the entire interval 1970-2010, with both remaining gaps filled, is shown in Figure 20. This extrapolation captures well the differences between even - and oddnumbered solar cycles which have been attributed to the effects of particle drifts due to the change in polarity of the solar and interplanetary magnetic field (Kota and Jokipii, 1983). It predicts, for example, a long period of solar minimum conditions, like that of $1973-1978$, and a relatively broader but less intense maximum in solar modulation, peaking in 2003.

Figure 20 is now the database used for calculating average values of the level of modulation over any specified time interval. A user of the CHIME model may select either a time period or a specific solar modulation level to specify the GCR and AC flux. If a time period is selected, the software computes the average of the flux over the specified time period, using the monthly values of the solar modulation parameter. 


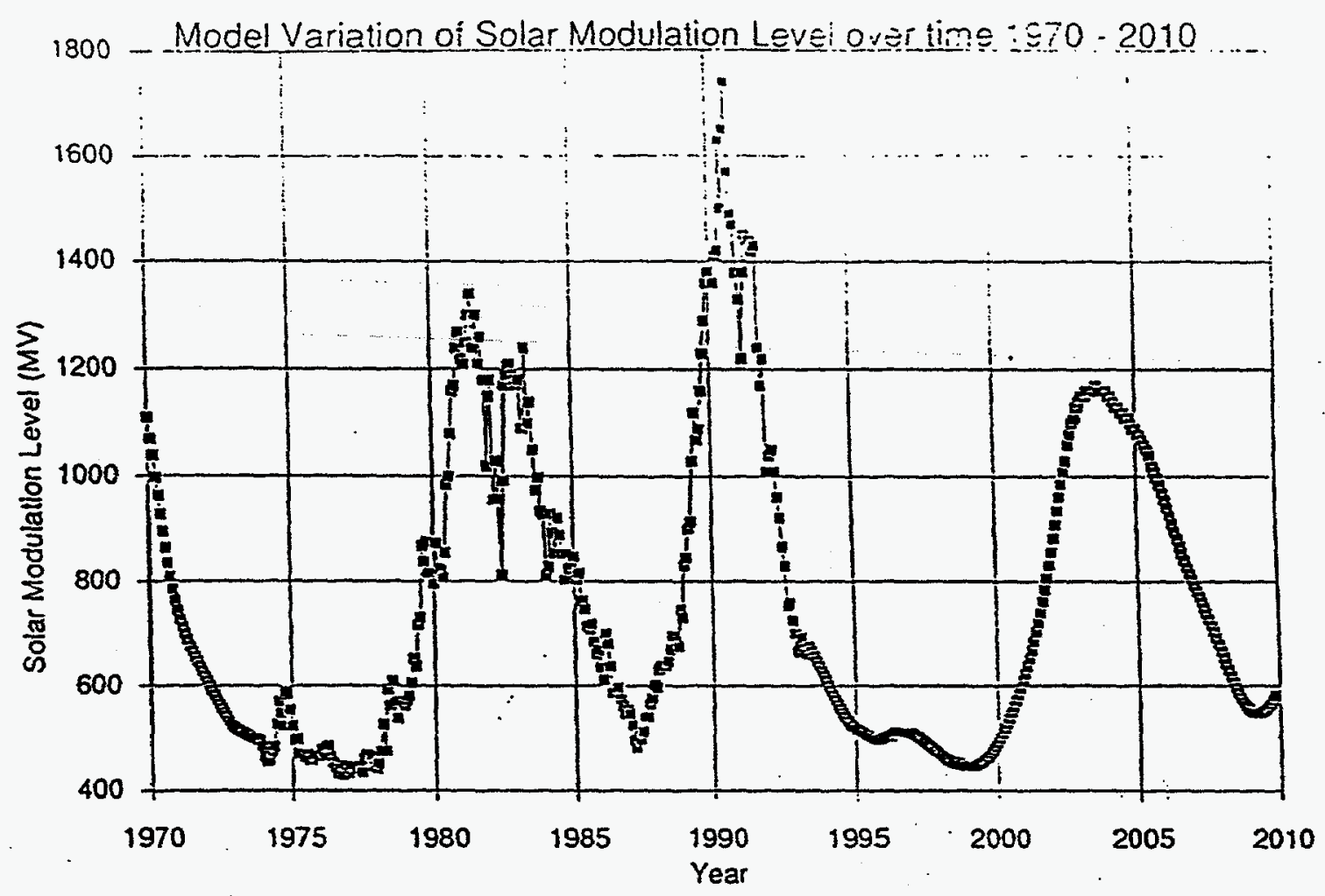

Figure 20: The solar modulation parameter, $\phi$, used in the GCR Model covering the period 1970 to 2010 . The $\phi$ derived from the IMP-8 Helium flux is used for the period 1973 to 1993 . Smoothing this data and translating it 22 years (one complete solar cycle) forward and backward in time provides a prediction for the extended period. Note that this method predicts a solar minimum period lasting from -1995 to 2000 .

\section{Solar Energetic Particle Events}

The CHIME model uses a set of SEP events observed during the CRRES mission as a sample of the SEP fluence. For a given time period, the total SEP fluence of all species can be calculated, and added to the GCR fluence, to look at effects that might be produced in a specific instrument or microelectronic device. From the modeling viewpoint, the important parameters of an event are the proton and helium energy spectra, the heavy ion enrichment, if any, and the total duration of the event. Average properties are determined by using all of the events that are SEP related. Afterwards; corrections are made for the Galactic Cosmic Ray (GCR) background within the flare period.

Table 2 summarizes the twenty-six events which occurred during the CRRES mission and which have been characterized. For some of the flares early in the mission, there were data coverage gaps that prevented determination of some of the identifying parameters. In particular, not all of these smaller SEP's can be correlated with known flares and may be due to non-flare Coronal Mass 
TABLE 2. SEP Events During the CRRES Mission

\begin{tabular}{|c|c|c|c|c|c|c|c|c|c|}
\hline $\begin{array}{l}\text { Event } \\
\text { Number }\end{array}$ & $\begin{array}{l}\text { Peak } \\
\text { Orbit }\end{array}$ & $\begin{array}{l}\text { Start } \\
\text { (Day) }\end{array}$ & $\begin{array}{l}\text { Peak } \\
\text { (Day) }\end{array}$ & $\begin{array}{l}\text { End } \\
\text { (Day) }\end{array}$ & $\begin{array}{l}\text { Peak He } \\
\text { (ent/orb) }\end{array}$ & $\begin{array}{l}\text { Assoc. Flare } \\
\text { (Day/UT) }\end{array}$ & $\begin{array}{c}\operatorname{Imp} . \\
\text { X-гау/Ho }\end{array}$ & Location & $\begin{array}{l}\text { Region } \\
\text { Number }\end{array}$ \\
\hline 1 & - & $207.7 / 90$ & 208.0 & $209.5 / 90$ & - & - & - & - & - \\
\hline 2 & - & $213.0 / 90$ & 213.8 & $214.9 / 90$ & - & Jul30/0736 & $\mathrm{M} 4 / 2 \mathrm{~B}$ & N20E45 & 6180 \\
\hline 3 & 218 & $296.5 / 90$ & 296.7 & $297.6 / 90$ & 43 & - & - & - & - \\
\hline 4 & 451 & $26.0 / 91$ & 27.2 & $29.3 / 91$ & 201 & $\operatorname{Jan} 25 / 0630$ & $X 10 / S F$ & S16E78 & 6471 \\
\hline 5 & 462 & 31.3 & 31.7 & 32.4 & 481 & $\operatorname{Jan} 31 / 0230$ & $\mathrm{X} 1 / 2 \mathrm{~B}$ & S17W 35 & 6469 \\
\hline 6 & 481 & 39.3 & 39.6 & 39.9 & 53 & Febo7/1502 & $M 8 / 1 F$ & S10W 86 & 6471 \\
\hline 7 & 322 & 56.4 & 56.5 & 57.6 & 235 & Feb25/0819. & $X 1 / 2 N$ & S16W80 & 6497 \\
\hline 8 & 548 & 66.4 & 66.9 & 67.2 & 72 & Mat07/0748 & $X 5 / 3 B$ & S20E66 & 6538 \\
\hline 9 & 562 & 72.0 & 72.7 & 73.0 & 39 & $\operatorname{Mar12/1250}$ & $\mathrm{XI} / 2 \mathrm{~B}$ & SO7E59. & 6545 \\
\hline 10 & 587 & 82.1 & 83.1 & 84.6 & 9026 & $\operatorname{Mar} 22 / 2247$ & $x 9 / 3 B$ & $\mathrm{~S} 26 \mathrm{E} 28^{\circ}$ & 6555 \\
\hline 11 & 593 & 84.6 & 85.4 & 87.6 & 3234 & Mfar25/0818 & $x 5 / 3 B$ & $524 W_{13}$ & 6555 \\
\hline 12 & 613 & 93.0 & 93.6 & 34.5 & 60 & Apr02/2327 & $\mathrm{M} 6 / 3 \mathrm{~B}$ & N14W00. & .6562 \\
\hline 13 & 655 & 110.5 & 110.8 & 111.2 & 75 & Apr 20/08 $\equiv 2$ & $X_{1 / 3 N}$ & No8W50 & 6583 \\
\hline 14 & 684 & 122.1 & 122.6 & 123.1 & 46 & - & - & - & - \\
\hline 15 & 704 & 130.7 & 130.9 & 131.7 & 78 & - & - & - & - \\
\hline 16 & 710 & 133.1 & 133.4 & 135.0 & 517 & $\mathrm{May} 13 / 0144$ & $\mathrm{M8/-}$ & S09W90 & 6615 \\
\hline .17 & 730 & 139.0 & 141.5 & 142.9 & 63 & $\operatorname{May} 18 / 0546$ & $x 2 / 2 B$ & N 32 W 85 & 6619 \\
\hline 18 & 756 & 151.2 & 152.2 & 153.0 & 91 & Mfay $30 / 0938$ & $M 18 / 1 B$ & N07E30 & 6654 \\
\hline 19 & 774 & 153.0 & 159.6 & 162.1 & 23404 & Jun04/0352 & $\mathrm{X} 12 / 3 \mathrm{~B}$ & N30E70 & 6659 \\
\hline 20 & 781 & 162.1 & 162.6 & 165.5 & 13236 & Jun11/0209 & $\mathrm{X}_{12 / 3 \mathrm{~B}}$ & N31W 17 & 6659 \\
\hline 21 & 791 & 166.3 & 166.7 & 173.6 & 10101 & Jun $15 / .0821$ & $\times 12 / 3 B$ & N33W 69 & 6659 \\
\hline 22 & 829 & 179.5 & 183.0 & 188.3 & 1648 & Jun 28/0626 & $\mathrm{M} 6 /-$ & N30E85 & 6703 \\
\hline 23 & 843 & 188.4 & 189.1 & 190.3 & 370 & $\mathrm{Ju} 107 / 0223$ & $X_{1 / 2 B}$ & N26E03 & 6703 \\
\hline 24 & 942 & 231.1 & 231.5 & 231.9 & 45 & - & - & - & - \\
\hline 25 & 961 & 238.7 & 239.7 & 241.4 & 156 & Aug $25 / 0115$ & $\times 2 / 2 B$ & N25E64 & 6810 \\
\hline 26 & 1040 & 273.0 & 273.7 & 276.3 & 212 & Sep $29 / 1533$ & $A 17 / 4 B$ & S21E32 & 6853 \\
\hline
\end{tabular}

- Event 19 may associate three solar flares peaked at Jun?:1529 (X12/1E, N25E90), Jun4/0352 $(X 12 / 3 B, N 30 E 70)$, and $J u n 6 / 0112(X 12 / 4 B, N 33 E 44)$ in the same $30 A A$ region 6659 . The source for the flare identification (columns 7, 8, 9, and 10 ) is from Solar-Goophysical Data books (1991). 
Ejections. Even if there is some coverage outage, the existing cata can still be studied to give, at least, limits on the parameters. In some cases, it is necessary to sum several small events to fit an energy spectrum. In these cases it is assumed that the resulting spectrum applies to each of the erents within the sum.

The helium energy spectrum is fit to a power law:

$$
\frac{d J}{d E}=A\left(E / E_{0}\right)^{-\gamma}
$$

with $E_{0}=66 \mathrm{MeV} /$ nucleon and $A$ the amplitude and $\gamma$ the spectral index. The best estimates of $A$ and $\gamma$ are given in columns 2 and 3 of Table 3 . The total particle fluence is obtained by integrating over the duration of the event, and this is given, also, along with the event duration, in Table 3.

As we have shown previously, the Hydrogen spectra are different from the Helium spectra and the time profile of an SEP event may be different for Hydrogen and Helium. We have utilized GOES and IMP-8 data to determine the proton spectrum, fitting again to a power law such as equation (4). The proton amplitude, spectral index, total fluence and event duration are given in. columns 2-5 of Table 4. Comparison of Tables 3 and 4 show that the proton spectra are generally much harder than the Helium spectra and, as expected, the total proton fluence is several orders of magnitude larger than the Helium results.

We have developed a modeling technique for SEP events which assumes that the heavy ion spectra follow the Helium spectrum (not the proton spectrum) with a spectral amplitude determined by the relative coronal abundances of the different species, corrected for any heavy ion enhancement (or deficiency) in the event. To determine such an enrichment parameter, we look at the total heavy ions observed during the flare period by ONR-604. on CRRES If an enhanced abundance is observed, this is used to define a pseudo$\mathrm{Fe} / \mathrm{O}$ ratio which then gives the model parameter $\delta$. Both the $\mathrm{Fe} / \mathrm{O}$ and $\delta$ results are given in Table 4. If no heavy ions are observed, then the Fe/O ratio is set at its nominal value (0.155) and the $\delta$ parameter is 0 . For these events we assume normal solar energetic particle abundances.

It should be remarked that this analysis is at relatively high energy, i.e. 50100 's of $\mathrm{MeV} /$ nucleon, where statistics for power-law spectra are poor. Good measurements are obtained for only the largest flares, with tine smaller flares giving only limits. The range over which the model holds some validity is $\mathrm{E}>$ $10 \mathrm{MeV} /$ nucleon, the interval that is most important for the Single Event Upset problem in microelectronic circuitry. 
TABLE 3. He Spectra and Fluences

\begin{tabular}{|c|c|c|c|c|}
\hline $\begin{array}{l}\text { Event } \\
\text { Number }\end{array}$ & $\begin{array}{c}\text { Amplitude } \\
A^{-}\end{array}$ & $\begin{array}{c}\text { Index } \\
y\end{array}$ & $\begin{array}{l}\text { Fluence } \\
\text { (at } E_{0} \text { ) }\end{array}$ & $\begin{array}{c}\text { Duration } \\
\text { (Days) }\end{array}$ \\
\hline 1 & $0.071 \pm 0.008$ & $3.27 \pm 0.47$ & $1.1 \times 10^{4}$ & 1.8 \\
\hline 2 & $0.071 \pm 0.008$ & $3.27 \pm 0.47$ & $1.2 \times 10^{4}$ & 1.9 \\
\hline 3 & $0.071 \pm 0.008$ & $3.27 \pm 0.47$ & $6.7 \times 10^{3}$ & 1.1 \\
\hline 4 & $0.166 \pm 0.014$ & $2.22 \pm 0.39$ & $4.7 \times 10^{4}$ & 3.3 \\
\hline 5 & $0.139 \pm 0.010$ & $5.43 \pm 0.33$ & $1.3 \times 10^{4}$ & 1.1 \\
\hline 6 & $0.081 \pm 0.007$ & $3.21 \pm 0.39$ & $4.2 \times 10^{3}$ & 0.6 \\
\hline 7 & $0.108 \pm 0.006$ & $1.54 \pm 0.30$ & $1.1 \times 10^{4}$ & 1.2 \\
\hline 8 & $0.081 \pm 0.007$ & $3.21 \pm 0.39$ & $5.6 \times 10^{3}$ & 0.8 \\
\hline 9 & $0.081 \div 0.007$ & $3.21 \pm 0.39$ & $7.0 \times 10^{3}$ & 1.0 \\
\hline 10 & $15.82 \pm 0.44$ & $6.33 \pm 0.12$ & $3.4 \times 10^{6}$ & 2.5 \\
\hline 11 & $1.226 \div 0.026$ & $5.07 \pm 0.10$ & $3.2 \times 10^{5}$ & 3.0 \\
\hline 12 & $0.071 \doteq 0.008$ & $3.27 \pm 0.47$ & $9.2 \times 10^{3}$ & 1.5 \\
\hline 13 & $0.081 \pm 0.007$ & $3.21 \pm 0.39$ & $4.9 \times 10^{3}$ & 0.7 \\
\hline 14 & $0.081 \doteqdot 0.007$ & $3.21 \pm 0.39$ & $7.0 \times 10^{3}$ & 1.0 \\
\hline 15 & $0.081 \doteq 0.007$ & $3.21 \pm 0.39$ & $7.0 \times 10^{3}$ & 1.0 \\
\hline 16 & $0.236 \doteqdot 0.012$ & $3.31 \pm 0.24$ & $3.9 \times 10^{4}$ & 1.9 \\
\hline 17 & $0.071 \doteq 0.008$ & $3.27 \pm 0.47$ & $2.4 \times 10^{4}$ & 3.9 \\
\hline 18 & $0.071=0.008$ & $3.27 \pm 0.47$ & $1.1 \times 10^{4}$ & 1.8 \\
\hline 19 & $10.98 \pm 0.21$ & $4.10 \pm 0.09$ & $8.6 \times 10^{6}$ & 9.1 \\
\hline 20 & $34.18 \pm 1.28$ & $4.31 \pm 0.17$ & $1.0 \times 10^{7}$ & 3.4 \\
\hline 21 & $4.16 \div 0.15$ & $4.28 \pm 0.16$ & $2.6 \times 10^{5}$ & 7.3 \\
\hline 22 & $0.422 \div 0.014$ & $4.53 \pm 0.15$ & $3.2 \times 10^{5}$ & 8.8 \\
\hline 23 & $0.160 \div 0.010$ & $5.42 \pm 0.28$ & $2.6 \times 10^{4}$ & 1.9 \\
\hline 24 & $0.081=0.007$ & $3.21 \pm 0.39$ & $5.6 \times 10^{3}$ & 0.8 \\
\hline 25 & $0.065 \pm 0.003$ & $3.24 \div 0.29$ & $1.5 \times 10^{4}$ & 2.7 \\
\hline $2 \hat{0}$ & $0.158 \pm 0.017$ & $4.06 \pm 0.46$ & $4.5 \times 10^{4}$ & 3.3 \\
\hline
\end{tabular}

* in particles $/ \mathrm{m}^{2}-\mathrm{sr}-\mathrm{s}-\mathrm{MeV} / \mathrm{nucleon} ;$ * in partieles $/ \mathrm{m}^{2}-\mathrm{sr}-\mathrm{MleV} / \mathrm{nucleor}$. 
TABLE 4. Proton Spectra and SEP Model Parameters

\begin{tabular}{|c|c|c|c|c|c|c|}
\hline Event & $\begin{array}{l}\text { Amplitude } \\
A_{p}^{-}\end{array}$ & $\begin{array}{l}\text { Index } \\
\gamma_{p}\end{array}$ & $\begin{array}{l}\text { Fluence } \\
\text { (at } E_{0} \text { ) }\end{array}$ & $\begin{array}{c}\text { Duration } \\
\text { (Days) }\end{array}$ & $\mathrm{Fe} / \mathrm{O}$ & $\delta$ \\
\hline 1 & $111 \pm 8$ & $2.72 \pm 0.05$ & $2.88 \times 10^{7}$ & 3 & 0.155 & 0.000 \\
\hline 2 & $202 \pm 52$ & $3.39 \pm 0.18$ & $\therefore 3.49 \times 10^{7}$ & 2 & 0.155 & 0.000 \\
\hline 3 & $37 \pm 8$ & $1.94 \pm 0.15$ & $6.39 \times 10^{6}$ & 2 & 0.155 & 0.000 \\
\hline 4 & $53 \pm 9$ & $2.10 \pm 0.12$ & $1.83 \times 10^{7}$ & 4 & 0.306 & 0.038 \\
\hline 5 & $129 \pm 43$ & $3.38 \pm 0.24$ & $2.23 \times 10^{7}$ & 2 & 1.030 & 0.105 \\
\hline 6 & $56 \pm 13$ & $2.38 \pm 0.21$ & $4.84 \times 10^{6}$ & 1 & 1.030 & 0.105 \\
\hline 7 & $56 \pm 8$ & $2.23 \pm 0.11$ & $9.68 \times 10^{6}$ & 2 & 0.225 & 0.021 \\
\hline 8 & $33 \pm 5$ & $1.48 \pm 0.11$ & $2.85 \times 10^{6}$ & 1 & 1.030 & 0.105 \\
\hline 9 & $75 \pm 13$ & $2.40 \div 0.12$ & $6.48 \times 10^{6}$ & 1 & 1.030 & 0.105 \\
\hline 10 & $20040 \pm 7001$ & $4.64 \pm 0.40$ & $3.46 \times 10^{9}$ & 2 & $0.9 \div 6$. & 0.101 \\
\hline 11 & $579 \pm 115$ & $4.16 \pm 0.23$ & $2.00 \times 10^{5}$ & 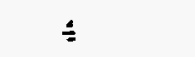 & $3.2 \div 3$ & 0.196 \\
\hline 12 & $91 \pm 11$ & $2.70 \pm 0.08$ & $1.57 \times 10^{7}$ & 2 & 0.155 & 0.000 \\
\hline 13 & $31 \pm 5$ & $1.45 \pm 0.11$ & $2.68 \times 10^{6}$ & 1 & 1.030 & 0.105 \\
\hline 14 & $30 \div 4$ & $1.40 \pm 0.09$ & $2.59 \times 10^{6}$ & 1 & 1.030 & 0.105 \\
\hline 15 & $50 \pm 4$ & $1.97 \div 0.05$ & $4.32 \times 10^{6}$ & 1 & 1.030 & 0.105 \\
\hline 16 & $445 \pm 66$ & $3.08 \pm 0.13$ & $7.69 \times 10^{\top}$ & 2 & $6.20 \mathrm{~S}$ & 0.205 \\
\hline 17 & $71 \pm 6$ & $2.25 \div 0.06$ & $2.45 \times 10^{7}$ & 4 & 0.155 & 0.000 \\
\hline 18 & $83 \div 12$ & $2.74 \pm 0.11$ & $1.43 \times 10^{7}$ & 2 & 0.135 & 0.000 \\
\hline 19 & $876 \pm 140$ & $3.08 \pm 0.19$ & $6.81 \times 10^{3}$ & 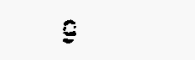 & 0.182 & -0.023 \\
\hline 20 & $3238 \pm 917$ & $2.90 \pm 0.26$ & $1.12 \times 10^{9}$ & 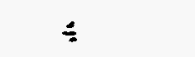 & $0.05=$ & -0.054 \\
\hline 21 & $1261 \pm 307$ & $2.76 \pm 0.22$ & $8.72 \times 10^{8}$ & $\varepsilon$ & $0 . \leqslant 5 ?$ & 0.064 \\
\hline 22 & $153 \pm 23$ & $2.84 \pm 0.11$ & $1.19 \times 10^{8}$ & $\mathrm{~g}$ & 0.155 & 0.000 \\
\hline 23 & $306 \pm 119$ & $4.10 \pm 0.23$ & $7.93 \times 10^{7}$ & 3 & 1.030 & 0.105 \\
\hline 24 & $24 \pm 4$ & $1.38 \pm 0.11$ & $2.07 \times 10^{6}$ & 1 & 1.030 & 0.105 \\
\hline 25 & $13 \cdot 1 \div 34$ & $3.26 \pm 0.18$ & $4.53 \times 10^{i}$ & $\leq$ & 0.153 & 0.000 \\
\hline 20 & $71 \pm 10$ & $2.45 \pm 0.10$ & $2.45 \times 10^{i}$ & $\div$ & 9.335 & 0.228 \\
\hline
\end{tabular}

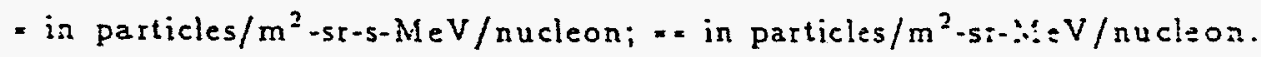


We can utilize the resuits of Tables $2-4$, within the uncertainties, to look at the probable behavior of the 26 flares that have been studied. Utilizing the model, we can predict the heavy ion spectra that would be observed with a larger instrument at the energies under consideration here. These results are shown in Figure 21. The left-hand plots show the measured $\mathrm{H}$ (solid) and $\mathrm{He}$ (dashed) energy spectra while the right-hand graphs show the inferred heavy ion abundance at $-100 \mathrm{MeV} /$ nucleon. In the model all spectra are power laws, and the Helium spectra index is used for all heavier elements. The relative importance of the heavy ions can be determined by looking at the ratio between helium and, for example, the CNO peak, considering also the steepness of the helium energy spectrum. A glance through Figure 21 shows the variability in the set of SEP events that were encountered, and measured by ONR-604, during the CRRES mission.

\section{Geomagnetic Transmission}

For Earth - orbiting satellites at low altitude, the Earth's magnetic field and the solid Earth itself provide significant additional protection from the interplanetary heavy ion environment. While the physics of this shielding effect is simply the Lorentz force on a moving charge, an exact calculation is complicated by the complex particle trajectories which can result, especially in the transition region where the shielding effect becomes important. The Earth's magnetic field produces an east-west asymmetry in the flux arriving at some observing location (magnetic radius and latitude; $r, \lambda$ ) such that for a dipole magnetic field, particles with a specific momentum per unit charge (rigidity, $\rho=p / Z$ ) only arrive from a cone of directions surrounding the. west (for positive charges) with an angular extent, $\omega$, given by

$$
\cos (\omega)=(2 Y / \cos (\lambda))-Y^{2} \cos (\lambda)
$$

where $Y^{2}=M /\left(\mathrm{cpr}^{2}\right), M$ is the magnetic dipole moment of the Earth, and $c$ is the . speed of light. The solid angle corresponding to this cone is equal to $2 \pi$ $(1+\cos (\omega))$.

To account for the shielding due to the solid Earth, the access cone solid angle is reduced by the fraction obscured by the Earth at alti-itie $h$ to obtain a total solid angle factor (Wilson et al., 1991):

$$
\Omega=\pi(1+\cos (\omega))\left\{1+\cos \left(\arcsin \left(R_{e} /\left[R_{e}+h i\right)\right\}\right.\right.
$$

The vertical cutoff rigidity approximation often employed to estimate the geomagnetic shielding effect is a simplification of this procesiure. It 


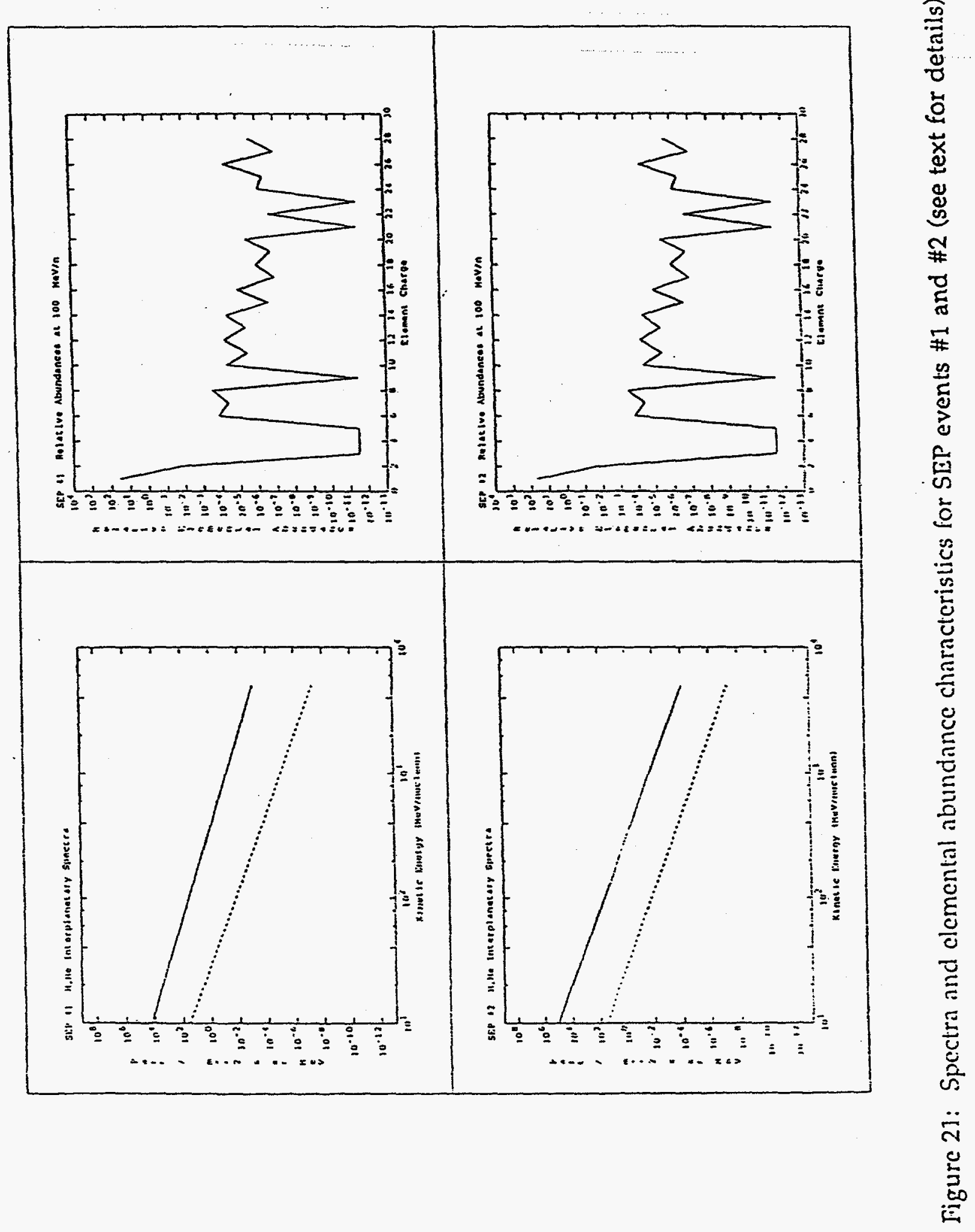




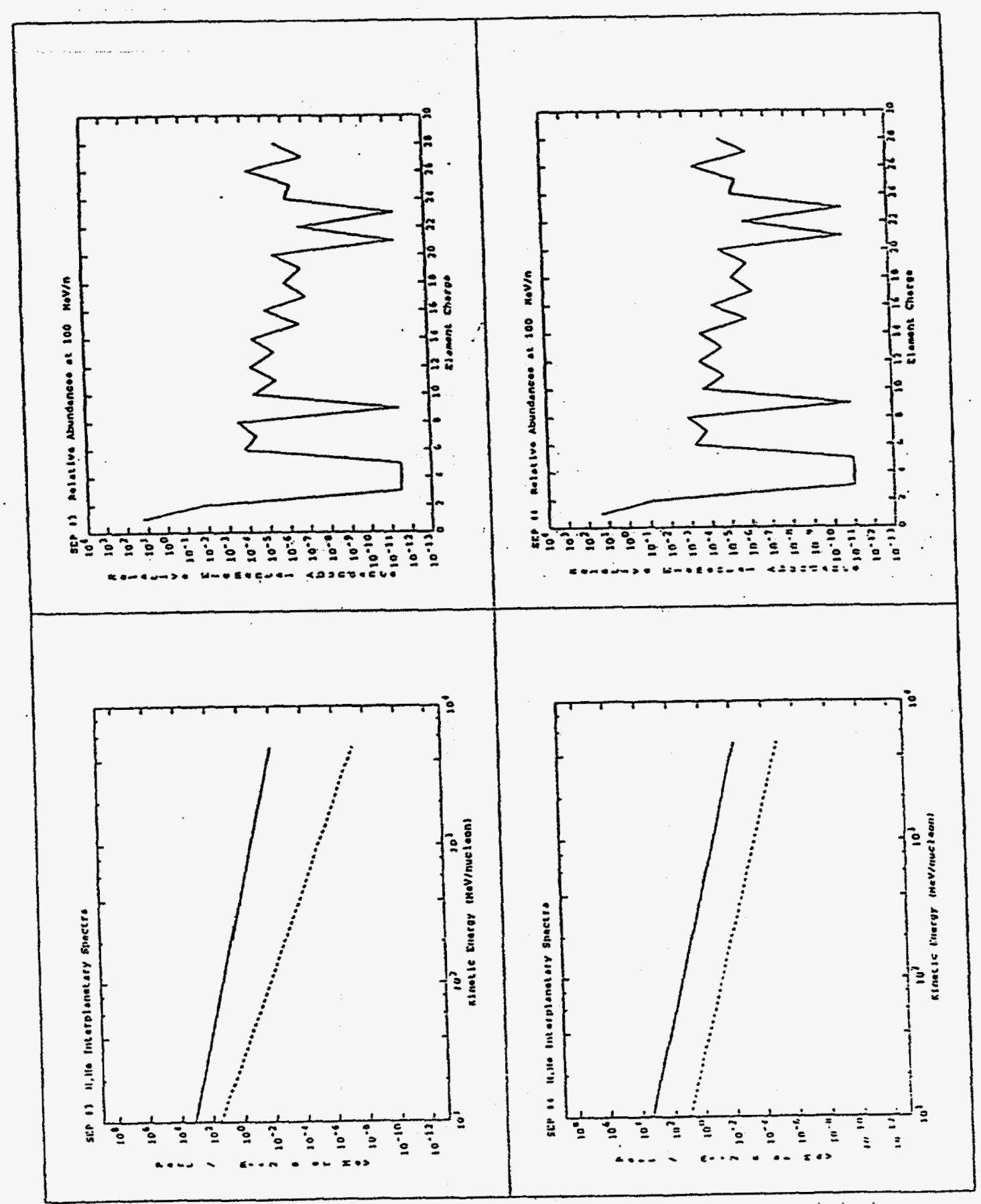

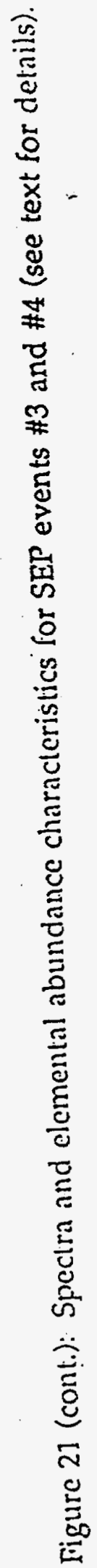




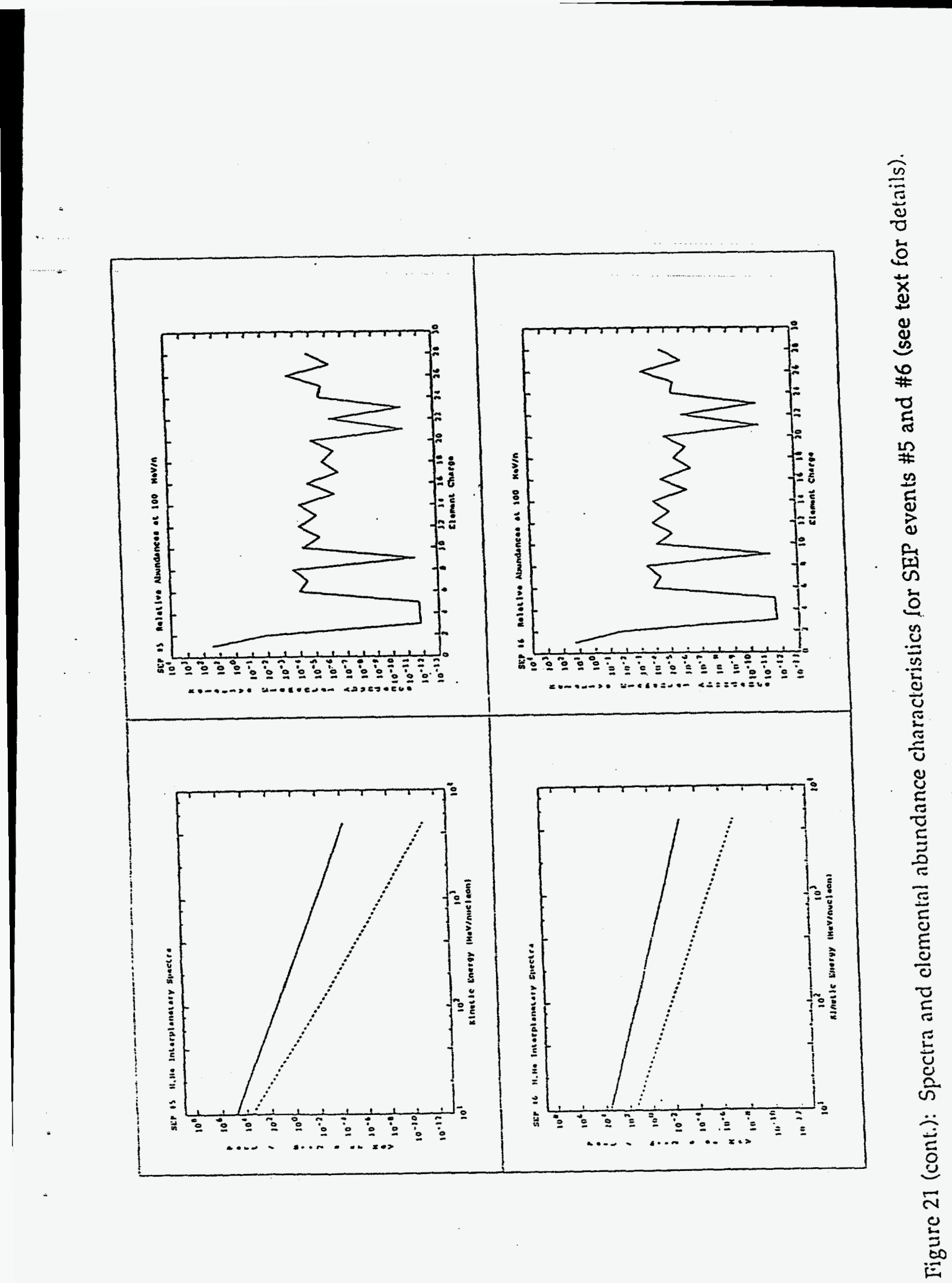




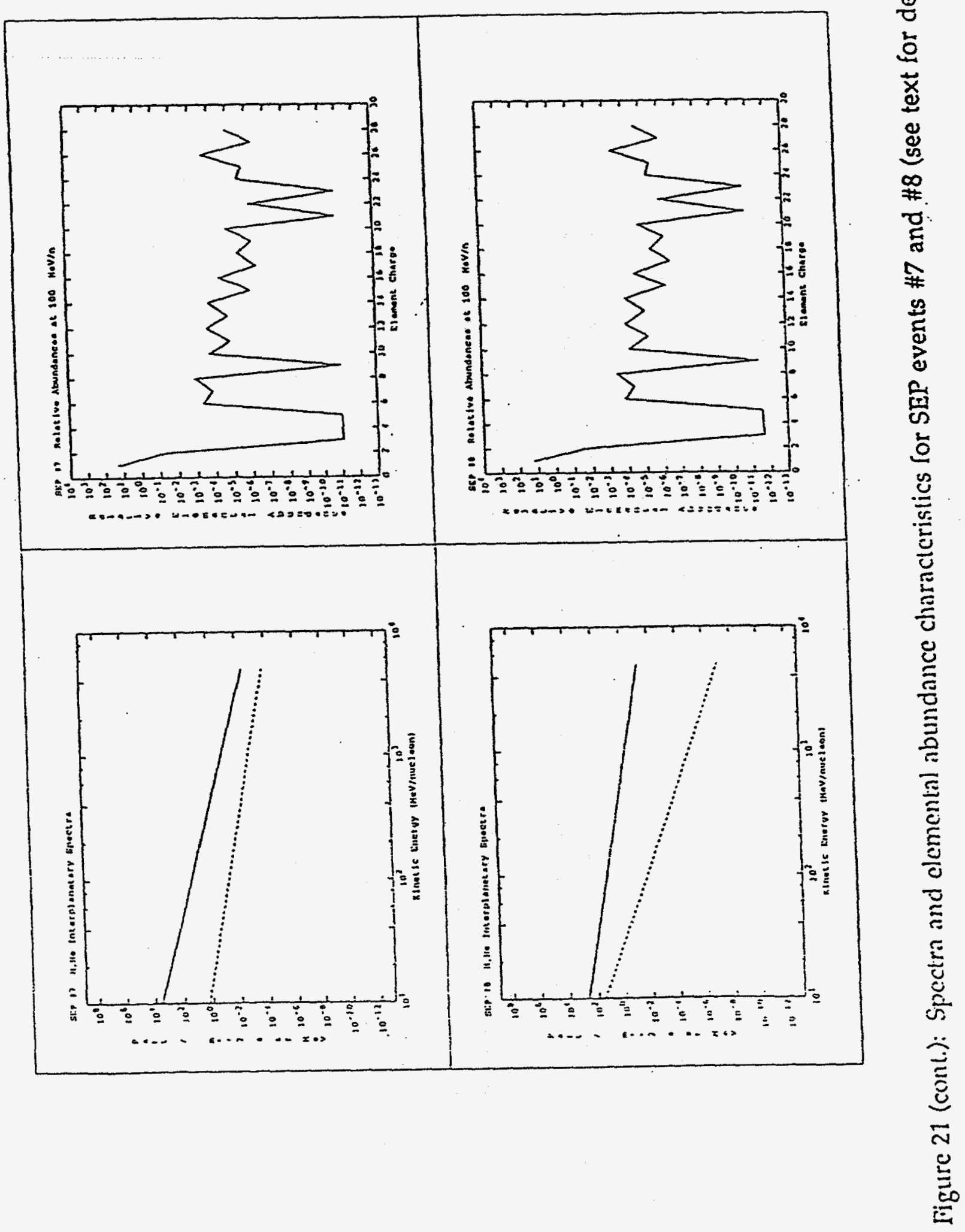




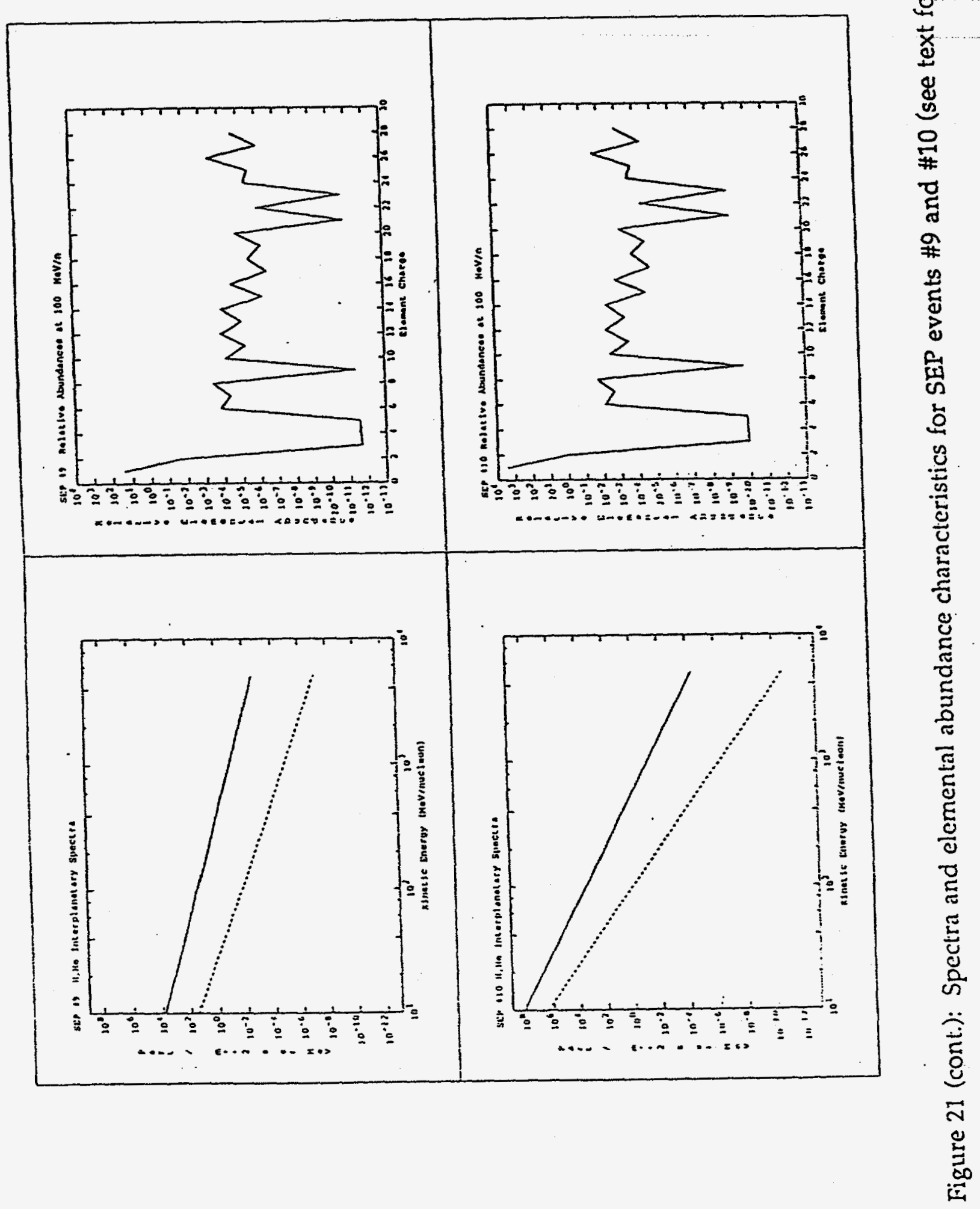



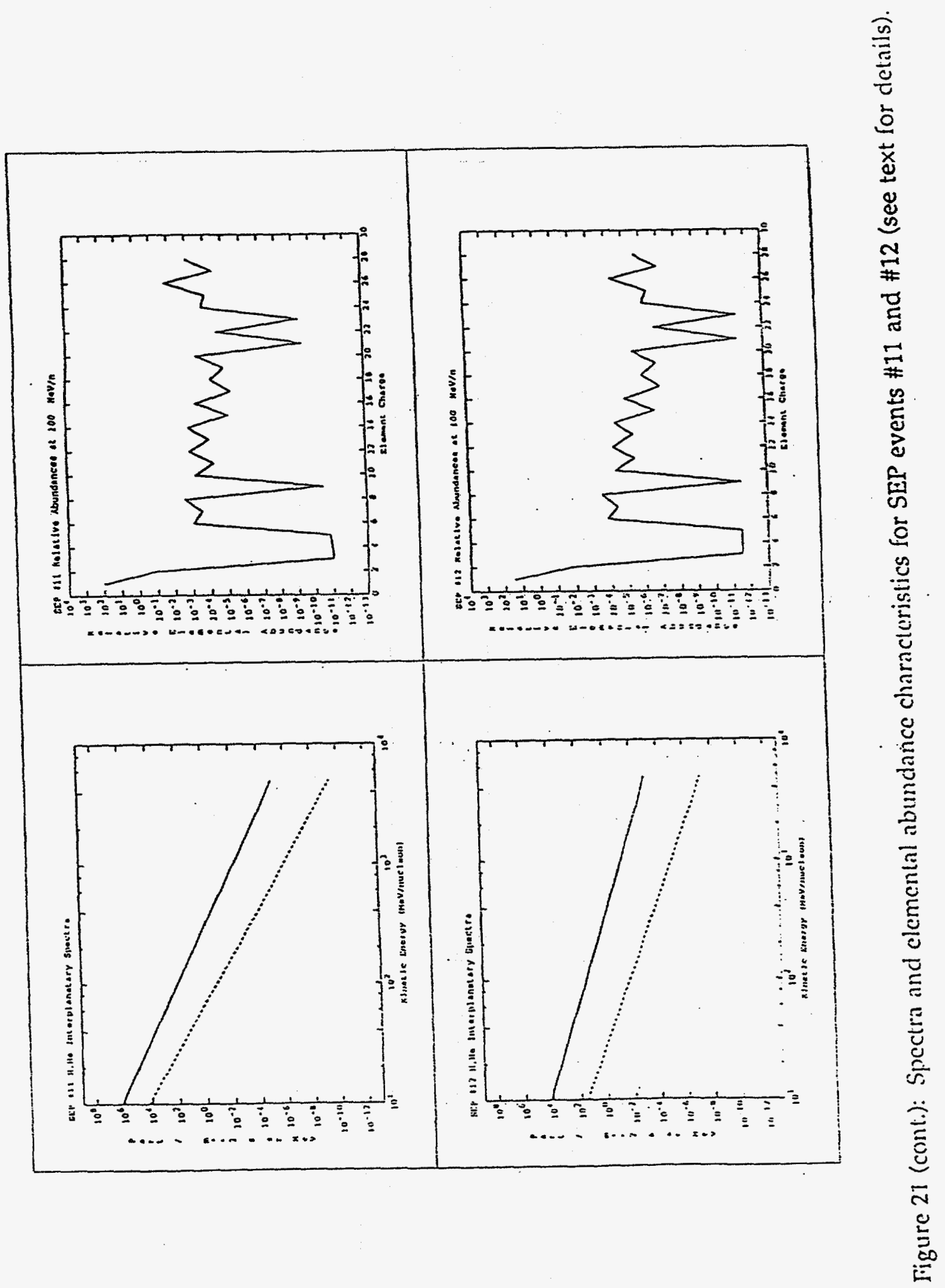


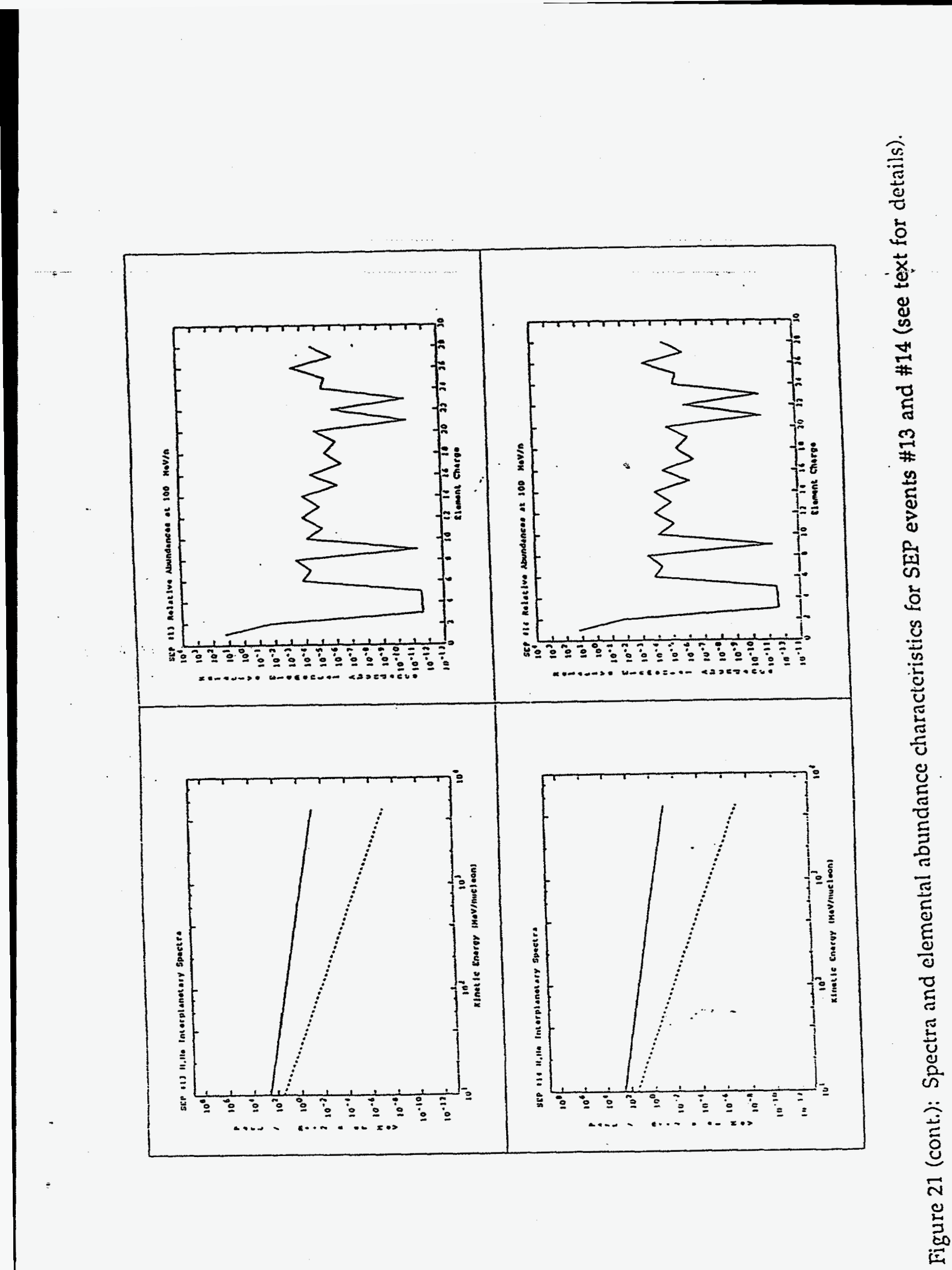




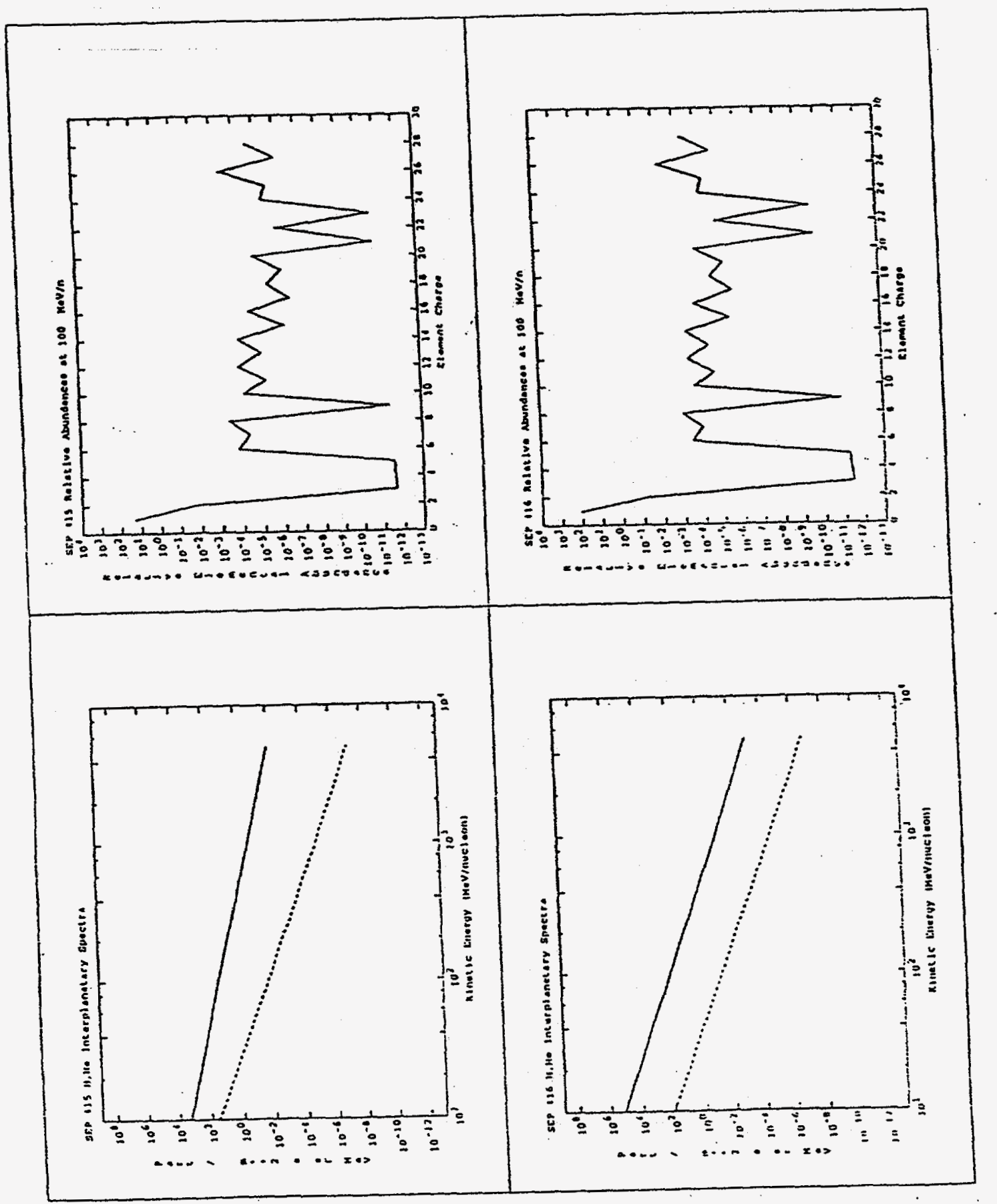

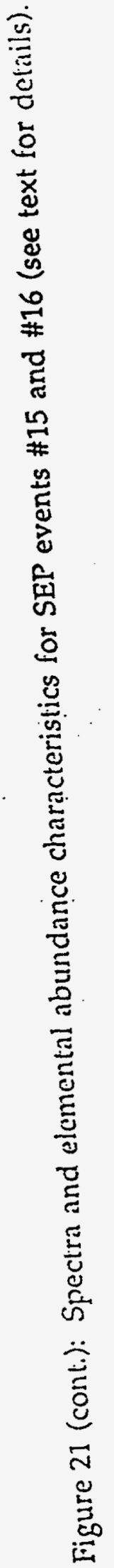




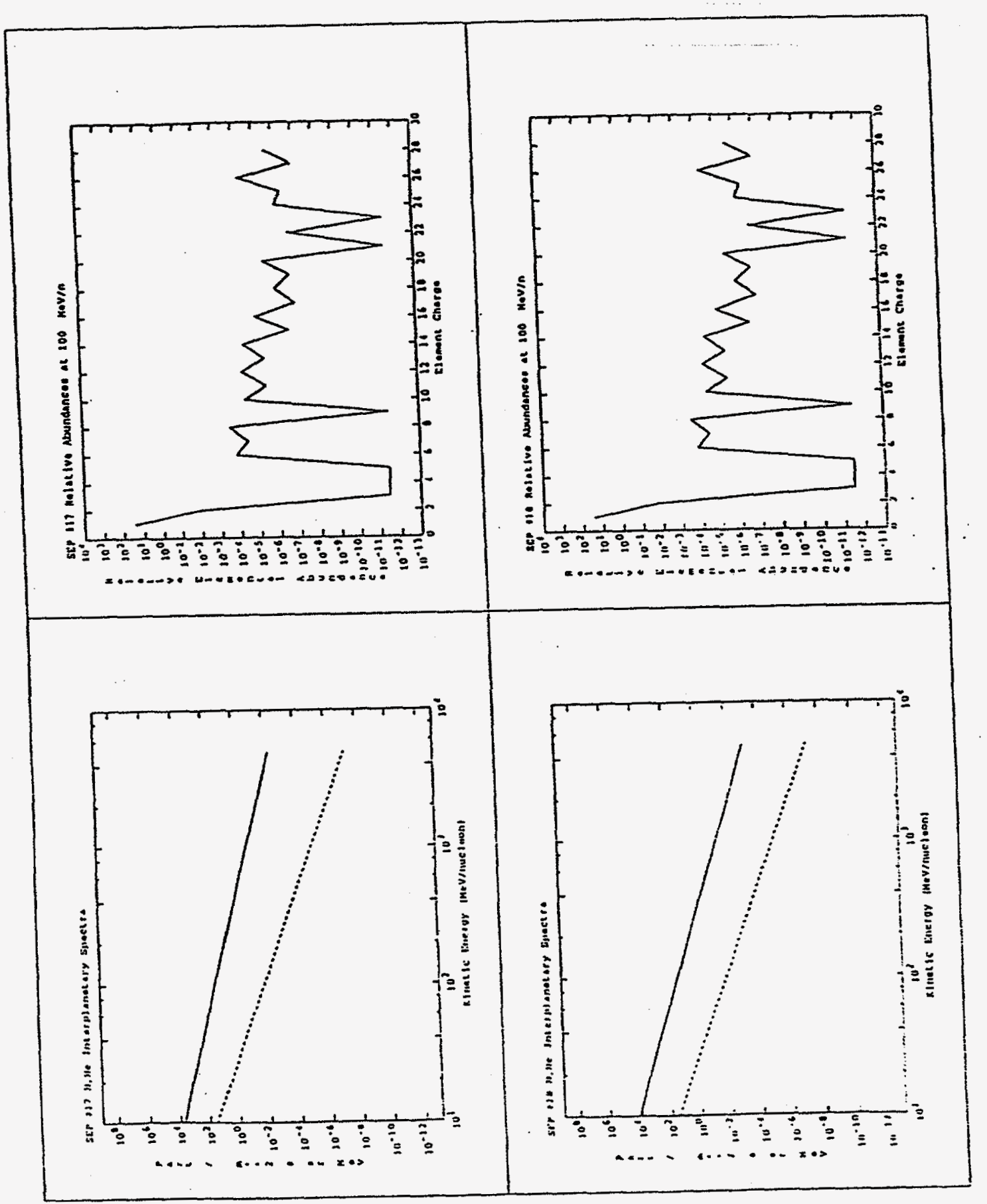

$\frac{\dot{\sigma}}{\frac{3}{0}}$

는

获

\&

$\infty$

号

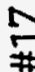

疍

$\stackrel{0}{\circ}$

它

능

告

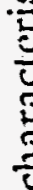

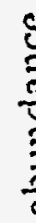

亲

잉

ह气

芒

त

些 


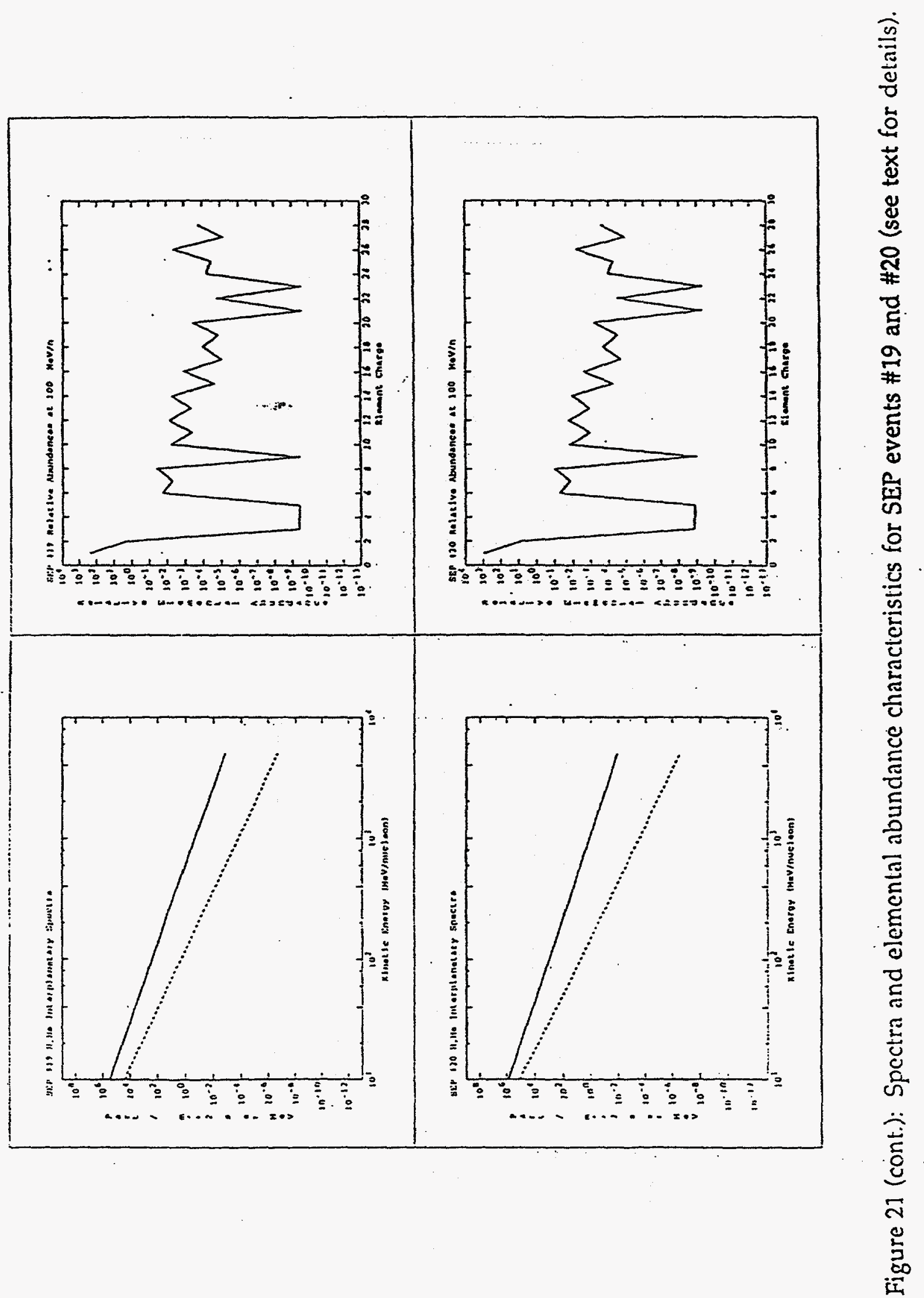




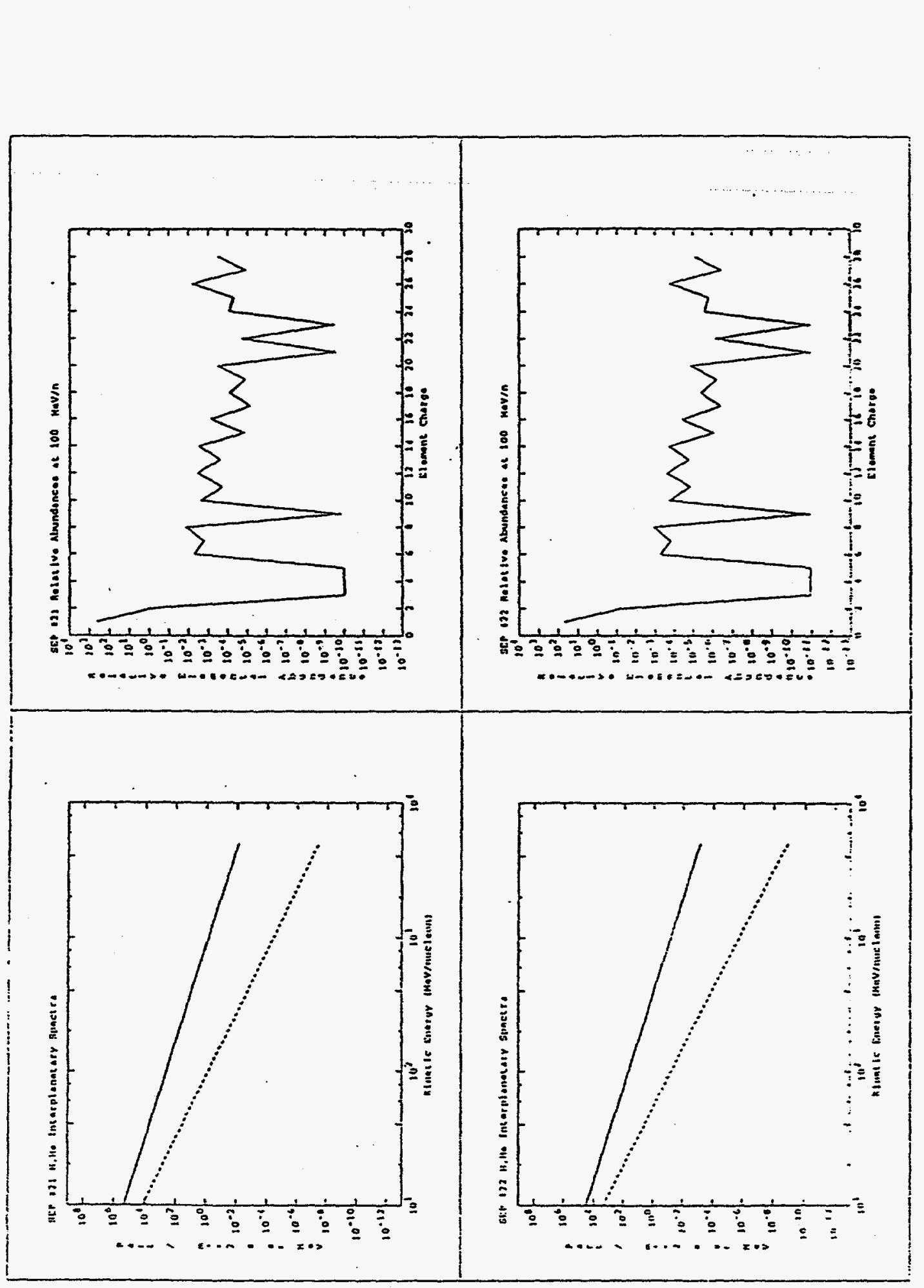

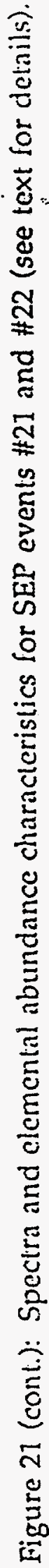




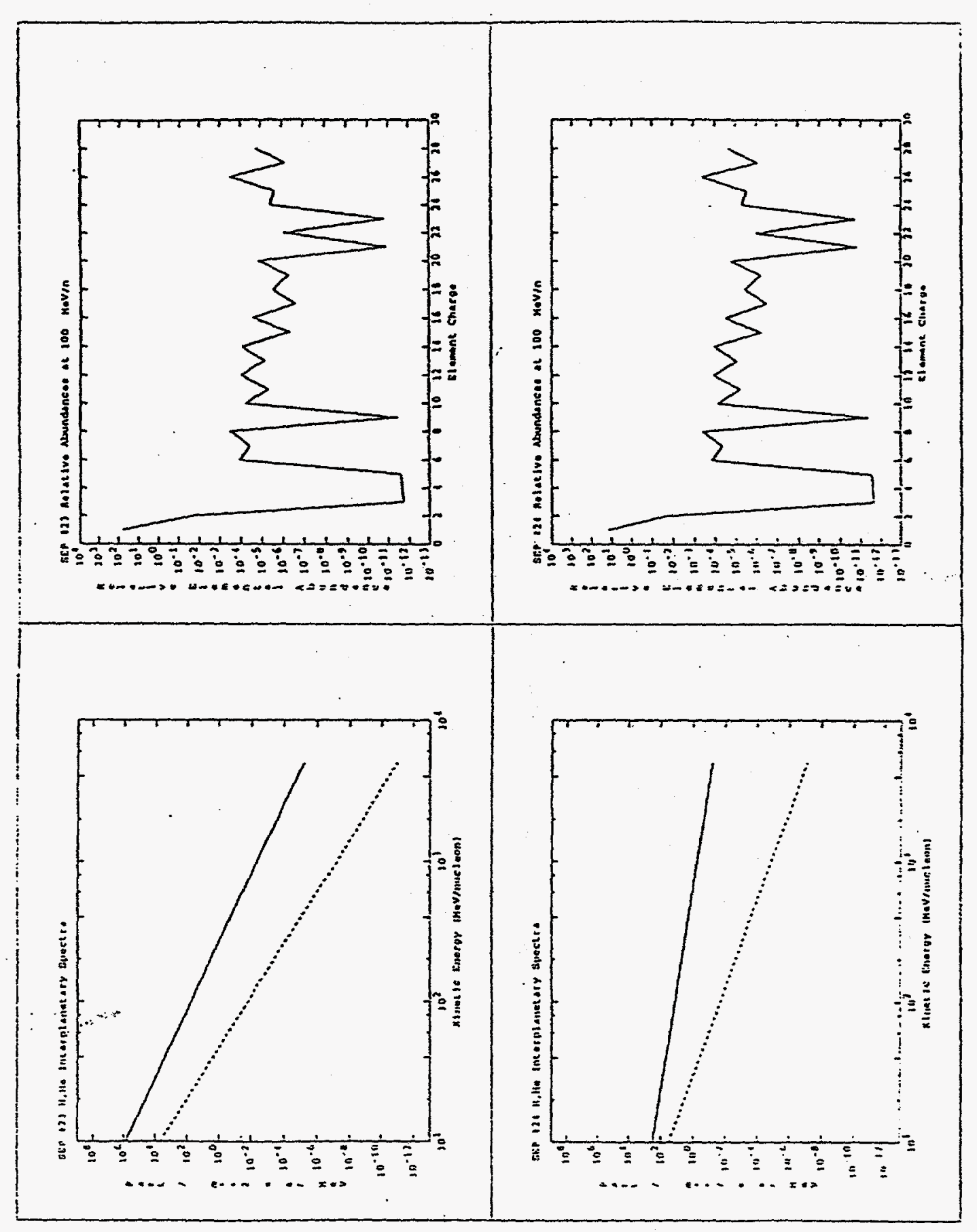

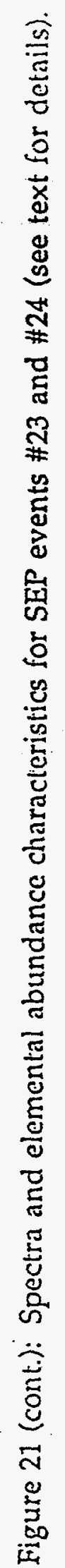




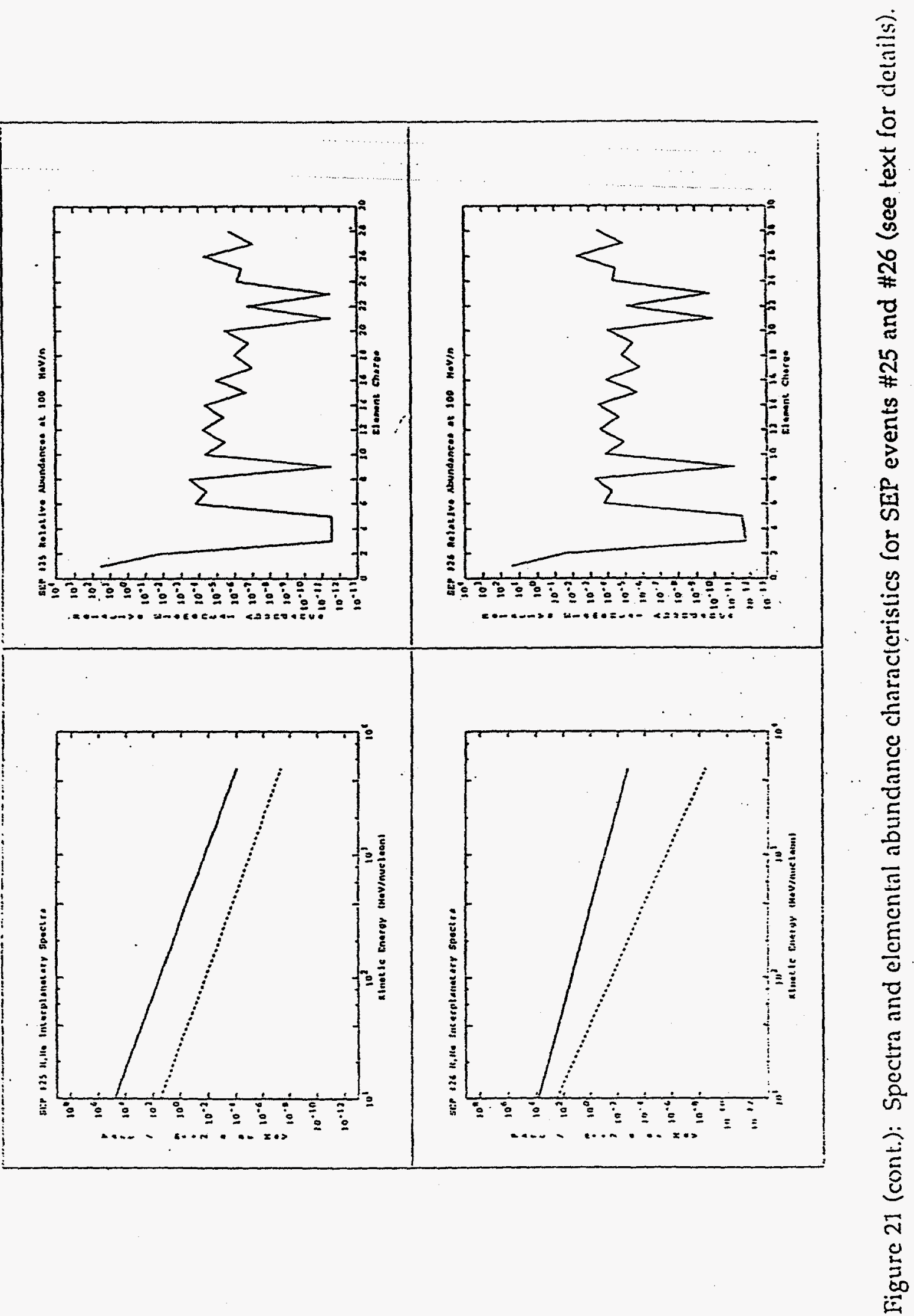


approximates the access function as a step function at the rigidity corresponding to a cone angle of $90^{\circ}$.

The GEOMAG Transmission function in CHIME calculates this access solid angle as a function of the ion energy and applies this filter function to the interplanetary heavy ion flux calculated by the procedures described in the previous sections. The user may select a location, an orbit, or a series of orbits over some period of time. When an orbit is specified the access filter is calculated as a time-weighted average. The transmission filter is applied separately to the anomalous component, which is treated as singly charged $(\mathrm{Z}=1)$ in this part of the calculation.

\section{The Linear Energy Transfer Spectrum Calculator}

The LET spectrum calculator in CHIME employs an integral method and a two-part (shield and target) spherical geometry model. The thicknesses of the shield and the target may be specified by the user in column density units $\left(\mathrm{mg} / \mathrm{cm}^{2}\right)$. Since the range depends on the density of the target and only weakly on the target composition, this approach permits the model to be used with different materials. Working from the sensitive region out, and for each ion species, minimum and maximum incident ion energies are calculated corresponding to pre-defined LET thresholds. At each threshold LET, the integral LET spectrum is calculated by integrating the heavy ion flux spectrum over this energy range and summing over all particle species.

In this implementation, the ion LET in the target and the ion energy outside the shield are both calculated using differences of the range function, $R(E, Z, M)$, for an ion with kinetic energy $E$, atomic number $Z$, and mass $M$. The range function is scaled at ion energy $E / M$ by $M Z^{-2}$ from the range tabulation for protons in silicon of Janni (1982). It does not depend on the charge state of the ion before it strikes the shield or target. A range extension is applied to account for the ion charge state at low velocities within the material based on the results of Heckman et al. (1960). One advantage of this method is that it fully accounts for the slowing-down of the incident ions within the target, including the lowenergy ions which stop in the target. Such ions have ranges less than the target thickness after penetrating the shield. The effects of nuclear interactions are not included in this model. For thick shields, when the effects of those interactions become important, this LET spectrum will over-estimate the real spectrum at high LET and underestimate the real spectrum at low LET. 


\section{THE CLEMENTINE RADIATION ENVIRONMENT}

As a joint program of the Department of Defense and NASA, the Clementine series of missions is designed to provide both deep space tests of miniaturized sensors, spacecraft and subsystems and new scientific information of the Moon and other objects in the near-Earth environment. Part of the deep space test effort is to determine the effect of the space radiation environment on the Clementine sensors and components. In support of this effort LSU has used the CHIME model, described in the previous section, to estimate the characteristics of the radiation environment to be encountered during the CLEMENTINE I mission.

\section{A. The Mission Parameters}

The CLEMENTINE I mission is composed of three distinct phases or components: 1) the Interstage Adapter (ISA) in a highly eccentric Earth orbit; 2) the spacecraft during Lunar Survey; and 3) the spacecraft during asteroid encounter. These phases are illustrated in Figure 22 which provides the time intervals as well as orbital parameters used in this study.

The ISA was used to boost the spacecraft toward the Moon and was left in a highly eccentric Earth orbit. An instrument package consisting of several different types of dosimeters and radiation monitors was mounted on the ISA to provide additional space radiation environment experimenis with minimal impact to the primary CLEMENTINE I mission. Figure 22 indicates that the characteristic ISA will have a perigee of $-350 \mathrm{~km}$, an apogee of $-167,000 \mathrm{~km}$, an orbital inclination of $67^{\circ}$, and a lifetime covering at least the nominal mission from 2/1/94 to $10 / 1 / 94$. This orbit will take the ISA deep into the Earth's magnetosphere where it will encounter a sizable dose from tie Earth's trapped radiation belts. The radiation environment in these belts is usually studied with the aid of available, "standard" models such as AP8 and AE8, and it is unlikely that our investigation could significantly contribute to such studies. Instead, we will concentrate on the interplanetary radiation environment and its penetration to the ISA orbit.

After lunar insertion, roughly three weeks after launch, the spacecraft will either be outside the geomagnetosphere or at very high $L$ vaiues for most of its lifetime. Thus, the geomagnetic transmission will have little effect on the spacecraft radiation environment. Here the most importar: effects will be the solar modulation variations over the mission lifetime anc :ie contributions from SEP events. The time periods used for the two CLENE IIINE I spacecraft phases are $2 / 21 / 94$ to $5 / 3 / 94$ for the Lunar Orbit phase and $5 ; 2 / 94$ to $8 / 31 / 94$ for the asteroid (Geographos) flyby phase. 


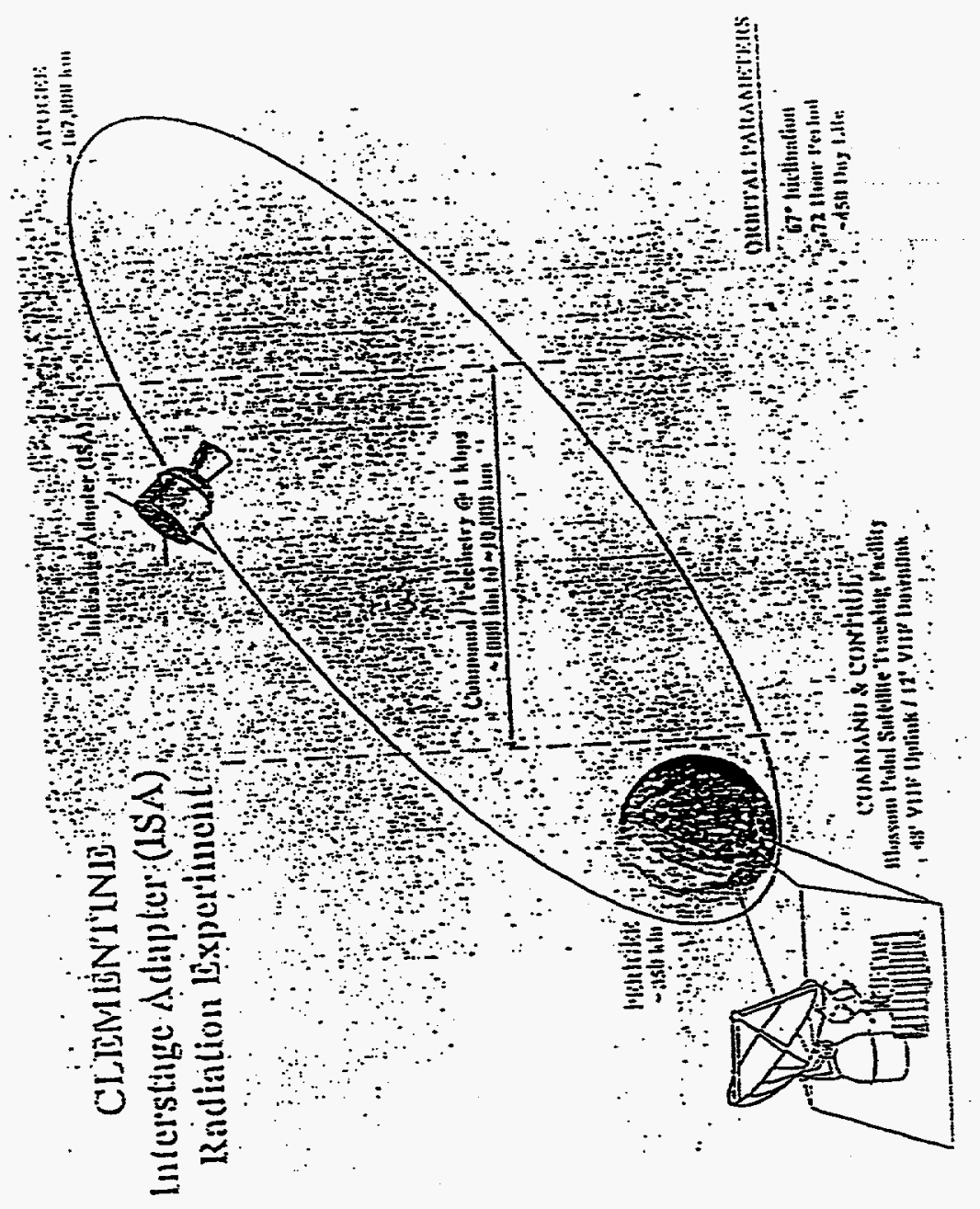

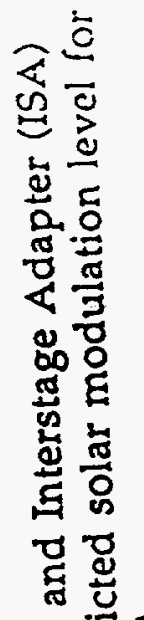

봃

总。

骂要

연

包高

E

오온

可

ฆ ย

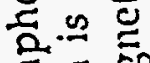

논

过

도을
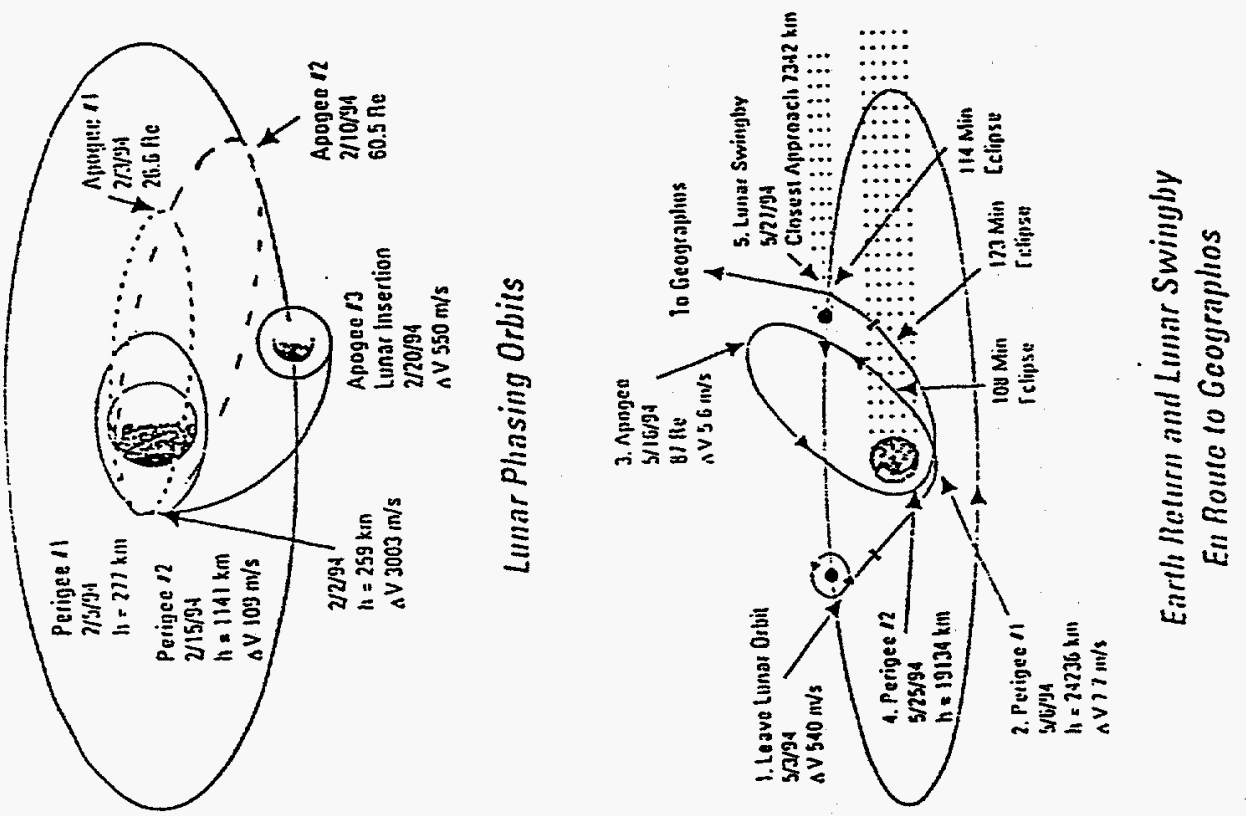

응

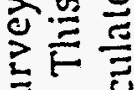

ज.

光

$\Xi$ ED

흥

吉范

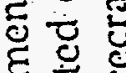

它芯

产兰

$\ddot{~}$

$\stackrel{2}{E}$ 


\section{B. Expected Solar Modulation Levels during the Mission}

The CHIME model was used to estimate the solar moduiation level (ọ) during the CLEMENTINE I mission. This level can vary from: $\rho-1500 \mathrm{MV}$ for solar maximum to about $\phi \sim 475 \mathrm{MV}$ for solar minimum. The $\rho$ values predicted for each month of 1994, and rounded to the nearest $10 \mathrm{MV}$, are shown in Table 5. A steady decrease in the modulation level is expected throughout the year as solar minimum levels are approached during late 1994 or early 1995. This implies that GCR and $A C$ radiation levels will increase during the mission. The average $\phi$ for the three mission phases are: $\phi \sim 588 \mathrm{MV}$ for the Lunar Orbit, $\phi$ $\sim 558 \mathrm{MV}$ for the asteroid flyby, and $\phi \sim 570 \mathrm{MV}$ for the full time period from $2 / 94$ to $10 / 94$.

TABLE 5 - Predicted Values of $\phi$ During 1994

\begin{tabular}{|ll|ll|}
\hline Month & $\begin{array}{l}\phi \\
\text { (MV) }\end{array}$ & Month & $\begin{array}{l}\phi \\
\text { (MV) }\end{array}$ \\
\hline January & 610 & July & 560 \\
February & 600 & August & 550 \\
March & 590 & September & 540 \\
April & 580 & October & 530 \\
May & 570 & November & 520 \\
June & 560 & December & 520 \\
\hline
\end{tabular}

\section{GCR Spectrum Variation}

The effect of solar modulation during 1994 on the GCR Fielium energy spectrum is illustrated in Figure 23 and is representative of the effects seen for energetic heavy ions. In panel A of the figure, the differentiai energy spectrum for January, 1994 and December, 1994 is shown. The peak flux of about $0.25-0.3$ (m²-sr-s-MeV/nucleon)-1 occurs at about $200-300 \mathrm{MeV} /$ nucleori and drops off by about an order of magnitude as the energy decreases to $10 \mathrm{MfeV} /$ nucleon. Since the solar modulation level decreases over the course of the year, the December flux will be larger than that at the start of the year. Notice also that the modulation effects are more pronounced at low energy ( $<I \mathrm{GeV} /$ nucleon). This can be more clearly seen in panel $B$ of the figure, which piots the ratio between the energy spectrum modulated for each month of 1 January, 94 spectrum. This figure illustrates the variation of $: \dot{:} \geq$ GCR flux as a function of both time and energy. Over the course of the yea:, the Helium flux at high energy ( $>1 \mathrm{GeV} /$ nucleon) should increase by about $10 \%$, while at low energy $(\sim 100 \mathrm{MeV} /$ nucleon$)$ the increase may be $30 \%$ to $35 \%$. 

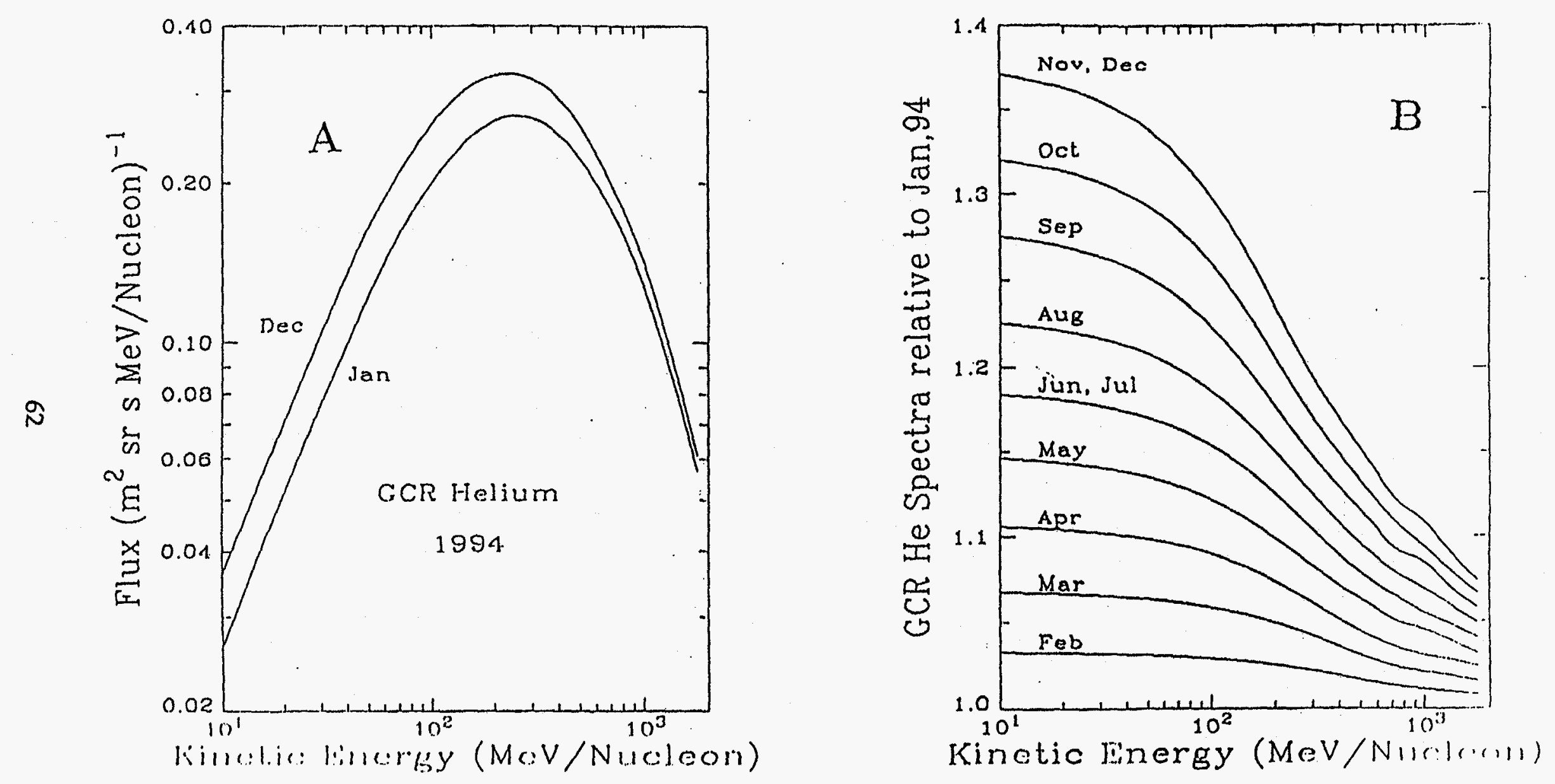

Figure 23: The effect of solar modulation during 1994 on the Galactic Cosmic Ray Helium spectrum. The Helium differential energy spectrum predicted for January, 1994 and December, 1994 is shown in panel $A$. The variation in the spectrum for each month during 1994, relative to January, is shown in panel $B$. 


\section{Anomalous Component Variation}

The equivalent variation of the anomalous Helium component during 1994 is shown in Figure 24. The energy spectra for January and December are in panel $A$ while the monthly variation is indicated in panel $B$. For energies greater than about $90-100 \mathrm{MeV} /$ nucleon the anomalous component flux is negligible compared to the GCR, but below about $30 \mathrm{MeV} /$ nucleon the $A C$ dominates the particle spectrum. In addition, the $A C$ is much more variable than the GCR during 1994 and is expected to increase by up to a factor of 3 between January and December.

\section{Expected SEP Events during Mission}

The CHIME program includes 26 SEP events which occurred during the CRRES mission, and which are characterized in Tables 2 to 4, as well as incorporating the proton fluence model of Feynman et al. (1993). The scope of the program, however, was directed toward support of the CRRES/SPACERAD mission and a description of the actual SEP events observed was sufficient. Therefore, CHIME does not attempt to predict the number or characteristics of SEP events for any future year. For this study, we have provided such a prediction for 1994 by examining the historical record and constructing "sample" SEP populations from the 1991 events.

\section{Historical Variation}

To make a reasonable prediction for the number and type of SEP events that may be seen during the CLEMENTINE I mission we need first to look at the historical SEP frequency and fluence variations. Previously, we saw a good correlation between the yearly averaged sunspot number per month and the number of SEP events per year observed by the GOES and IMP-8 spacecraft during the time period 1975 to 1993 (c.f. Figure 8). These data cover almost a full solar cycle, and the roughly linear correlation is plotted in pariel A of Figure 25. (Note that the y scale in this plot would need to be multiplied by a factor of 12 to obtain the mean yearly sunspot number.) A simple linear fit to this relation can then be applied to the sunspot number and used to generate an expected yearly SEP number. This is shown in panel $B$, where the observed mean monthly sunspot number for the years 1950 to 1993, with predicted values for 1994 and 1995 , is shown as the solid line and the predicted SEP number as the dashed curve. A comparison of the observed (solid curve) and inferred (dashed curve) SEP number is shown in panel C. While there are some diffe-ences in details, the general agreement between the two curves is quite good.

In panel D of Figure 25, the inferred SEP number is used to examine the event time frequency. Solar maximum peaks occurred during 1957, 1968, 1979 and 1989 and these are indicated on the panel as the inverted illed triangles. The CLEMENTINE I mission will take place during 1994, five years after the last 


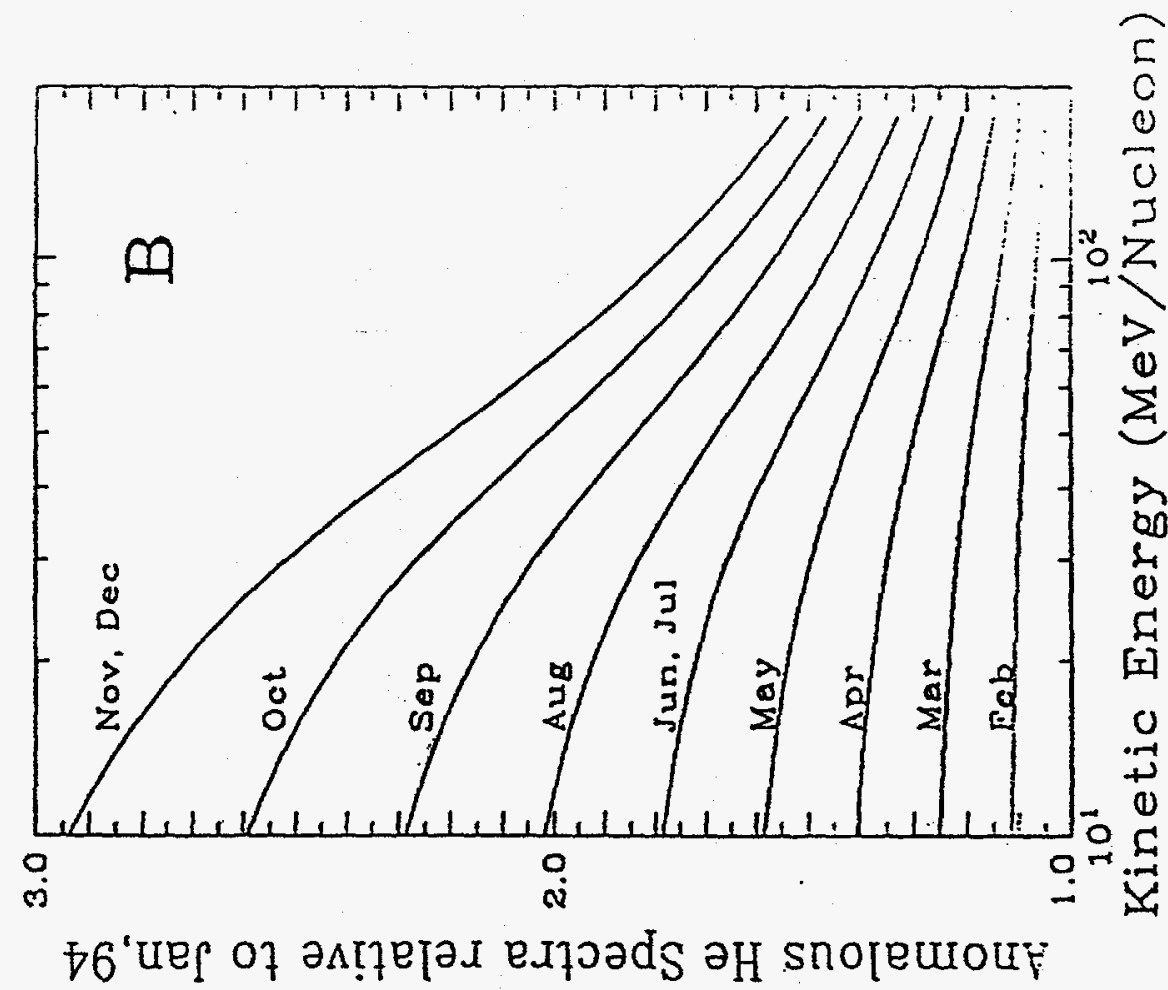

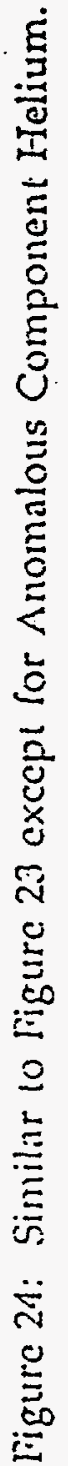



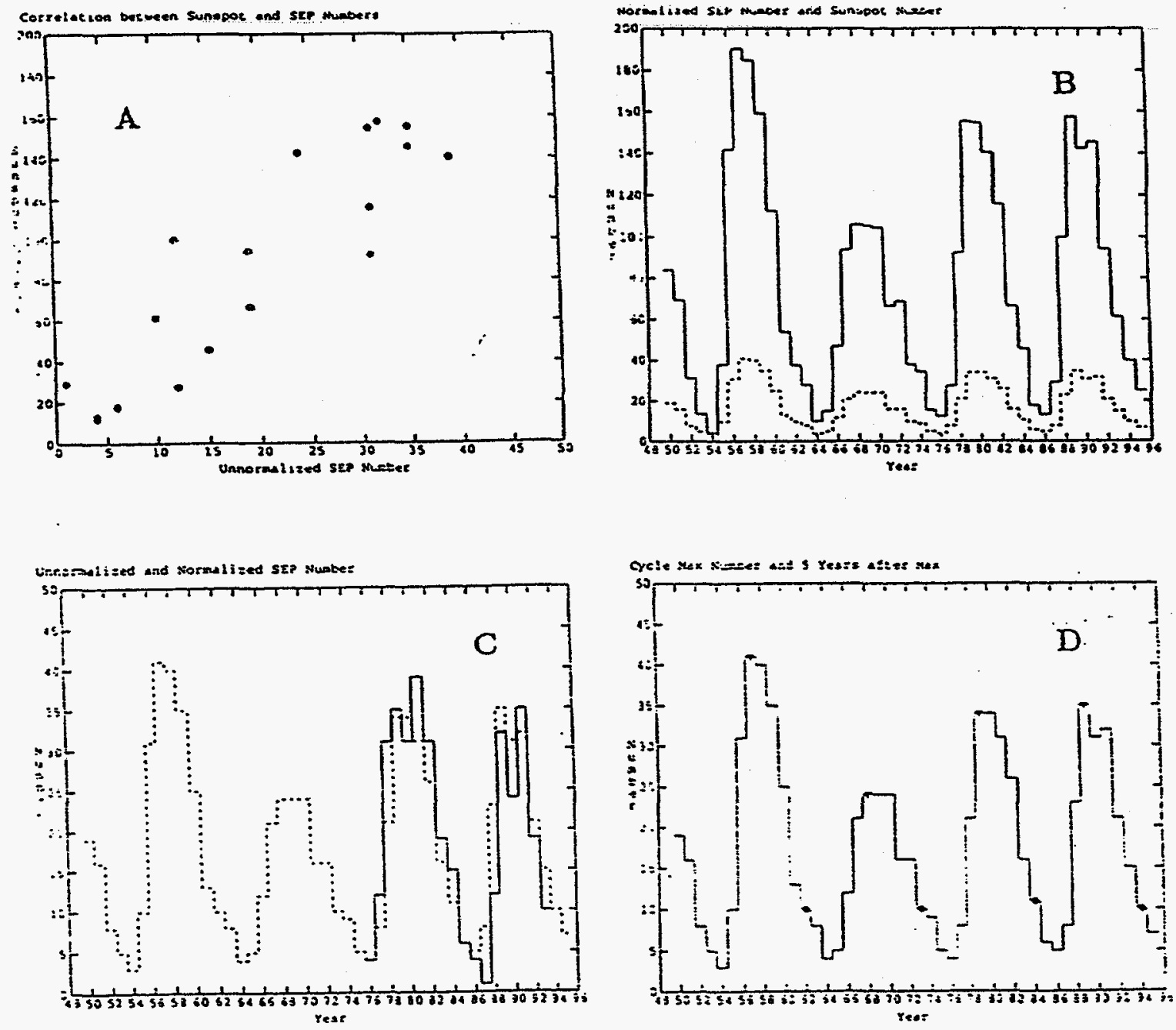

Figure 25: The time variation in the number of SEP events. Panel A (upper left) shows a correlation plot of the mean sunspot number versus number of SEP events observed during 1976 to 1993 . Panel B shows the number of SEP events per year (dashed curve) inferred from the mean sunspot number (solid curve). A comparison of the observed (solid) and inferred (dashed) number of SEP events is shown in panel C (lower left). Finally, the inferred SEP event number is drawn in panel $D$ (lower right) with the peak number for each cycle (filled inverted triangle) and the number 5 years following the peak (filled circles) marked. 
peak of solar activity. Thus, also indicated in the panel as filleci circles is the SEP number expected for 1994 as well as the level for each year that is five years after the peak number. The plot shows that there is remarkable agreement for each of the corresponding years in the previous cycles. In fact, the number events per year for 5 or 6 years around solar minimum appears to be relatively independent of the number of events during solar maximum or the total number of events during the cycle. Thus, with reasonable confidence we can estimate that roughly $10 \pm 5$ SEP events might be observed by the CLEMENTINE I spacecraft during 1994.

An advantage of this approach to estimating the SEP number is that it takes into account the inefficiency of any particular spacecraft in observing all the SEP events which actually occur. Since the SEP energetic particles are generally tied to the interplanetary (solar) magnetic field lines, the direct particles will travel along the field line linking the spacecraft to the sun. For events which are not "directly connected", particles would have to diffuse across field lines. Thus, a given spacecraft can efficiently "view" only a limited region of the solar surface. Since solar flares and Coronal Mass Ejections (CME), which may generate SEP events, can occur anywhere on the sun, a single spacecraft will have the opportunity to "see" only a fraction of the total number of events. Further, only a portion of these solar events may generate energetic particles. which "escape" into interplanetary space. Finally, spacecraft instrument thresholds and geometrical factors limit the size and type of SEP events which can be detected to the larger events. These are the events, however, which likely provide the majority of the SEP contribution to the total dose and SEU. rates relevant to radiation environment studies. As these sensitivities and inefficiencies are very roughly the same for most current technology spacecraft sensors, scaling from the observed IMP-8 and GOES "event rate" will automatically take these effects into account for the CLEMENTINE I mission.

In addition to the number of events during 1994 we also need an expectation of the distribution of event sizes. Here we can examine the total. yearly fluence (particles $/ \mathrm{cm}^{2}$-sr) for $>10 \mathrm{MeV}$ protons in SEP events between 1976 and 1993 based upon the GOES and IMP-8 observations. These data are plotted in Figure 26. where each curve is for a particular fluence range and is obtained by:

[(Number of events in range) $\times$ (Mean fluence of range) $]+$ (Total fluence from lower ranges)

The fluence ranges are: $200-10^{4}$ (dot-dash-dash); $10^{4}-10^{5}$ (dot-dot-dash); $10^{5}-10^{6}$ (long dash); $10^{6}-10^{7}$ (medium dash); $10^{7}-10^{8}$ (short dash); an $\dot{i}>10^{8}$ (solid). The number distribution of events within these ranges is shown in the different panels of Figure 27. From these figures we can see that lowe: iluence events (< $10^{5}-10^{6}$ ) may decrease in number following the sunspot number peaks (1979, 1989), and there seems to be an increased chance that higher fluence events 


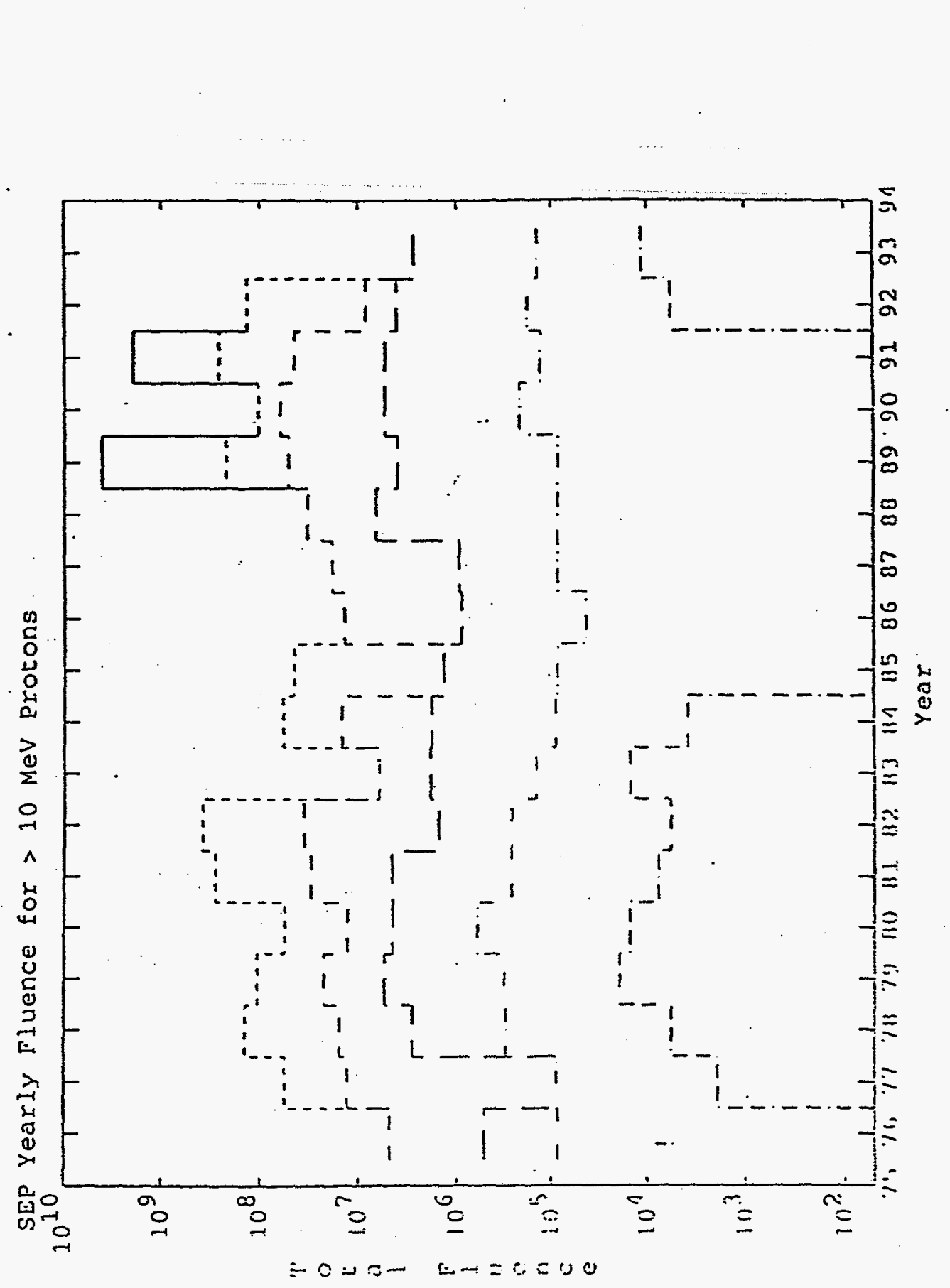

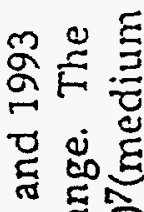

๙

의

동

\&

它安

造

os $\&$

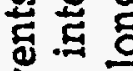

5

옥

똥

5

द व

농 농

농.? 인

$\rightarrow 0$ 。

象

$\sum_{0}$ 岂

$\wedge$ 约

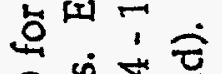

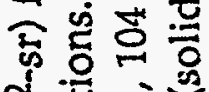

至

द्य

क षे

웡 은

象

요동

\&

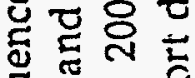

로 딴

웡

\& 500 ?

고을 촗

오웡

๑)

$\ddot{\circ}$

造 


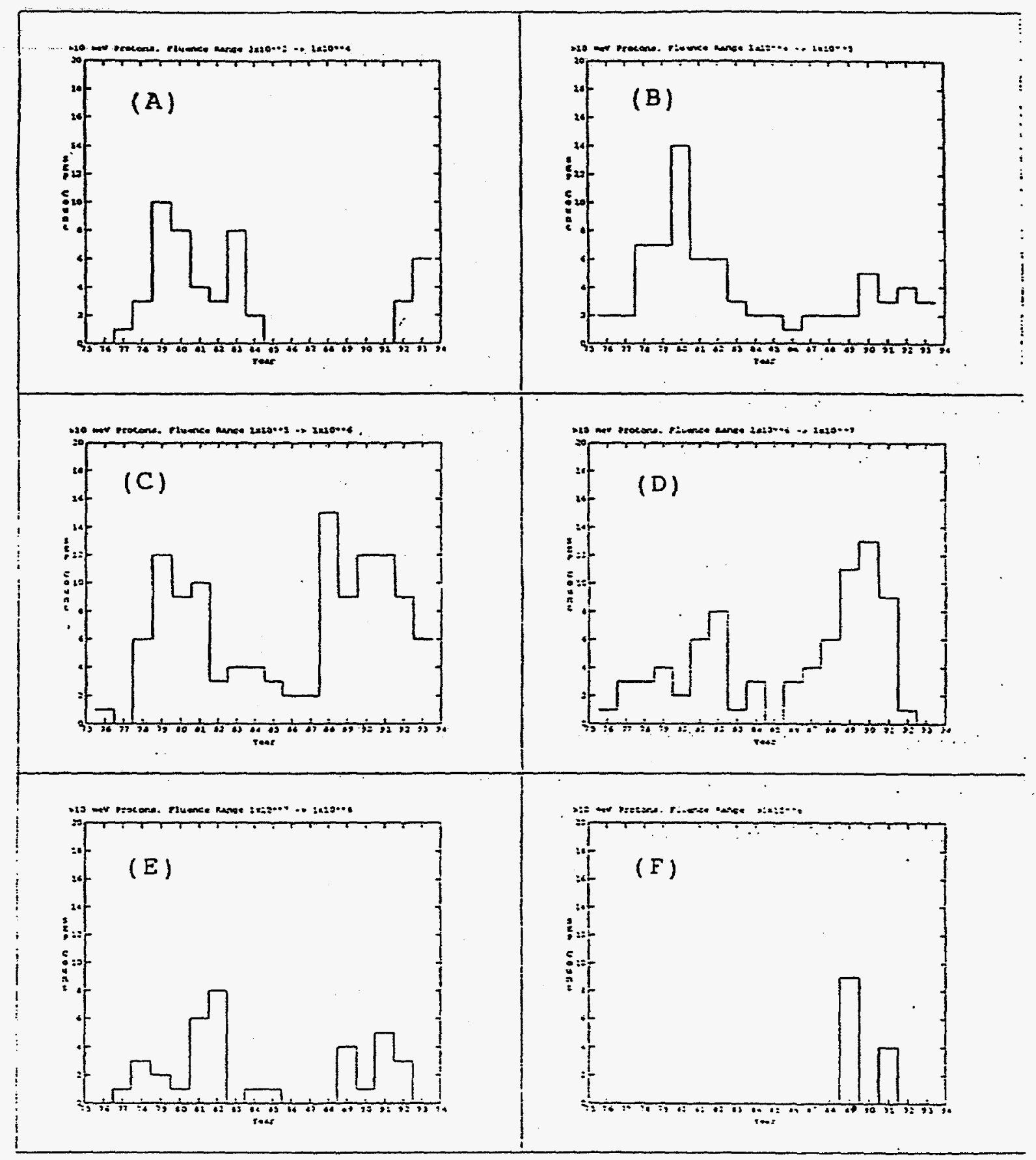

Figure 27: The number of SEP events within a particular fivence range as a function of time from 1976 to 1993. The fluence ranges are: $200-10^{4}$ (A), $10^{4}-10^{5}(\mathrm{~B}), 10^{5}-10^{6}(\mathrm{C}), 10^{6}-10^{7}(\mathrm{D}), 10^{7}-10^{5}(\mathrm{E})$, and $>10^{8}(\mathrm{~F})$. 
$\left(>10^{6}\right)$ occur during the declining phase of the cycle. Thus, the total proton fluence from SEP events can remain high, or even increase, for several years following the sunspot maximum.

To place a limit on the total fluence during the CLEMENTINE I mission we can, again, extrapolate from 1984 which is five years after the solar cycle peak of 1979. This extrapolation indicates that the total SEP fluence should be less than $10^{8}$ particles $/ \mathrm{cm}^{2} \mathrm{sr}$ for $>10 \mathrm{MeV}$ protons, but there is a chance for an event with $>10^{9}$ particles $/ \mathrm{cm}^{2}$-sr. We can also estimate the intensity distribution over the $\sim 10$ events expected for 1994 to be: $\sim 5$ events with a proton fluence of $<106, \sim 3$ with a fluence in the range $10^{6}$ to $10^{7}$, and $\sim 2$ where the proton fluence exceeds 107 .

\section{Expectations during 1994}

The number and proton intensity variations of the SEP events over time which can be examined from the historical record do not constrain other characteristics, such as the energy spectrum or heavy ion content, which are important in determining the LET. We have therefore constructed four sample collections of SEP events from the events contained in the CHIME model. Each of these samples contain 10 events and an intensity distribution consistent with that estimated for 1994. The heavy ion composition between the samples was varied to investigate the effect on the derived LET spectrum. The general . character of the sample as well as the events (listed in Tables 2 to 4 ) used to construct the sample are:

1. Heavy Ion Poor: In this sample all events have a heavy ion enrichment factor $(\delta)$ of less than about 0.1. The CHIME model SEP event numbers used for this sample are: $1,3,8,9,13,18,22,23,24$, and 25 .

2. Heavy Ion Rich: Two events in this sample have a heary ion enrichment factor $(\delta)$ of greater than about 0.2 . The CHIME model SEP event numbers used for this sample are: $4,5,8,9,13,14,15,16,23$, and 26.

3. Typical: The composition distribution of this sample is similar to that of the 26 events of the CHIME model. One event has a heavy ion enrichment factor $(\delta)$ of greater than about 0.1 . The CHIME model SEP event numbers used for this sample are: $2,3,6,8,14,15,17,22,23$, and 26 .

4. Large: Here the composition distribution is the same as in the "Typical" sample, with the exception that it includes the largest erent seen by the CRRES/SPACERAD mission. The CHIME model SEP event numbers used for this sample are: $1,3,8,9,10,13,18,22,24$, and 25. 
The characteristics of the four samples are illustrated in Figure 28. This figure shows, in the left hand panels, the proton (solid) and Helium (dashed) fluence spectra and, in the right hand panels, the relative elemental composition. The composition differences between the sample can be more clearly seen in Figure 29 where the abundance for each element relative to the "Typical" sample (i.e. \#3) is plotted versus element charge. The solid curve is for the Heavy Ion Poor sample (\#1) and the highest charge elements $(24<\mathrm{Z}<$ 28) are repressed relative to "typical" composition by about a factor of five. The short dash curve is for the Heavy Ion Rich sample (\#2) which shows that lighter elements $(2<Z<8)$ are repressed about $60 \%$ while heavy elements are enhanced about the same amount. Finally, the long dash curve is for the Large (\#4) sample. Here the enhancement varies from about a factor of 7 for protons and about a factor of 3 for all other species.

\section{The Geomagnetic Transmission of Interplanetary Particles}

The geomagnetic field shields the Earth and objects in low Earth orbit from the full effects of the interplanetary radiation environment. The highly eccentric orbit of the Interstage Adapter carries the ISA deep. within the magnetosphere, but most of the orbit period is spent at high magnetic $L$ values where interplanetary energetic particles have relatively easy access. Thus, the calculated geomagnetic transmission function, averaged over the ISA orbit and shown in Figure 30, indicates that interplanetary energetic particles with rigidity greater than $100 \mathrm{MV}$ should have a $90 \%$ or greater transmission through the Earth's geomagnetic field.

The rigidity of a species is proportional to the mass to charge ratio (A/Z) of the particle and more "rigid" species are less affected by the Earth's magnetic field. This effect is shown in Figure 31, where the ratio of the energy spectra seen in interplanetary space relative to that averaged over the ISA orbit is plotted. The solid curve is for protons with $A / Z=1$, the short dashed curve is for Helium and other heavy ions with $A / Z=2$, and the long dashed curve is for heavy ions with $2.18<\mathrm{A} / Z<2.4$. The largest effect is for protons at $10 \mathrm{MeV}$ where the interplanetary flux is about $7 \%$ higher than the averaged flux at the ISA. As heavier ions are more rigid they have a much higher average transmission and, at most, there is a $2 \%$ decrease in the flux for the lowest energies considered here. As the Anomalous Component species are expected to be only singly ionized they have very high $A / Z$ ratios and, thus, would be affected by the geomagnetic field only at energies below $10 \mathrm{MeV} /$ nucleon. Finally, at high energy (i.e. high rigidity) geomagnetic transmission is still. somewhat less than $100 \%$ due to the "shadow" caused by particles intercepting the Earth (see section III.B.4) 


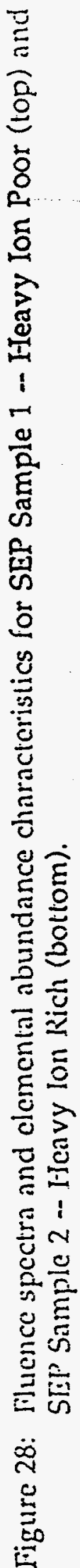




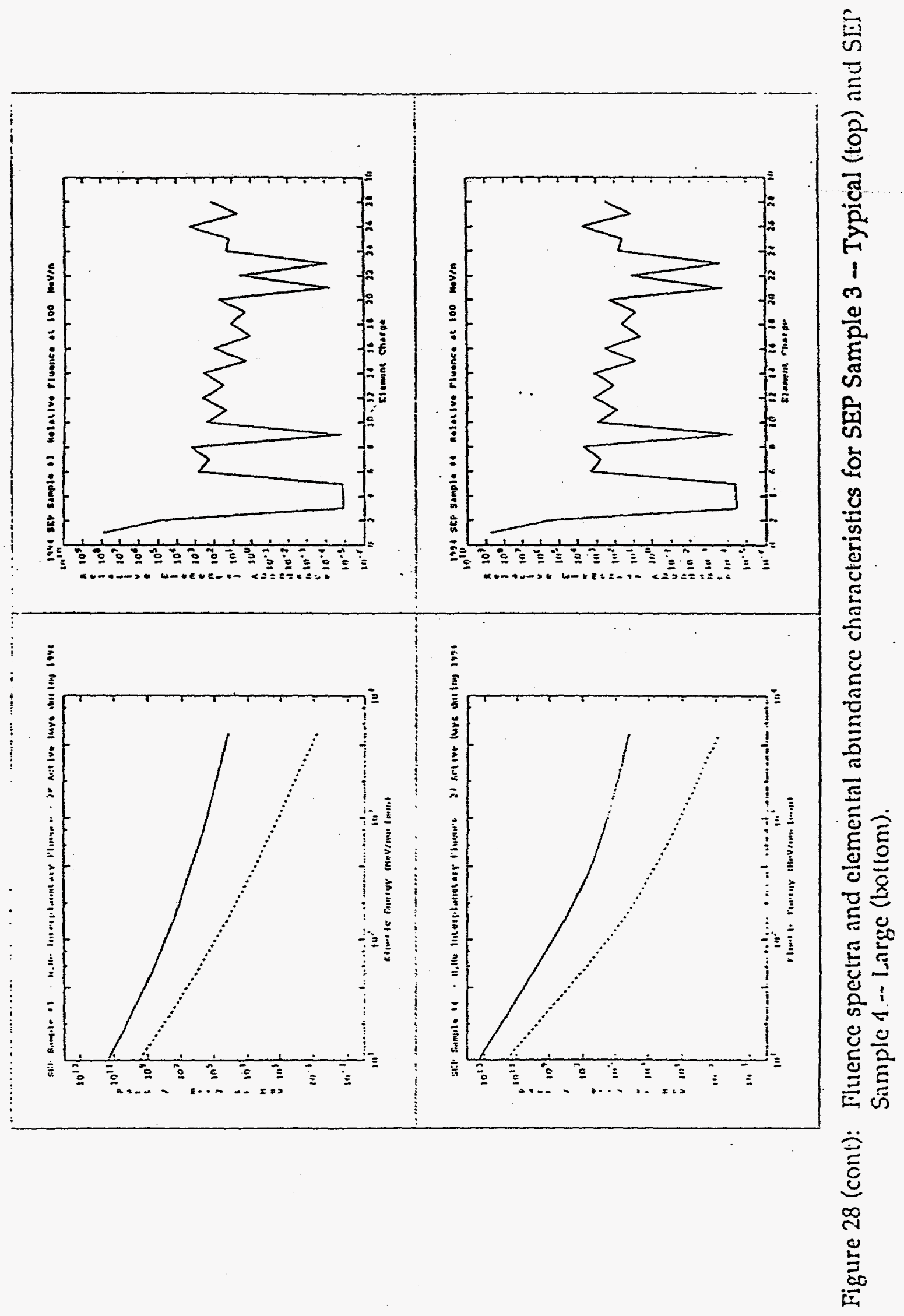




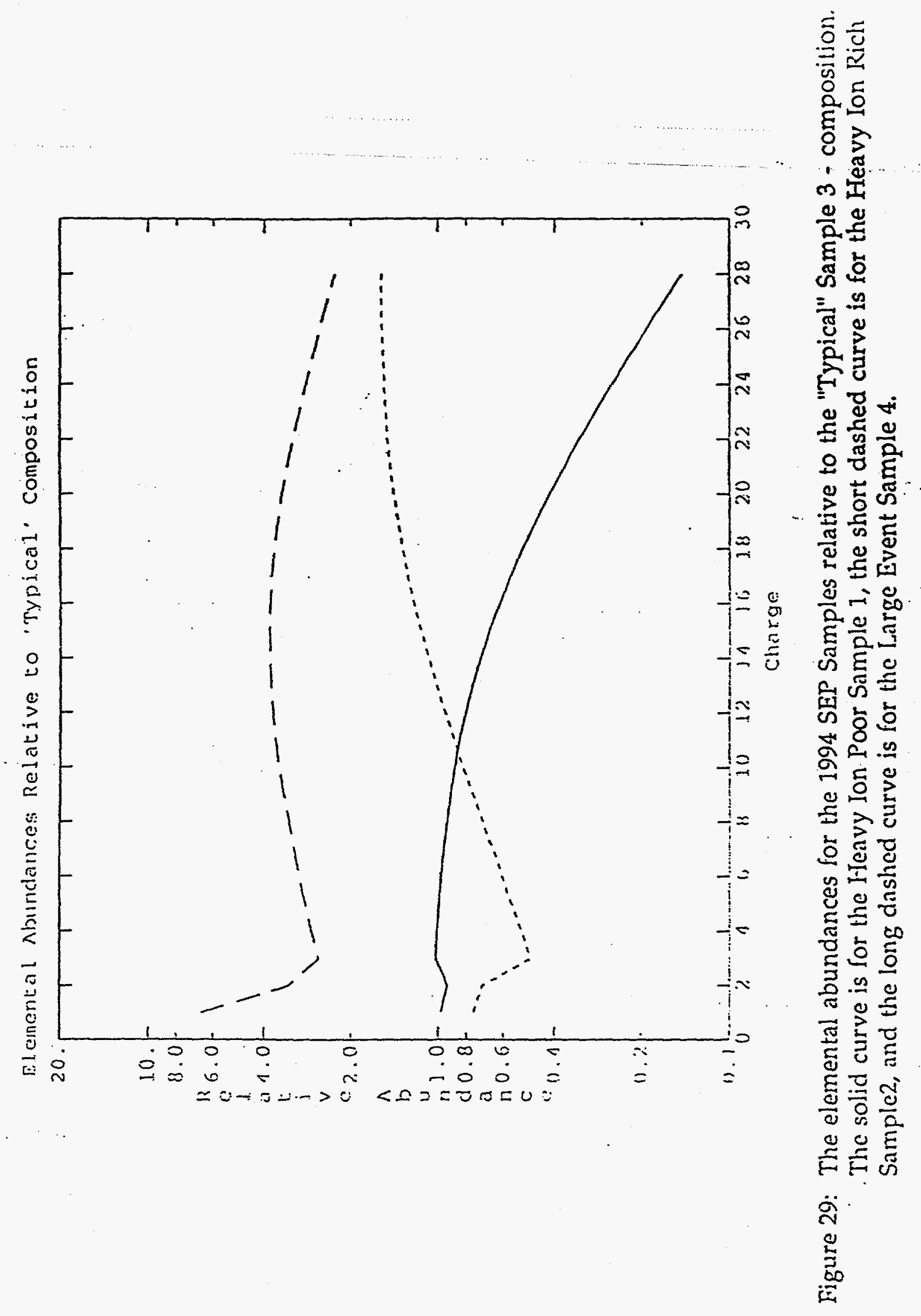




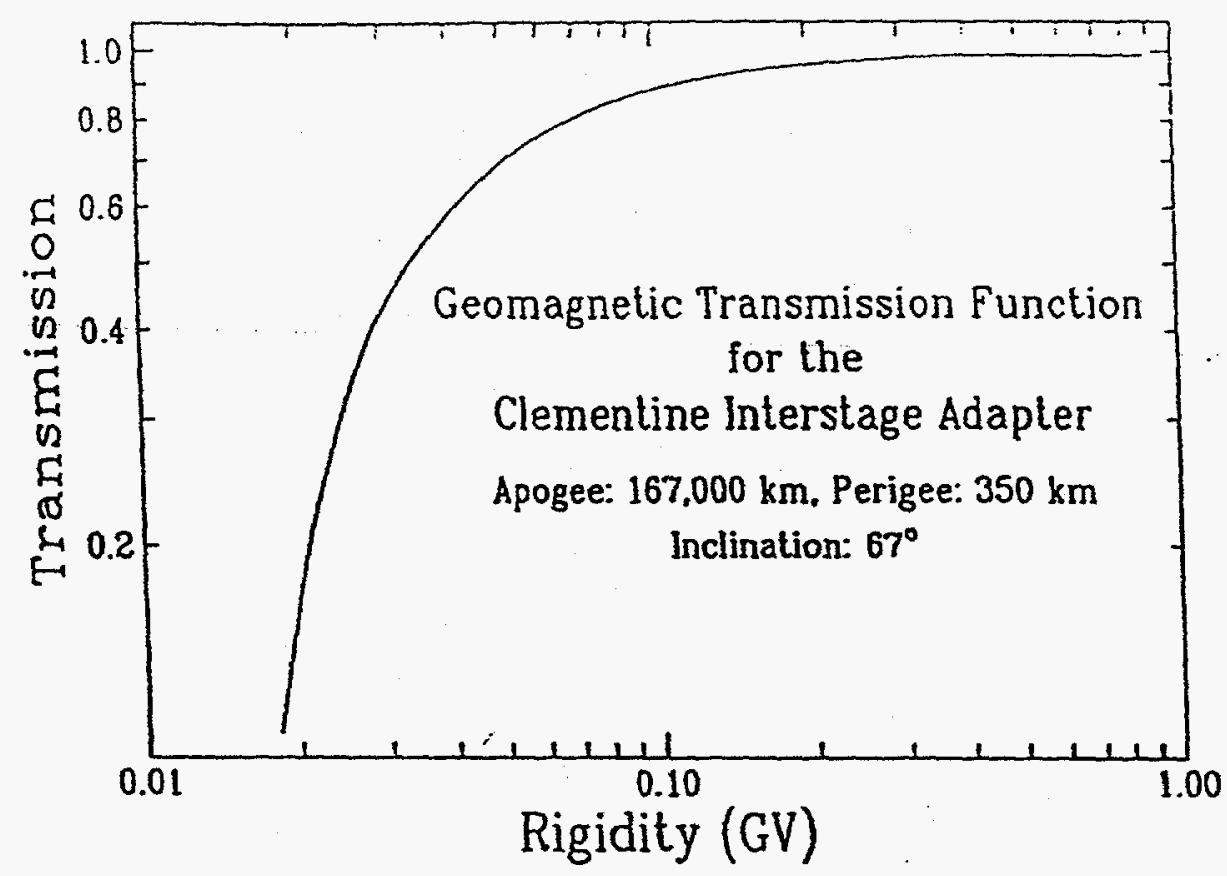

Figure 30. The geomagnetic transmission function calculated for the Clementine Interstage Adapter orbit.

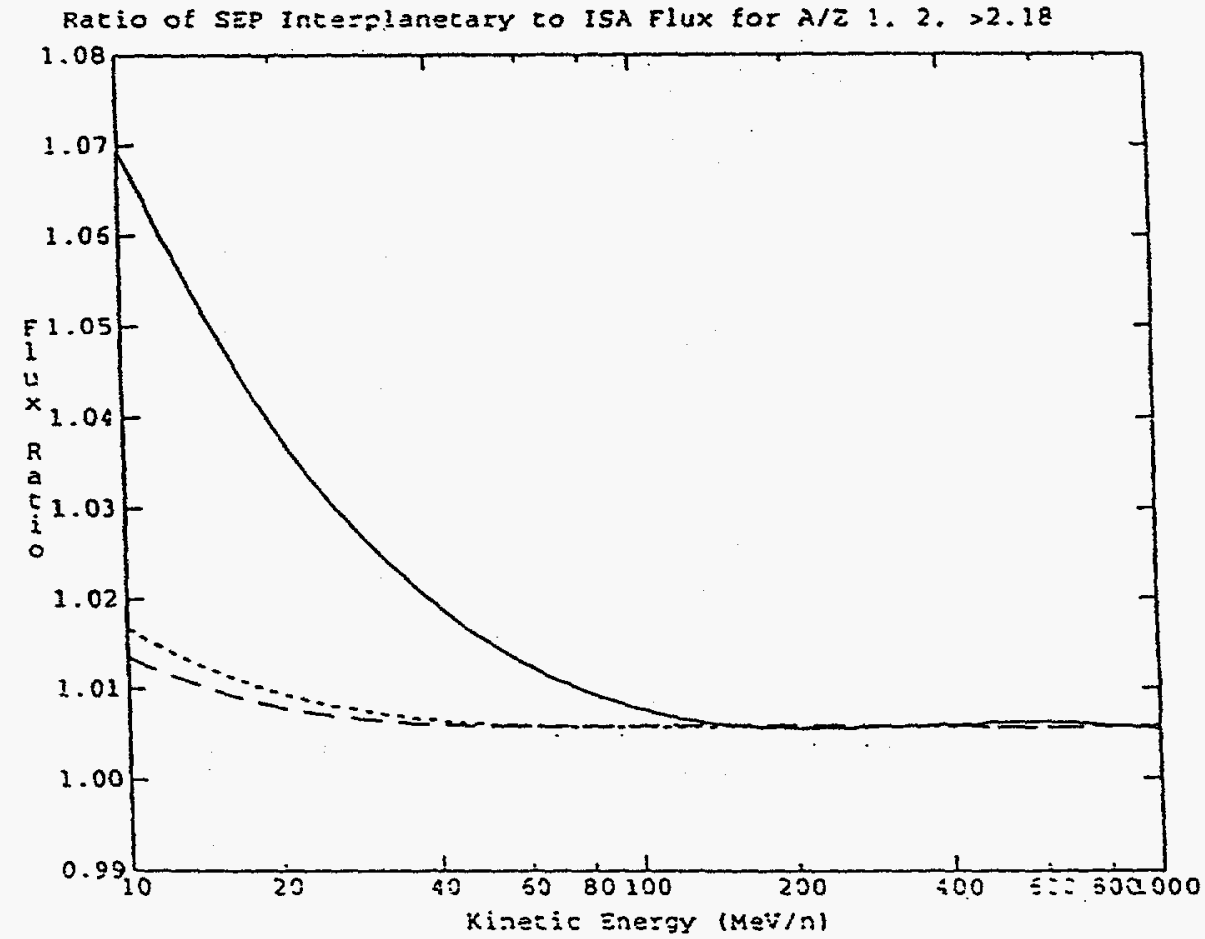

Figure 31. Ratio of particle energy spectra seen in interplanetary space relative to that averaged over the Interstage Adapter orbit. The solid curve is for protons with $A / Z=1$, the short dashed curve is for Helium and other heavy ions with $A / Z=2$, and the long dashed curve is for heavy ions with $2.18<\mathrm{A} / Z<2.40$. 


\section{E. The Linear Energy Transfer (LET) Functions}

With the radiation environment now estimated for all three phases of the CLEMENTINE I mission, we can now derive the Linear Energy Transfer (LET) spectrum and investigate its implications. The LET was calculated using the CHIME model as described in section III.B.5 and is presented here as plots of integrated flux (particles $/ \mathrm{m}^{2} \mathrm{~s}-\mathrm{sr}$ ) or fluence (particles $/ \mathrm{m}^{2}-\mathrm{sr}$ ) versus the LET $\left(\mathrm{MeV} / \mathrm{mg} / \mathrm{cm}^{2}\right)$.

\section{Effects of Detector and Shield Thickness}

The derived LET spectra can be strongly influenced by the shielding material surrounding the "target" sensitive layer and the thickness of the sensitive layer, since different species in the radiation environment lose energy at different rates. The sensitivity of the LET for a particular SEP event (\#5 from Tables $2-4$ ) to the shield thickness is shown in Figure 32. In this figure the LET was calculated for a 1 micron thick Silicon sensitive layer and for a series of $\mathrm{Al}$ shields that range in thickness from 1 mil (top most curve) to 1600 mils (bottom curve). The top most curve represents the LET for, essentially, an unshielded detector and the integrated flux is quite large. However, introducing only a thin, 50 mil $\left(\sim 0.34 \mathrm{~g} / \mathrm{cm}^{2}\right)$ shield reduces this flux by 3 to 5 orders of magnitude. Thus, while SEP events can produce copious amounts of energetic particles for short periods of time, their relatively steep energy spectrum means that these particles are not very penetrating and can be effectively shielded by moderately thin amounts of material.

Galactic cosmic rays are much more penetrating than SEP's, since the GCR flux peaks at $300-600 \mathrm{MeV} /$ nucleon and remains relatively high up to energies $>1000 \mathrm{MeV} /$ nucleon (c.f. Figures 2 and 14). Thus, we would expect that the GCR LET would be much less sensitive to the shield thickness. This is illustrated in Figure 33 for GCR spectra modulated to $\phi=558 \mathrm{MV}$ and used to calculate the LET spectrum in a 1 micron Silicon sensitive layer. The top curve in the figure, once again, corresponds to essentially no shielding and is the LET spectrum for a $1 \mathrm{mil}\left(\sim 7 \mathrm{mg} / \mathrm{cm}^{2}\right)$ Al shield. The large peak in the spectrum between 1 and 10 $\mathrm{MeV} /\left(\mathrm{mg} / \mathrm{cm}^{2}\right)$ is due to the anomalous component (see below). The next curve down is the LET spectrum for a shield thickness of about $12.5 \mathrm{~g} / \mathrm{cm}^{2}(\sim 1850$ mils of Al) and, other than the absence of the AC peak, there is little difference from the unshielded case. Due to this insensitivity we have also included in Figure 33 example LET spectra calculations for significantly thicker shields ranging up to $\sim 400 \mathrm{~g} / \mathrm{cm}^{2}$. At these thicknesses GCR interactions in the shield are an important factor and, as previously mentioned, CHIME does not include these in the calculations. Nevertheless, Figure 33 indicates that it is extremely difficult to reduce the GCR induced LET by merely inceasing the shield thickness. 


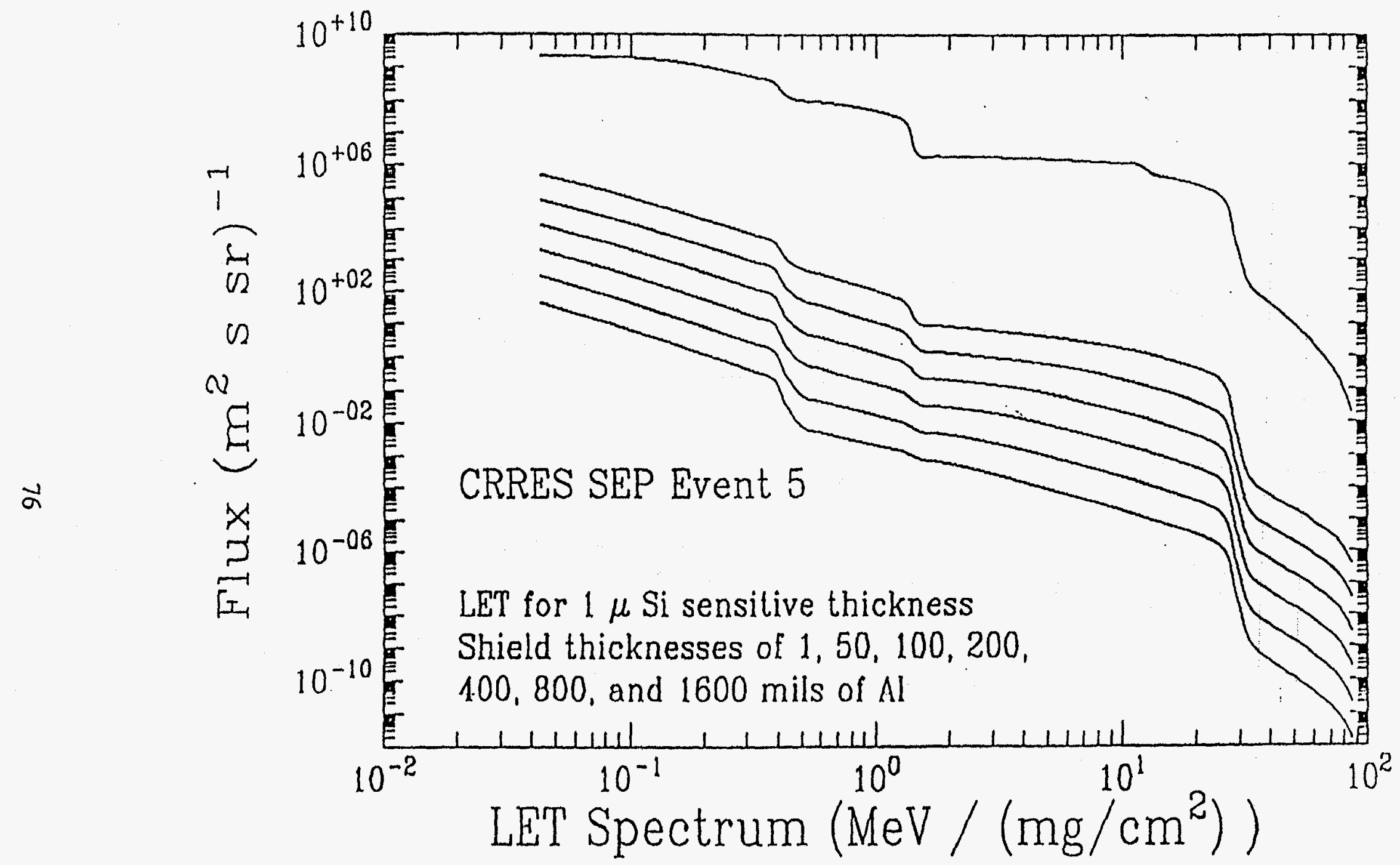

Figure 32: The integral Linear Energy Transfer (LET) spectrum of a SEP event for a 1 micron thick Silicon sensitive layer as a function of shield thickness. The curves from the top down are for Aluminum shields that are $1,50,100,200,400,800$ and 1600 mils thick. Note that only a modest amount of shielding will significantly reduce the SEP LET flux. 


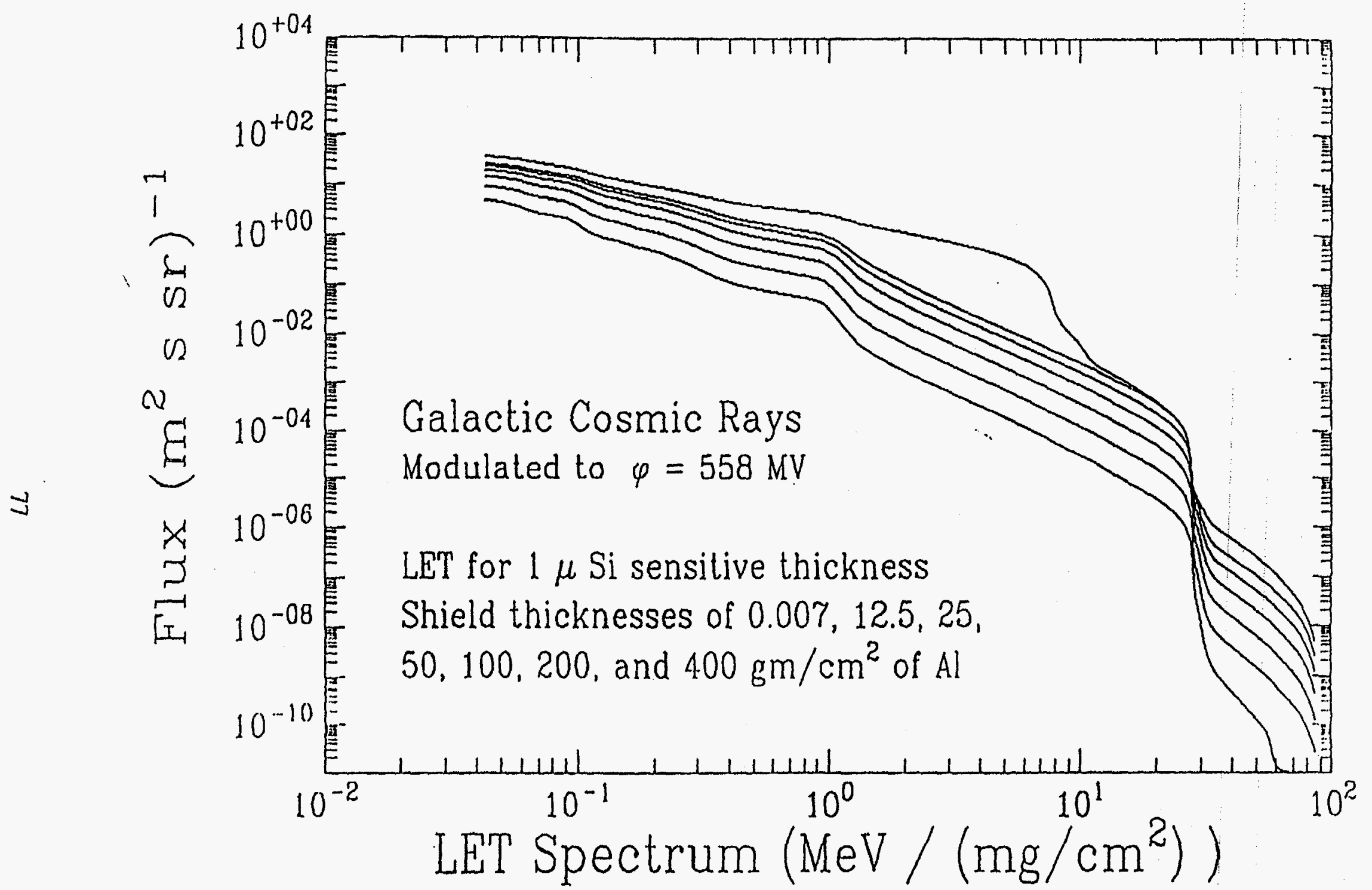

Figure 33: The integral Linear Energy Transfer (LET) spectrum of Galactic Cosmic Rays modulated to $\phi \leq 558 \mathrm{MV}$ for a 1 micron thick Silicon sensitive layer as a function of shield thickness (see text for details). 


\section{Interplanetary phases (Lunar and Geographos)}

The LET calculated for the CLEMENTINE I phases depends upon whether or not the AC is included, the type of SEP events expected during the mission and the level of solar modulation. Figure 34 shows the effect of including the $A C$ in the LET calculation. Both panels of the figure use the same GCR interplanetary spectra with a 1 micron Silicon sensitive layer and Al shield thicknesses (from top to bottom) of 1, 50, 100, 200, 400, 800 and 1600 mils. The top panel (A) does not include the anomalous spectra while the bottom panel (B) does. Only in the thin shield ( 1 mil) case is the AC evident, in panel B, as the significant increase in flux between LETs of 1 to $10 \mathrm{MeV} /\left(\mathrm{mg} / \mathrm{cm}^{2}\right)$. Increasing the shield thickness to 50 mils $\left(\sim 0.3 \mathrm{~g} / \mathrm{cm}^{2}\right)$ effectively removes these particles. This is due to the AC's very steep energy spectrum (see sections II.B and III.B.1).

In Figure 35 the LET fluence (particles / $\left(\mathrm{m}^{2} \mathrm{sr}\right)$ ) spectrum for the four SEP samples described above are shown. These LET spectra are for a 1 micron Si sensitive layer thickness and, again, from top to bottom, 50, 100, 200, 400, 800 and 1600 mil Al shields. Each panel is for a different SEP sample: (A) Typical; (B) Heavy Ion Rich; (C) Heavy Ion Poor; and (D) Large. In general there are only slight differences between the LET spectra in panels A, B and C. These differences are mostly at LET values greater that $3-4 \mathrm{MeV} /\left(\mathrm{mg} / \mathrm{cm}^{2}\right)$ reflecting the relative difference in heavy ion content between the samples. For example, at $\sim 10 \mathrm{MeV} /\left(\mathrm{mg} / \mathrm{cm}^{2}\right)$ the Heavy Ion Rich sample (B) fluence is about an order of magnitude larger than that for the Heavy Ion Poor sample. The spectra in panel $D$, however, show a considerably enhanced fluence for all LET. This is due to the single, big event included in the sample.

The LET fluence spectra for the Lunar Survey period $(2 / 21 / 94-5 / 3 / 94)$ and the Geographos encounter period $(5 / 27 / 94-8 / 31 / 94)$ are shown in, respectively, in Figures 36 and 37. These spectra were calculated for the same sensitive layers and shield thickness used for Figure 35 and include all components of the interplanetary radiation environment, the $A C$ as well as the "Typical" SEP sample, appropriately scaled for the time period, and GCR with modulation levels of $588 \mathrm{MV}$ for the lunar orbit period and $558 \mathrm{MV}$ for the asteroid flyby. These LET spectra are dominated by the GCR, but the SEP effects are strongly evident for LET values less than $1 \mathrm{MeV} /\left(\mathrm{mg} / \mathrm{cm}^{2}\right)$ and for shields less than 300 mils thick. For reference these composite LET spectra are provided here as Table 6 for the Lunar Survey period and Table 7 for the Geographos encounter phase. 

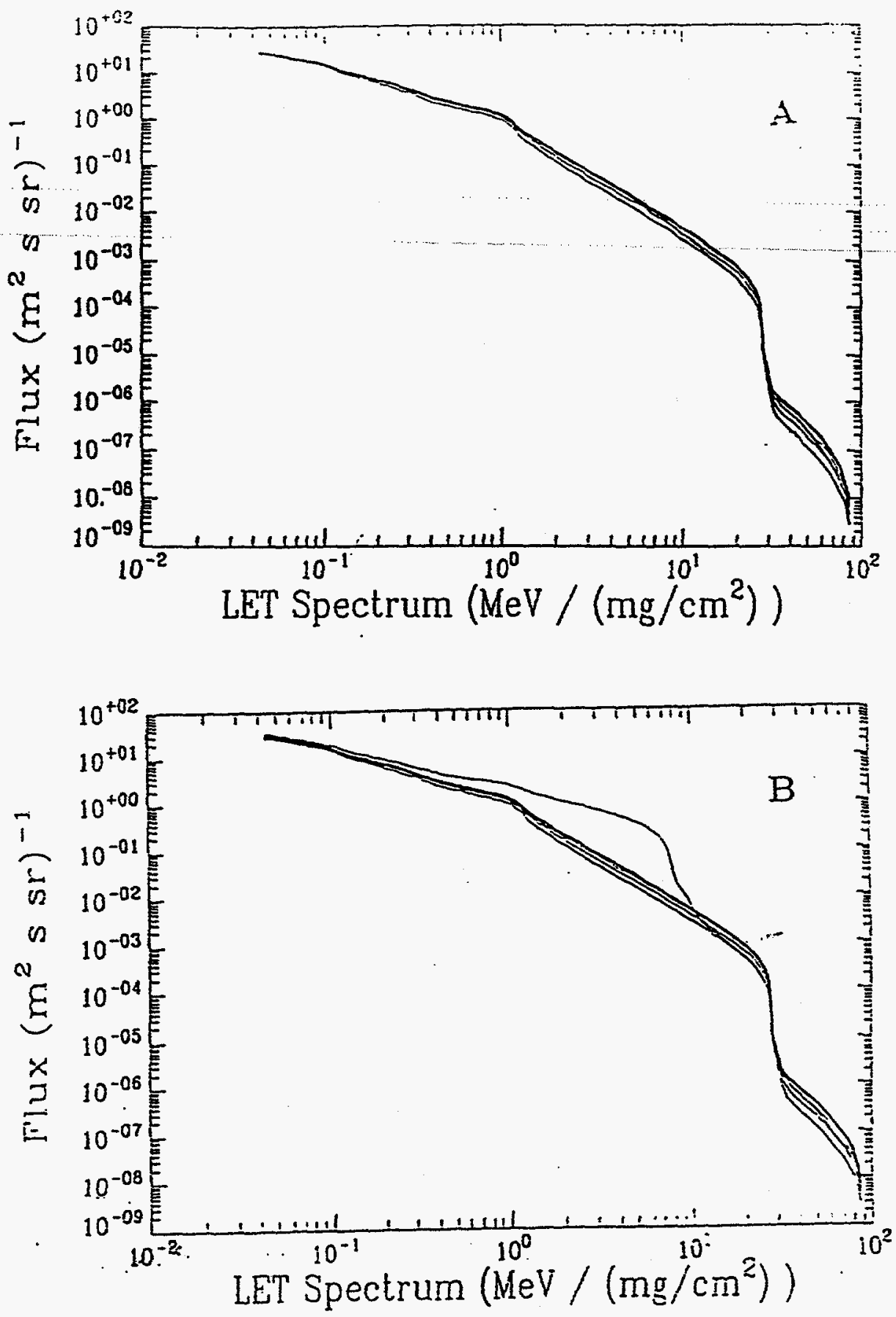

Figure 34: The Galactic Cosmic Ray LET spectrum for a 1 micron Silicon sensitive layer and Aluminum shield thicknesses of $i, 50,100,200$, 400,800 and 1600 mils. The bottom panel (B) inclucies Anomalous $\mathrm{He}, \mathrm{N}, \mathrm{O}$ and $\mathrm{Ne}$ while the top panel (A) does not. The effects of the Anomalous species is evident when the shield thickness is only 1 mil thick, and is effectively blocked by a shield only 50 mils thickness. 


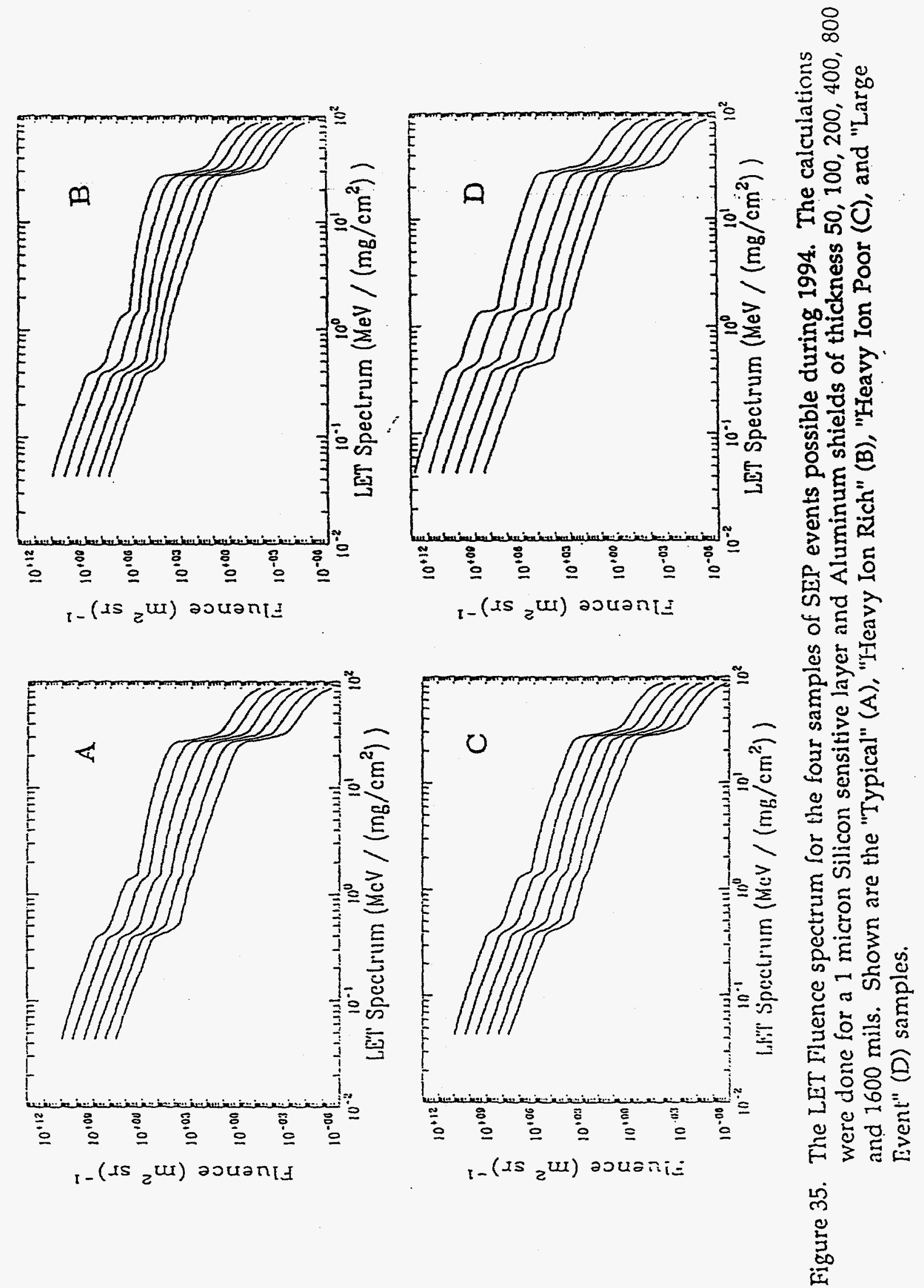




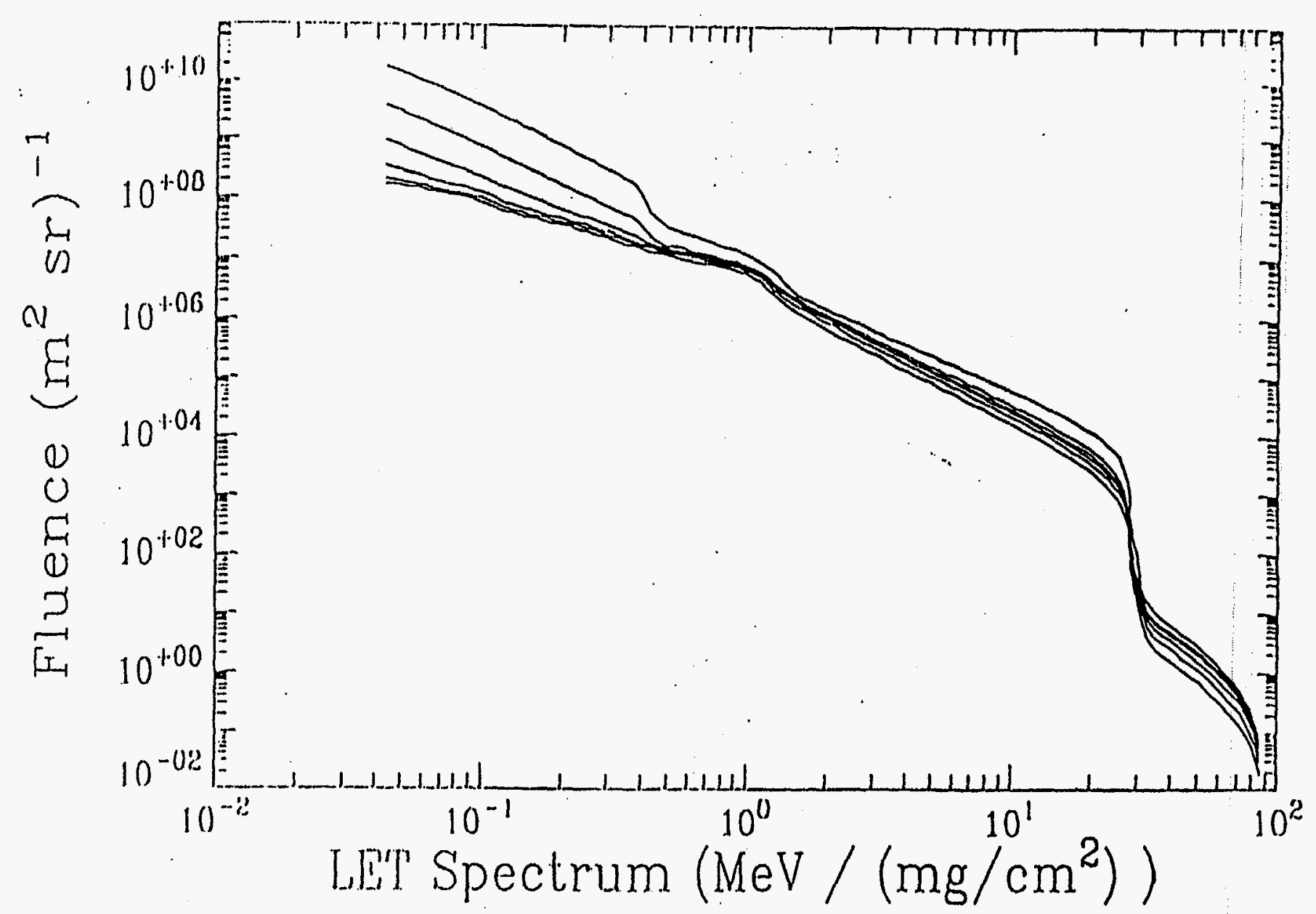

Figure 36: The LET fluence spectrum calculated for the Lunar survey period of the Clementine mission (2/21/94 $5 / 3 / 94$ ) is shown for a 1 micron Silicon sensitive layer and shields of 50, 100,200,400,800 and 1600 mils of Aluminum. The particle flux used in this calculation includes Galactic Cosmic Rays and Anomalous species with a solar modulation level of $\phi=588 \mathrm{MV}$ as well as the "Typical" SEP sample fluence appropriately scaled for time period. 


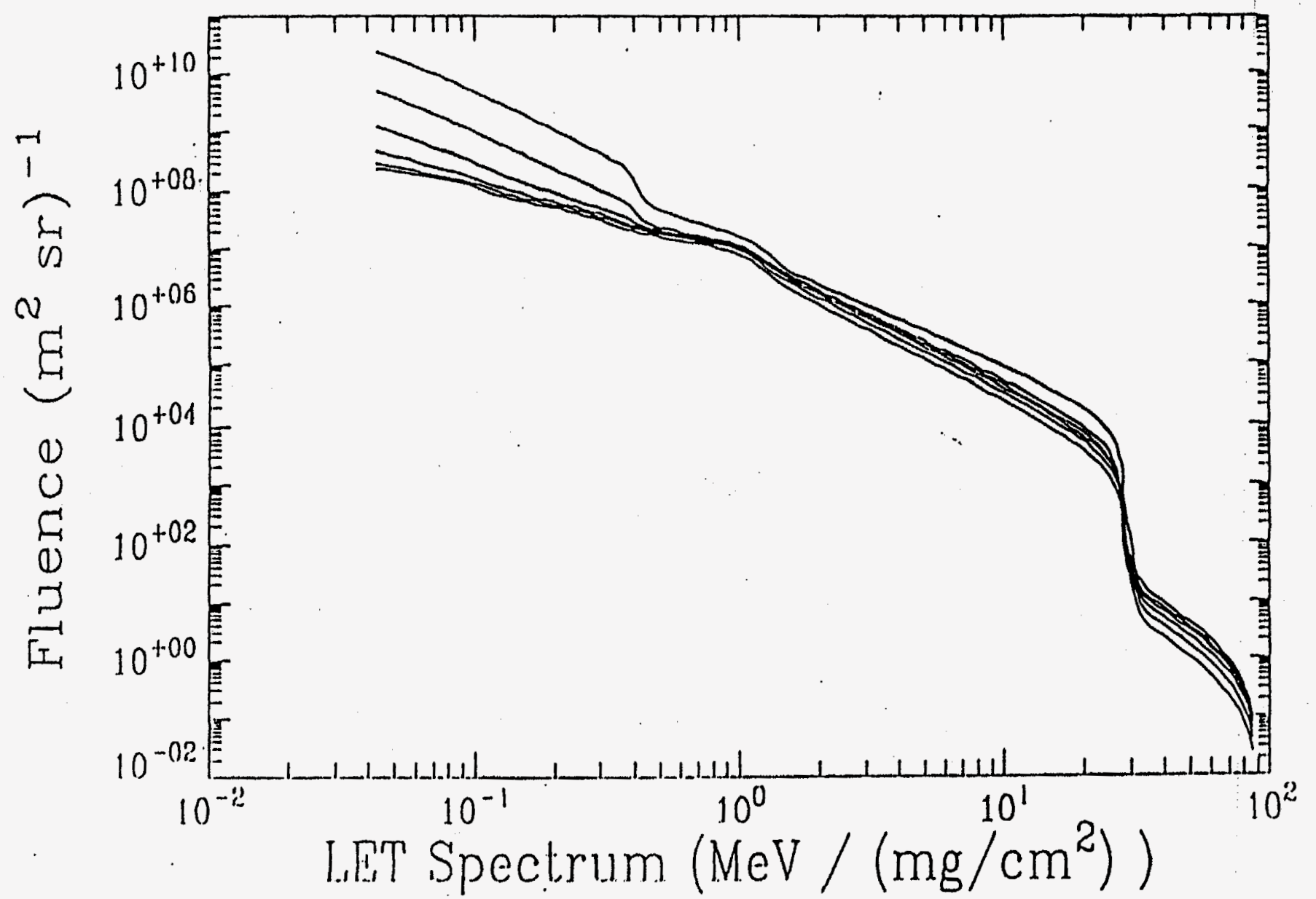

Figure 37: Similar to Figure 36 except calculated for the Clementine spacecraft Geographos encounter time period $(5 / 27 / 94-8 / 31 / 94)$. The lower level of solar modulation $(\phi=588 \mathrm{MV})$ causes a slight increase in fluence for a given LET as compared to the Lunar survey time period. 
Table 6: LET Fluence Spectra for Lunar Survey Period

$\mathrm{MeV} /$ ( $\mathrm{mg} / \mathrm{cm}^{2}$ )

$\begin{array}{lll}0.043 & 1.84 E+10 & 3.91 E+09 \\ 0.050 & 1.42 E+10 & 2.94 E+09 \\ 0.059 & 1.08 E+10 & 2.18 E+09 \\ 0.068 & 8.03 E+09 & 1.60 E+09 \\ 0.080 & 5.89 E+09 & 1.18 E+09 \\ 0.093 & 4.29 E+09 & 8.62 E+08 \\ 0.109 & 3.08 E+09 & 6.20 E+08 \\ 0.127 & 2.19 E+09 & 4.42 E+08 \\ 0.148 & 1.57 E+09 & 3.21 E+08 \\ 0.173 & 1.08 E+09 & 2.27 E+08 \\ 0.202 & 7.56 E+08 & 1.65 E+08 \\ 0.236 & 5.27 E+08 & 1.20 E+08 \\ 0.276 & 3.67 E+08 & 8.71 E+07 \\ 0.322 & 2.56 E+08 & 6.39 E+07 \\ 0.377 & 1.73 E+08 & 4.56 E+07 \\ 0.440 & 5.18 E+07 & 2.22 E+07 \\ 0.513 & 3.27 E+07 & 1.72 E+07 \\ 0.600 & 2.61 E+07 & 1.47 E+07 \\ 0.700 & 2.06 E+07 & 1.25 E+07 \\ 0.818 & 1.66 E+07 & 1.08 E+07 \\ 0.955 & 1.32 E+07 & 9.12 E+06 \\ 1.115 & 9.45 E+06 & 6.65 E+06 \\ 1.302 & 5.65 E+06 & 3.95 E+06 \\ 1.521 & 2.93 E+06 & 2.51 E+06 \\ 1.776 & 2.07 E+06 & 1.72 E+06 \\ 2.074 & 1.48 E+06 & 1.20 E+06 \\ 2.422 & 1.07 E+06 & 8.41 E+05 \\ 2.829 & 7.81 E+05 & 5.93 E+05 \\ 3.303 & 5.74 E+05 & 4.20 E+05 \\ 3.858 & 4.24 E+05 & 2.99 E+05 \\ 4.505 & 3.15 E+05 & 2.13 E+05 \\ 5.261 & 2.34 E+05 & 1.52 E+05 \\ 6.144 & 1.73 E+05 & 1.08 E+05 \\ 7.174 & 1.26 E+05 & 7.63 E+04 \\ 8.378 & 8.95 E+04 & 5.32 E+04 \\ 9.784 & 6.58 E+04 & 3.75 E+04 \\ 11.426 & 4.76 E+04 & 2.61 E+04 \\ 13.343 & 3.31 E+04 & 1.75 E+04 \\ 15.582 & 2.29 E+04 & 1.18 E+04 \\ 18.197 & 1.57 E+04 & 7.83 E+03 \\ 21.250 & 9.98 E+03 & 4.79 E+03 \\ 24.816 & 5.09 E+03 & 2.29 E+03 \\ 28.980 & 1.38 E+02 & 6.05 E+01 \\ 33.843 & 1.14 E+01 & 8.64 E+00 \\ 39.522 & 6.34 E+00 & 5.04 E+00 \\ 46.153 & 3.66 E+00 & 2.99 E+00 \\ 53.898 & 2.13 E+00 & 1.75 E+00 \\ 05.837 & 1.06 E+00 & 8.87 E-01 \\ 0.532 & 4.63 E-01 & 3.89 E-01 \\ 0.39 E-02 & 6.25 E-02\end{array}$

$200 \mathrm{mil}$

Al Shield

$9.44 \mathrm{E}+08$

$7.24 \mathrm{E}+08$

$5.54 \mathrm{E}+08$

$4.19 \mathrm{E}+08$

$3.23 \mathrm{E}+08$

$2.51 \mathrm{E}+08$

$1.87 \mathrm{E}+08$

$1.39 \mathrm{E}+08$

$1.08 \mathrm{E}+08$

$8.26 \mathrm{E}+07$

$6.58 \mathrm{E}+07$

$5.25 \mathrm{E}+07$

$4.10 \mathrm{E}+07$

$3.24 \mathrm{E}+07$

$2.49 \mathrm{E}+07$

$1.78 \mathrm{E}+07$

$1.50 \mathrm{E}+07$

1.30E+07

$1.12 \mathrm{E}+07$

$9.82 E+06$

$8.42 \mathrm{E}+06$

$6.13 \mathrm{E}+06$

$3.61 \mathrm{E}+06$

$2.36 \mathrm{E}+06$

$1.60 \mathrm{E}+06$

1.11E+06

$7.72 \mathrm{E}+05$

$5.40 \mathrm{E}+05$

$3.79 \mathrm{E}+05$

$2.67 \mathrm{E}+05$

$1.88 \mathrm{E}+05$

$1.33 \mathrm{E}+05$

$9.36 \mathrm{E}+04$

$6.56 \mathrm{E}+04$

$4.55 \mathrm{E}+04$

$3.18 \mathrm{E}+04$

$2.20 \mathrm{E}+04$

$1.47 \mathrm{E}+04$

$9.77 \mathrm{E}+03$

$6.44 \mathrm{E}+03$

$3.89 E+03$

$1.81 \mathrm{E}+03$

$4.66 \mathrm{E}+01$

$7.46 \mathrm{E}+00$

$4.37 \mathrm{E}+00$

$2.59 \mathrm{E}+00$

$1.51 \mathrm{E}+00$

7.66E-01

3.36E-01

5.37E-02
$400 \mathrm{mil}$

Al Shield

$3.40 \mathrm{E}+08$

$2.83 E+08$

$2.36 \mathrm{E}+08$

$1.89 \mathrm{E}+08$

$1.60 \mathrm{E}+08$

$1.35 \mathrm{E}+08$

$1.05 \mathrm{E}+08$

$8.15 \mathrm{E}+07$

$6.74 \mathrm{E}+07$

$5.54 \mathrm{E}+07$

$4.69 \mathrm{E}+07$

$3.94 \mathrm{E}+07$

$3.20 \mathrm{E}+07$

$2.60 \mathrm{E}+07$

$2.05 \mathrm{E}+07$

$1.65 \mathrm{E}+07$

$1.41 \mathrm{E}+07$

$1.23 \mathrm{E}+07$

$1.06 \mathrm{E}+07$

$9.30 \mathrm{E}+06$

$.7 .98 \mathrm{E}+06$

$5.77 \mathrm{E}+06$

$3.34 \mathrm{E}+06$

$2.18 \mathrm{E} \div 06$

$1.47 \mathrm{E}+06$

$1.01 \mathrm{E}+06$

$7.00 \mathrm{E}+05$

$4.87 E+05$

$3.41 E+05$

$2.40 \mathrm{E}+05$

$1.69 \mathrm{E}+05$

$1.19 \mathrm{E}+05$

8.36E+04

$5.85 \mathrm{E}+04$

$4.05 \mathrm{E}+04$

$2.82 \mathrm{E}+04$

$1.94 \mathrm{E}+04$

$1.29 E+04$

$8.58 \mathrm{E}+03$

$5.64 E+03$

$3.38 \mathrm{E}+03$

$1.56 \mathrm{E}+03$

$3.91 \mathrm{E}+01$

$6.07 \mathrm{E}+00$

$3.53 \mathrm{E}+00$

$2.07 \mathrm{E}+00$

1. $21 \mathrm{E}+00$

6.06E-01

2.64E-01

4. $19 \mathrm{E}-02$
$800 \mathrm{mil}$

Al Shield

2. $10 \mathrm{E}+08$

$1.88 \mathrm{E}+08$

$1.67 \mathrm{E}+08$

$1.39 \mathrm{E}+08$

$1.23 \mathrm{E}+08$

$1.08 \mathrm{E}+08$

$8.59 \mathrm{E}+07$

$6.74 \mathrm{E}+07$

$5.69 \mathrm{E}+07$

$4.77 \mathrm{E}+07$

$4.11 \mathrm{E}+07$

$3.50 \mathrm{E}+07$

$2.85 \mathrm{E}+07$

$2.33 E+07$

$1.84 \mathrm{E}+07$

$1.52 \mathrm{E}+07$

$1.30 \mathrm{E}+07$

$1.13 E+07$

9.79E+06

$8.54 \mathrm{E}+06$

$7.31 \mathrm{E}+06$

$5.21 \mathrm{E}+06$

$2.95 \mathrm{E}+06$

$1.89 \mathrm{E} \div 06$

$1.26 \mathrm{E}+06$

$8.61 E+05$

$5.93 E \div 05$

$4.12 \mathrm{E} \div 05$

$2.87 \mathrm{E} \div 05$

2.02E $\div 05$

$1.42 \mathrm{E}+05$

$9.97 \mathrm{E} \div 04$

$7.00 \mathrm{E}+04$

4. $8 \mathrm{SE}+04$

$3.37 \mathrm{E} \div 04$

$2.35 \mathrm{E} \div 04$

$1.61 E \div 04$

$1.07 \mathrm{E} \div 04$

$7.07 \mathrm{E} \div 03$

4.62E $\div 03$

$2.75 E \div 03$

$1.26 E \div 03$

$3.07 \mathrm{E} \div 01$

$4.42 E \div 00$

$2.54 \mathrm{E} \div 00$

$1.4 S E \div 00$

S.53E-01

4. $23 \mathrm{E}-01$

$1.83 E-01$

$2.59 \mathrm{E}-02$
$1600 \mathrm{mil}$

Al Shield

$1.73 E+08$

$1.59 \mathrm{E}+08$

$1.44 E+08$

$1.21 \mathrm{E}+08$

$1.08 \mathrm{E}+08$

$9.57 \mathrm{E}+07$

$7.60 \mathrm{E}+07$

$5.93 \mathrm{E}+07$

$5.03 \mathrm{E}+07$

$4.22 \mathrm{E}+07$

$3.64 \mathrm{E}+07$

$3.10 \mathrm{E}+07$

$2.52 \mathrm{E}+07$

$2.05 \mathrm{E}+07$

$1.60 \mathrm{E}+07$

$1.33 \mathrm{E}+07$

$1.13 \mathrm{E}+07$

$9.83 \mathrm{E}+06$

$8.49 \mathrm{E}+06$

$7.39 \mathrm{E}+06$

$6.30 \mathrm{E}+06$

$4.39 E+06$

2. $39 \mathrm{E}+06$

$1.50 \mathrm{E}+06$

$9.85 \mathrm{E}+05$

$6.65 E+05$

4.55E+05

$3.15 E+05$

2.19E+05

1.53E+05

$1.08 \mathrm{E}+05$

$7.56 \mathrm{E}+04$

$5.29 \mathrm{E}+04$

$3.68 \mathrm{E}+04$

$2.53 \mathrm{E}+04$

$1.76 \mathrm{E}+04$

$1.20 \mathrm{E}+04$

$7.94 \mathrm{E}+03$

$5.22 E+03$

$3.40 \mathrm{E}+03$

$2.00 E+03$

$9.09 \mathrm{E}+02$

$2.15 \mathrm{E}+01$

$2.85 E+00$

$1.61 E+00$

9.23E-01

$5.29 \mathrm{E}-01$

2.59E-01

$1.11 \mathrm{E}-01$

1.74E-02 
Table 7: LET Fluence Spectra for Geographos Period

MeV $/$ $\left(\mathrm{mg} / \mathrm{cm}^{2}\right)$

\begin{tabular}{ll}
0.043 & $5.67 \mathrm{E}+13$ \\
0.050 & $5.63 \mathrm{E}+13$ \\
0.059 & $5.57 \mathrm{E}+13$ \\
0.068 & $5.47 \mathrm{E}+13$ \\
0.080 & $5.32 \mathrm{E}+13$ \\
0.093 & $5.10 \mathrm{E}+13$ \\
0.109 & $4.78 \mathrm{E}+13$ \\
0.127 & $4.35 \mathrm{E}+13$ \\
0.148 & $3.84 \mathrm{E}+13$ \\
0.173 & $3.21 \mathrm{E}+13$ \\
0.202 & $2.61 \mathrm{E}+13$ \\
0.236 & $2.06 \mathrm{E}+13$ \\
0.276 & $1.60 \mathrm{E}+13$ \\
0.322 & $1.24 \mathrm{E}+13$ \\
0.377 & $9.37 \mathrm{E}+12$ \\
0.440 & $3.73 \mathrm{E}+12$ \\
0.513 & $3.01 \mathrm{E}+12$ \\
0.600 & $2.75 \mathrm{E}+12$ \\
0.700 & $2.38 \mathrm{E}+12$ \\
0.818 & $1.98 \mathrm{E}+12$ \\
0.955 & $1.56 \mathrm{E}+12$ \\
1.115 & $1.14 \mathrm{E}+12$ \\
1.302 & $6.99 \mathrm{E}+11$ \\
1.521 & $1.81 \mathrm{E}+10$ \\
1.776 & $1.78 \mathrm{E}+10$ \\
2.074 & $1.75 \mathrm{E}+10$ \\
2.422 & $1.72 \mathrm{E}+10$ \\
2.829 & $1.67 \mathrm{E}+10$ \\
3.303 & $1.62 \mathrm{E}+10$ \\
3.858 & $1.55 \mathrm{E}+10$ \\
4.505 & $1.46 \mathrm{E}+10$ \\
5.261 & $1.30 \mathrm{E}+10$ \\
6.144 & $1.13 \mathrm{E}+10$ \\
7.174 & $9.19 \mathrm{E}+09$ \\
8.378 & $7.03 \mathrm{E}+09$ \\
9.784 & $6.18 \mathrm{E}+09$ \\
11.426 & $5.46 \mathrm{E}+09$ \\
13.343 & $2.76 \mathrm{E}+09$ \\
15.582 & $2.04 \mathrm{E}+09$ \\
18.197 & $1.55 \mathrm{E}+09$ \\
21.250 & $1.09 \mathrm{E}+09$ \\
24.816 & $6.36 \mathrm{E}+08$ \\
28.980 & $1.52 \mathrm{E}+07$ \\
33.843 & $5.97 \mathrm{E}+05$ \\
39.522 & $2.31 \mathrm{E}+05$ \\
46.153 & $8.93 \mathrm{E}+04$ \\
53.898 & $3.31 \mathrm{E}+04$ \\
62.942 & $9.57 \mathrm{E}+03$ \\
73.503 & $2.26 \mathrm{E}+03$ \\
85.837 & $1.73 \mathrm{E}+02$ \\
& \\
\hline
\end{tabular}

$100 \mathrm{mil} \quad 200 \mathrm{mil}$

Al Shield Al Shield

$5.28 \mathrm{E}+09$

$3.97 \mathrm{E}+09$

$2.95 \mathrm{E}+09$

2.17E+09

$1.59 \mathrm{E}+09$

$1.17 \mathrm{E}+09$

$8.43 E+08$

$6.02 \mathrm{E}+0.8$

$4.37 \mathrm{E}+08$

$3.09 \mathrm{E}+08$

$2.25 \mathrm{E}+08$

$1.64 \mathrm{E}+08$

$1.19 \mathrm{E}+08$

$8.79 \mathrm{E}+07$

$6.28 \mathrm{E}+07$

$3.11 \mathrm{E}+07$

$2.41 \mathrm{E}+07$

$2.06 \mathrm{E}+07$

$1.75 E+07$

$1.51 \mathrm{E}+07$

$1.28 \mathrm{E}+07$

$9.35 E+06$

$5.59 \mathrm{E}+06$

$3.58 \mathrm{E}+06$

$2.46 \mathrm{E}+06$

$1.72 \mathrm{E}+06$

$1.21 \mathrm{E}+06$

$8.51 \mathrm{E}+05$

$6.03 \mathrm{E}+05$

$4.29 \mathrm{E}+05$

$3.06 \mathrm{E}+05$

$2.18 E+05$

$1.55 \mathrm{E}+05$

$1.09 \mathrm{E}+05$

$7.62 E+04$

$5.36 \mathrm{E}+04$

$3.73 \mathrm{E}+04$

$2.51 E+04$

1. $68 \mathrm{E}+04$

1. $12 E+04$

$6.83 \mathrm{E}+03$

3. $26 \mathrm{E}+03$

$8.60 E+01$

$1.24 \mathrm{E}+01$

$7.24 \mathrm{E}+00$

$4.29 \mathrm{E}+00$

$2.51 \mathrm{E}+00$

$1.27 \mathrm{E}+00$

$5.59 \mathrm{E}-01$

8.97E-02
$1.29 \mathrm{E}+09$

$9.88 \mathrm{E}+08$

$7.57 \mathrm{E}+08$

$5.73 \mathrm{E}+08$

$4.43 \mathrm{E}+08$

$3.45 \mathrm{E}+08$

$2.58 \mathrm{E}+08$

$1.92 \mathrm{E}+08$

$1.49 \mathrm{E}+08$

$1.15 \mathrm{E}+08$

$9.14 \mathrm{E}+07$

$7.30 \mathrm{E}+07$

$5.72 \mathrm{E}+07$

$4.52 \mathrm{E}+07$

$3.47 \mathrm{E}+07$

$2.51 \mathrm{E}+07$

$2.10 \mathrm{E}+07$

$1.82 \mathrm{E}+07$

$1.58 \mathrm{E}+07$

$1.37 \mathrm{E}+07$

1.18E+07

$8.63 E+06$

$5.11 \mathrm{E}+06$

$3.36 \mathrm{E}+06$

2.29E+06

$1.59 \mathrm{E}+06$

$1.11 \mathrm{E}+06$

$7.74 \mathrm{E}+05$

$5.43 E+05$

$3.83 \mathrm{E}+05$

$2.71 \mathrm{E}+05$

$1.91 \mathrm{E}+05$

$1.34 \mathrm{E}+05$

$9.42 \mathrm{E}+04$

$6.53 \mathrm{E}+04$

$4.56 \mathrm{E}+04$

$3.15 \mathrm{E}+04$

2.10E+04

$1.40 \mathrm{E}+04$

$9.23 E+03$

$5.57 \mathrm{E}+03$

$2.60 \mathrm{E}+03$

$6.67 \mathrm{E}+01$

$1.07 \mathrm{E}+01$

$6.25 \mathrm{E}+00$

$3.70 \mathrm{E}+00$

$2.16 \mathrm{E}+00$

$1.09 \mathrm{E}+00$

4.79E-01

7.66E-02
$400 \mathrm{mil}$

Al Shield

$4.70 \mathrm{E}+08$

$3.92 \mathrm{E}+08$

$3.27 \mathrm{E}+08$

$2.63 \mathrm{E}+08$

$2.22 \mathrm{E}+08$

$1.87 \mathrm{E}+08$

$1.47 \mathrm{E}+08$

$1.14 \mathrm{E}+08$

9.44E+07

$7.76 \mathrm{E}+07$

$6.57 \mathrm{E}+07$

$5.52 \mathrm{E}+07$

$4.48 \mathrm{E}+07$

$3.65 \mathrm{E}+07$

$2.88 \mathrm{E}+07$

$2.32 E+07$

$1.98 \mathrm{E}+07$

$1.72 \mathrm{E}+07$

$1.49 \mathrm{E}+07$

$1.30 \mathrm{E}+07$

$1.11 E+07$

$8.10 \mathrm{E}+06$

$4.73 E+06$

$3.09 \mathrm{E}+06$

$2.09 \mathrm{E}+06$

$1.44 \mathrm{E}+06$

$9.99 \mathrm{E}+05$

$6.96 \mathrm{E}+05$

$4.88 \mathrm{E}+05$

$3.43 E+05$

$2.42 E+05$

$1.70 \mathrm{E}+05$

$1.20 \mathrm{E}+05$

$8.36 \mathrm{E}+04$

$5.79 E+04$

$4.03 \mathrm{E}+04$

$2.78 E+04$

$1.85 \mathrm{E}+04$

$1.23 \mathrm{E}+04$

$8.06 \mathrm{E}+03$

$4.83 \mathrm{E}+03$

$2.23 \mathrm{E}+03$

$5.59 E+01$

8.61E+00

$5.00 \mathrm{E}+00$

$2.94 \mathrm{E}+00$

$1.71 \mathrm{E}+00$

8.57E-01

3.73E-01

5.93E-02
$800 \mathrm{mil}$ Al Shield

$2.93 E+08$

$2.63 \mathrm{E}+08$

$2.33 \mathrm{E}+08$

$1.95 \mathrm{E}+08$

$1.72 \mathrm{E}+08$

$1.51 \mathrm{E}+08$

$1.20 \mathrm{E}+08$

9.45E+07

$7.98 \mathrm{E}+07$

$6.69 \mathrm{E}+07$

$5.76 \mathrm{E}+07$

$4.90 \mathrm{E}+07$

$3.99 \mathrm{E}+07$

$3.27 \mathrm{E}+07$

$2.58 \mathrm{E}+07$

2.13E+07

$1.82 \mathrm{E}+07$

$1.58 \mathrm{E}+07$

$1.37 \mathrm{E}+07$

$1.19 \mathrm{E}+07$

$1.02 E+07$

$7.30 \mathrm{E}+06$

4. $15 E+06$

2. $67 \mathrm{E}+06$

$1.79 \mathrm{E}+06$

$1.22 \mathrm{E}+06$

8. $41 E+05$

$5.84 \mathrm{E}+05$

$4.08 E+05$

$2.86 \mathrm{E}+05$

$2.01 \mathrm{E}+05$

$1.42 \mathrm{E}+05$

$9.93 \mathrm{E} \div 04$

$6.93 \mathrm{E} \div 04$

$4.78 \mathrm{E} \div 04$

$3.33 \mathrm{E} \div 04$

2. $29 \mathrm{E} \div 04$

$1.51 E \div 04$

$1.00 E+04$

$6.55 \mathrm{E} \div 03$

$3.89 \mathrm{E} \div 03$

1.7SE $\div 03$

4. $35 \mathrm{E} \div 01$

$6.23 E+00$

$3.56 \mathrm{E} \div 00$

$2.07 \mathrm{E} \div 00$

$1.20 \mathrm{E}+00$

$5.94 \mathrm{E}-01$

$2.57 \mathrm{E}-01$

$4.05 \mathrm{E}-02$
$1600 \mathrm{mil}$

Al Shield

$2.41 E+08$

$2.22 \mathrm{E}+08$

$2.01 \mathrm{E}+08$

$1.69 \mathrm{E}+08$

$1.51 \mathrm{E}+08$

$1.34 \mathrm{E}+08$

$1.06 \mathrm{E}+08$

8.31E $\div 07$

$7.03 \mathrm{E}+07$

$5.90 \mathrm{E}+07$

$5.09 \mathrm{E}+07$

4.32E +07

$3.51 \mathrm{E}+07$

$2.85 \mathrm{E}+07$

$2.24 \mathrm{E}+07$

$1.85 \mathrm{E}+07$

$1.58 \mathrm{E}+07$

$1.37 \mathrm{E}+07$

$1.18 E+07$

$1.03 \mathrm{E}+07$

$8.75 E+06$

$6.11 E+06$

$3.34 \mathrm{E}+06$

2.10E+06

$1.39 E+06$

$9.37 \mathrm{E}+05$

$6.41 E+05$

4. $44 \mathrm{E}+05$

$3.09 \mathrm{E}+05$

$2.16 \mathrm{E}+05$

$1.52 \mathrm{E}+05$

$1.07 \mathrm{E}+05$

$7.45 \mathrm{E}+04$

$5.19 \mathrm{E}+04$

$3.57 \mathrm{E}+04$

$2.47 \mathrm{E}+04$

$1.69 \mathrm{E}+04$

1.12E+04

$7.34 \mathrm{E}+03$

$4.78 \mathrm{E}+03$

$2.81 E+03$

$1.28 \mathrm{E}+03$

$3.02 \mathrm{E}+01$

$3.97 \mathrm{E}+00$

$2.24 \mathrm{E}+00$

$1.29 \mathrm{E}+00$

7.36E-01

3.61E-01

$1.55 \mathrm{E}-01$

2.42E-02 
For the ISA, the effects of the Earth's magnetic field must be taken into account. We have applied the geomagnetic transmission function discussed earlier (Section IV.D) to the SEP, GCR and AC components and used the result to determine LET spectra appropriate for the ISA orbit. An example of these results is given in Figure 38, which shows the LET fluence spectra for the "Typical" SEP sample (top) and the LET flux spectra for the GCR and AC combination. The curves in these figures can be directly compared to the results shown in Figures 34 and 35 which do include geomagnetic transmission effects. No substantial difference can be found. This is not a surprising result as we have previously seen that, due to the highly eccentric ISA orbit, the average radiation environment experienced by the ISA differs from the interplanetary space environment only by a small amount at low energy.

The LET fluence spectra calculated for the Interstage Adapter for the time period from 2/1/94 to 10/1/94 is shown in Figure 39 for a 1 micron Si sensitive layer and Al shields of thickness 50, 100, 200, 400, 800 and 1600 mils. The particle flux used in this calculation includes galactic cosmic rays modulated to $\phi=570$ $M V$, Anomalous Components and the "Typical" SEP sample appropriately scaled for this time period. The spectra of these energetic particles were corrected for the effects of geomagnetic transmission prior to calculating the LET spectra. A listing of the LET spectra is provided in Table 8.

\section{CONCLUSIONS}

We have used a new model of the space radiation environment, CHIME, recently developed for the CRRES/SPACERAD mission to predict the environment and derive LET spectra for the CLEMENTINE I mission. The CHIME model represents an advance in the accuracy of estimating radiation environments as it is based upon the physical theory of cosmic ray interstellar and heliospheric propagation and is "calibrated" with energy spectra and ratio measurements accumulated over the last two decades. This model provided the predictions for the galactic cosmic ray (GCR) and anomalous component (AC) fluxes during 1994. The remaining major component of the interplanetary radiation environment, the solar energetic particles, were estimated by combining SEP events observed during the CRRES mission into sample populations which are consistent with historical variations.

The CHIME model predicts that during 1994 solar aci:-ill be decreasing toward solar minimum levels, and the galactic cosmic ray and anomalous component fluxes will be increasing. During the early Lunar Survey phase of the mission, the solar modulation level is expected to be about $58 S M V$, while later in the mission during the asteroid flyby, the modulation level will decrease to $558 \mathrm{MV}$. The average modulation level over the entire mission is predicted to be $570 \mathrm{MV}$. These levels have an uncertainty of about 40 to $50 \mathrm{MV}$ and can be 

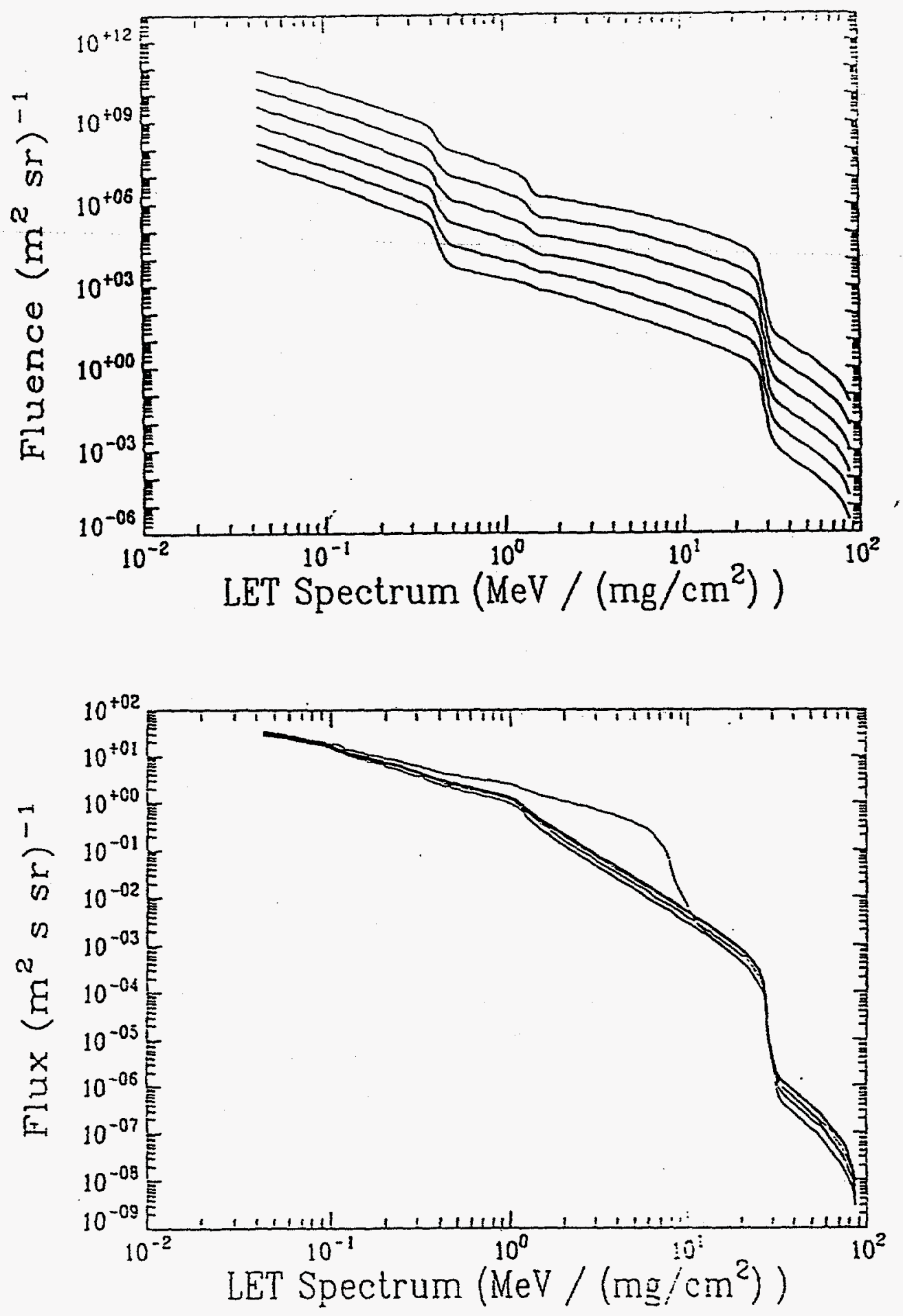

Figure 38. The LET for the "Typical" SEP sample (top) ard Galactic Cosmic Rays with Anomalous species (bottom) calculated for the Interstage Adapter and including the effects of geomagnetic transmission. There is no substantial difference between these plots and those calculated using interplanetary spectra. 


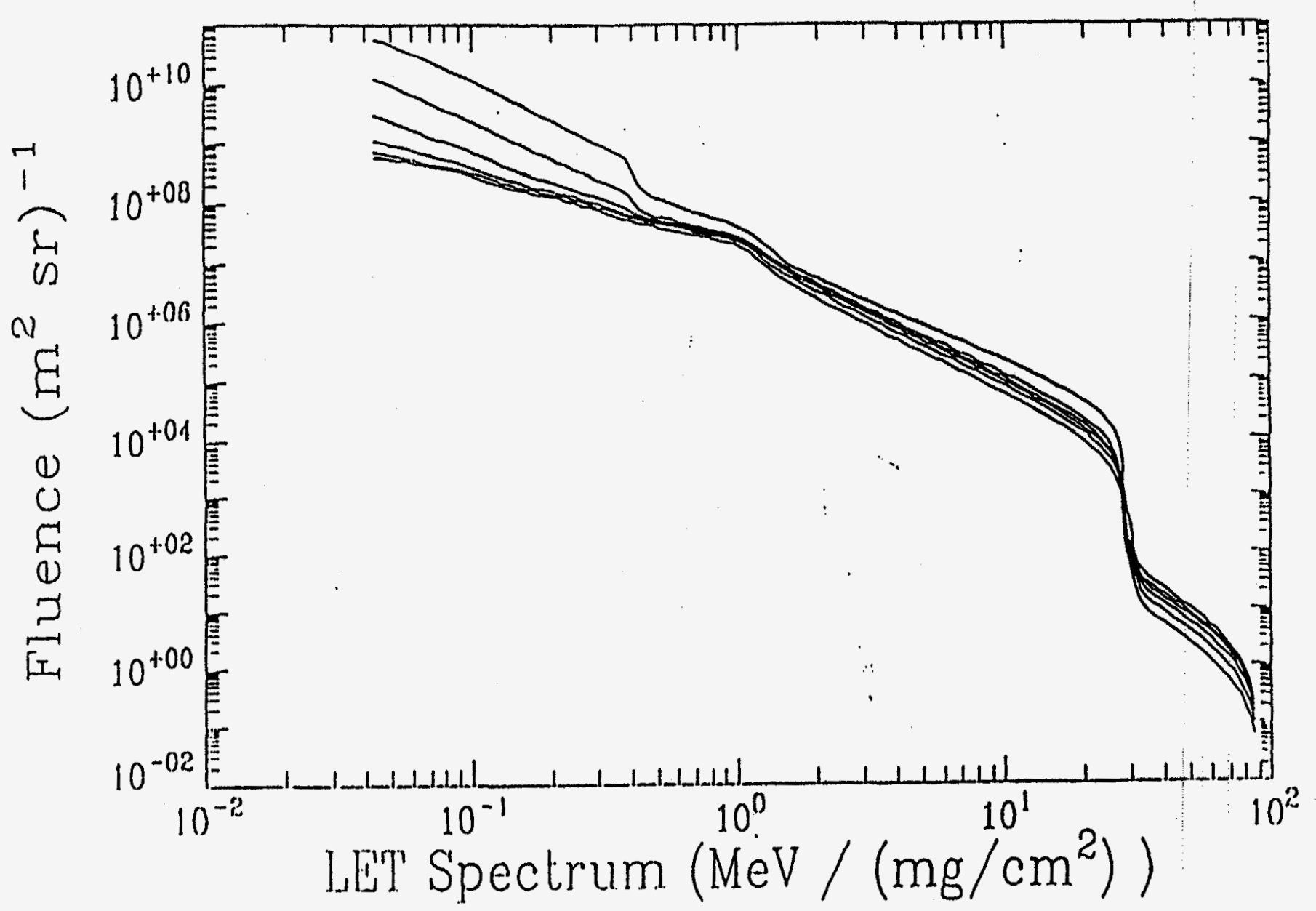

Figure 39: The LE'r fluence spectrum calculated for the Interstage Adapter for the time period from 2/1/94 10 10/1/94 is shown for a 1 micron Silicon sensitive layer and Aluminum shields of thickness 50, 100, 200, 400,800 , and 1600 mils. The particle flux used in this calculation includes Galactic Cosmic Rays, Anomalous species and the "Typical" SEP sample fluence scaled for the time period. The level of solar modulation used was $\$-570 \mathrm{MV}$. 
Table 8: LET Fluence Spectra for Interstage Adapter

$\mathrm{MeV} /$ $\left(\mathrm{mg} / \mathrm{cm}^{2}\right.$ )

$\begin{array}{lll}0.043 & 5.94 E+10 & 1.28 E+10 \\ 0.050 & 4.60 E+10 & 9.64 E+09 \\ 0.059 & 3.48 E+10 & 7.16 E+09 \\ 0.068 & 2.59 E+10 & 5.27 E+09 \\ 0.080 & 1.90 E+10 & 3.87 E+09 \\ 0.093 & 1.39 E+10 & 2.84 E+09 \\ 0.109 & 9.99 E+09 & 2.05 E+09 \\ 0.127 & 7.11 E+09 & 1.46 E+09 \\ 0.148 & 5.09 E+09 & 1.06 E+09 \\ 0.173 & 3.51 E+09 & 7.52 E+08 \\ 0.202 & 2.46 E+09 & 5.48 E+08 \\ 0.236 & 1.72 E+09 & 4.00 E+08 \\ 0.276 & 1.20 E+09 & 2.91 E+08 \\ 0.322 & 8.37 E+08 & 2.14 E+08 \\ 0.377 & 5.65 E+08 & 1.53 E+08 \\ 0.440 & 1.74 E+08 & 7.61 E+07 \\ 0.513 & 1.11 E+08 & 5.91 E+07 \\ 0.600 & 8.86 E+07 & 5.04 E+07 \\ 0.700 & 7.00 E+07 & 4.29 E+07 \\ 0.818 & 5.64 E+07 & 3.69 E+07 \\ 0.955 & 4.50 E+07 & 3.1 .3 E+07 \\ 1.115 & 3.23 E+07 & 2.29 E+07 \\ 1.302 & 1.94 E+07 & 1.36 E+07 \\ 1.521 & 1.01 E+07 & 8.71 E+06 \\ 1.776 & 7.18 E+06 & 5.99 E+06 \\ 2.074 & 5.15 E+06 & 4.18 E+06 \\ 2.422 & 3.72 E+06 & 2.93 E+06 \\ 2.829 & 2.71 E+06 & 2.07 E+06 \\ 3.303 & 1.99 E+06 & 1.46 E+06 \\ 3.858 & 1.47 E+06 & 1.04 E+06 \\ 4.505 & 1.09 E+06 & 7.43 E+05 \\ 5.261 & 8.07 E+05 & 5.30 E+05 \\ 6.144 & 5.94 E+05 & 3.77 E+05 \\ 7.174 & 4.31 E+05 & 2.66 E+05 \\ 8.378 & 3.07 E+05 & 1.85 E+05 \\ 9.784 & 2.25 E+05 & 1.30 E+05 \\ 11.426 & 1.63 E+05 & 9.08 E+04 \\ 13.343 & 1.13 E+05 & 6.10 E+04 \\ 15.582 & 7.83 E+04 & 4.10 E+04 \\ 18.197 & 5.36 E+04 & 2.72 E+04 \\ 21.250 & 3.40 E+04 & 1.67 E+04 \\ 24.816 & 1.73 E+04 & 7.95 E+03 \\ 28.980 & 4.69 E+02 & 2.09 E+02 \\ 33.843 & 3.96 E+01 & 3.01 E+01 \\ 39.522 & 2.20 E+01 & 1.76 E+01 \\ 46.153 & 1.27 E+01 & 1.04 E+01 \\ 53.898 & 7.41 E+00 & 6.09 E+00 \\ 62.942 & 3.69 E+00 & 3.09 E+00 \\ 73.503 & 1.61 E+00 & 1.35 E+00 \\ 85.837 & 2.58 E-01 & 2.17 E-01\end{array}$

$200 \mathrm{mil}$ Al Shield

$3.15 \mathrm{E}+09$

$2.42 \mathrm{E}+09$

$1.85 \mathrm{E}+09$

$1.40 \mathrm{E}+09$

$1.08 \mathrm{E}+09$

$8.44 \mathrm{E}+08$

$6.32 \mathrm{E}+08$

$4.69 \mathrm{E}+08$

$3.65 \mathrm{E}+08$

$2.81 \mathrm{E}+08$

$2.24 \mathrm{E}+08$

$1.79 \mathrm{E}+08$

$1.40 \mathrm{E}+08$

$1.11 \mathrm{E}+08$

$8.51 \mathrm{E}+07$

$6.14 \mathrm{E}+07$

$5.15 \mathrm{E}+07$

4.47E+07

$3.87 \mathrm{E}+07$

$3.37 \mathrm{E}+07$

$2.89 \mathrm{E}+07$

$2.11 \mathrm{E}+07$

$1.25 \mathrm{E}+07$

$8.18 \mathrm{E}+06$

$5.58 \mathrm{E}+06$

$3.86 \mathrm{E}+06$

$2.69 \mathrm{E}+06$

$1.88 \mathrm{E}+06$

$1.32 \mathrm{E}+06$

$9.29 \mathrm{E}+05$

$6.56 \mathrm{E}+05$

$4.63 E+05$

$3.26 \mathrm{E}+05$

$2.29 \mathrm{E}+05$

$1.59 \mathrm{E}+05$

$1.11 E+05$

$7.66 \mathrm{E}+04$

$5.11 \mathrm{E}+04$

$3.41 \mathrm{E}+04$

$2.25 \mathrm{E}+04$

1.36E+04

$6.32 \mathrm{E}+03$

$1.62 \mathrm{E}+02$

$2.60 \mathrm{E}+01$

$1.52 \mathrm{E}+01$

$9.02 \mathrm{E}+00$

$5.28 \mathrm{E}+00$

$2.67 \mathrm{E}+00$

$1.17 \mathrm{E}+00$

1.87E-01
$400 \mathrm{mil}$

Al Shield

$1.16 \mathrm{E}+09$

9.64E+08

$8.03 E+08$

$6.46 \mathrm{E}+08$

$5.45 \mathrm{E}+08$

$4.60 \mathrm{E}+08$

$3.61 \mathrm{E}+08$

$2.80 \mathrm{E}+08$

$2.31 E+08$

$1.90 \mathrm{E}+08$

$1.61 \mathrm{E}+08$

$1.35 \mathrm{E}+08$

$1.10 \mathrm{E}+08$

8.94E+07

$7.05 \mathrm{E}+07$

$5.68 \mathrm{E}+07$

$4.85 \mathrm{E}+07$

4.22E +07

$3.65 \mathrm{E}+07$

$3.19 E+07$

$2.74 \mathrm{E}+07$

$1.98 \mathrm{E}+07$

$1.16 \mathrm{E}+07$

$7.53 E+06$

$5.10 \mathrm{E}+06$

$3.51 \mathrm{E}+06$

$2.43 E+06$

$1.70 E+06$

$1.19 \mathrm{E}+06$

$8.34 E+05$

$5.88 E+05$

$4.14 \mathrm{E}+05$

$2.91 E+05$

$2.04 \mathrm{E}+05$

$1.41 \mathrm{E}+05$

$9.82 E+04$

$6.76 \mathrm{E}+04$

$4.50 E+04$

$2.99 E+04$

$1.96 \mathrm{E}+04$

$1.18 \mathrm{E}+04$

$5.43 E+03$

$1.36 \mathrm{E}+02$

$2.11 E+01$

$1.22 \mathrm{E}+0.1$

7. $19 \mathrm{E}+00$

$4.19 \mathrm{E}+00$

$2.10 \mathrm{E}+00$

9.15E-01

$1.46 \mathrm{E}-01$
$800 \mathrm{mil}$

Al Shield

$7.18 \mathrm{E}+08$

$6.44 \mathrm{E}+08$

$5.71 E+08$

4.77E+08

4.22E+08

$3.70 \mathrm{E}+08$

$2.95 \mathrm{E}+08$

$2.32 \mathrm{E}+08$

$1.96 \mathrm{E}+08$

$1.64 \mathrm{E}+08$

$1.41 \mathrm{E}+08$

$1.20 \mathrm{E}+08$

$9.80 \mathrm{E}+07$

$8.01 E+07$

$6.33 E+07$

$5.22 \mathrm{E}+07$

$4.47 \mathrm{E}+07$

$3.88 \mathrm{E}+07$

$3.35 \mathrm{E}+07$

2. $93 \mathrm{E}+07$

$2.50 \mathrm{E}+07$

$1.79 E+07$

$1.02 E \div 07$

$6.52 E+06$

4. $36 E+06$

$2.98 E+06$

$2.05 \mathrm{E}+06$

$1.43 E+06$

$9.96 \mathrm{E}+05$

$6.99 E \div 05$

$4.91 E+05$

$3.45 E \div 05$

$2.42 \mathrm{E} \div 05$

$1.69 E \div 05$

1.17E $\div 05$

$8.12 E \div 04$

$5.58 \mathrm{E} \div 04$

3. $70 \mathrm{E} \div 04$

$2.45 \mathrm{E} \div 04$

1. $60 \mathrm{E} \div 04$

$9.51 \mathrm{E} \div 03$

$4.35 \mathrm{E} \div 03$

$1.06 E \div 02$

$1.5 \geq E \div 01$

$8.72 E+00$

$5.08 E \div 00$

$2.93 E \div 00$

$1.46 \mathrm{E}+00$

6.30E-01

9.95E-02
$1600 \mathrm{mil}$

Al Shield

$5.92 \mathrm{E}+08$

$5.46 \mathrm{E}+08$

$4.93 \mathrm{E}+08$

4.15E+08

$3.71 \mathrm{E}+08$

$3.28 \mathrm{E}+08$

$2.61 \mathrm{E}+08$

$2.04 E+08$

$1.73 E+08$

$1.45 \mathrm{E}+08$

$1.25 \mathrm{E}+08$

$1.06 \mathrm{E}+08$

$8.62 \mathrm{E}+07$

$7.01 \mathrm{E}+07$

$5.50 \mathrm{E}+07$

$4.54 E+07$

$3.88 \mathrm{E}+07$

$3.36 \mathrm{E}+07$

$2.90 \mathrm{E}+07$

$2.53 \mathrm{E}+07$

$2.15 \mathrm{E}+07$

$1.50 \mathrm{E}+07$

$8.20 \mathrm{E}+06$

$5.15 E+06$

$3.39 E+06$

$2.29 E+06$

$1.57 \mathrm{E}+06$

$1.08 \mathrm{E}+06$

$7.54 \mathrm{E}+05$

$5.28 E+05$

$3.71 E+05$

$2.60 E+05$

$1.82 \mathrm{E}+05$

1.27E+05

$8.72 E+04$

$6.05 E+04$

4.14E+04

$2.73 E+04$

$1.80 \mathrm{E}+04$

1.17E+04

$6.88 E+03$

$3.13 E+03$

$7.42 E+0.1$

$9.76 \mathrm{E}+00$

$5.50 E+00$

3. $16 \mathrm{E}+00$

$1.81 \mathrm{E}+00$

$8.86 \mathrm{E}-01$

3.80E-01

5.94E-02 
compared to a typical solar minimum level of $475 \mathrm{MV}$ and solar maximum level of $1500 \mathrm{MV}$.

Modulated GCR and AC spectra were combined and used to derive a linear energy transfer (LET) spectra. It was found that the AC dominates the particle spectra below about $30-50 \mathrm{MeV} /$ nucleon and due to their steep energy spectra can be effectively shielded by relatively small $(\sim 50$ mils of $\mathrm{Al})$ amounts of material. The effects of the AC may be observed in unshielded detectors/ components. The GCR, however, are highly penetrating and require massive amounts of shielding ( $>50 \mathrm{~g} / \mathrm{cm}^{2}$ ) before a significant reduction in the LET spectra flux is achieved.

By examining the historical number and intensity variations, it is estimated that about 10 \pm 5 SEP events might be observed by the CLEMENTINE I spacecraft during 1994. The total fluence of $>10 \mathrm{MeV}$ protons for these events should be less than $10^{8}$ particles $/\left(\mathrm{cm}^{2} \mathrm{sr}\right)$, though there is a chance for a large event with a fluence exceeding $10^{9}$ particles $/\left(\mathrm{cm}^{2} \mathrm{sr}\right)$. Four sample SEP populations, consistent with number and proton fluence estimates, were constructed from the 26 events characterized during the CRRES/SPACERAD mission. These sample populations differed slightly in their composition and included: 1) Typical composition, 2) Heavy Ion Rich composition, 3) Heavy Ion Poor composition and 4) Typical composition with a single large event. LET spectra were calculated for each of these samples. Slight differences were found for samples $1-3$ for LET $>3-4 \mathrm{MeV} /\left(\mathrm{mg} / \mathrm{cm}^{2}\right)$ due to differences in the heavy ion content, while the single large event in sample 4 resulted in significant fluence enhancements for all LET values. However, due to the relatively steep energy spectra of most SEP events, moderate amounts of material (100-300 mils of Al) can effectively shield against these particles.

LET spectra for the two CLEMENTINE I spacecraft phases (lunar orbit and asteroid flyby) and the interstage adapter (ISA) were calculated by combining the GCR, AC and "typical" composition SEP fluxes and, for the ISA, taking into account the effect of geomagnetic transmission. For each of these cases it was found that the GCR dominate the LET spectra for LET greater than about 1 $\mathrm{MeV} /\left(\mathrm{mg} / \mathrm{cm}^{2}\right)$. Below this level the SEP effects can be seen for $\mathrm{Al}$ shields less than 300 mils thick. The geomagnetic transmission effects averaged over the highly eccentric ISA orbit are negligible, resulting in, at most a $7 \%$ decrease in the proton flux at $10 \mathrm{MeV}$. Thus, even taking these geomagnetic effects into account results in LET spectra for the ISA which are essentially identical to those for interplanetary space. 
This independent estimate of the CLEMENTINE-I radiation environment can be compared to previous estimates and to the results derived from the sensors, electronic components and radiation measuring devices. The authors would be interested in receiving copies of any such comparisons that are performed.

The CHIME model used in this study was developed with the support of the U.S. Air Force and the Office of Naval Research. In addition, one of us, E. Clayton, participated in this study with the additional support of the Louisiana Board of Regents, LEQSF, under agreement NASA/LSU-(91-96)-01, and NASA/LaSPACE under grant NGT-40039. 
VI. Bibliography

Binder, D., Smith, E. C., and Holman, A. B. (1975), "Satellite Anomalies from Galactic Cosmic Rays", IEEE Trans. Nucl. Sci., NS-22, 2675.

Biswas, S., Durgaprasad, N., Mitra, B., and Dutta, A. (1993), Space Science Reviews, 62, 3 .

Chen, J., Chenette, D., Clark, R., Garcia-Munoz, M., Guzik, T. G., Pyle, K., Sang, Y. and Wefel, J. (1994a), "A model of galactic cosmic rays for use in calculating linear energy transfer spectra", Adv. Sp. Res., 14, No. 10, 765.

Chen, J., Chenette, D., Garcia-Munoz, M., Guzik, T. G., Pyle, K., Sang, Y. and Wefel, J. (1994b), "A model of solar energetic particles for use in calculating LET spectra developed from ONR-604 data", Adv. Sp. Res., 14, No. 10, 675.

Chen, J., Chenette, D., Clayton, E., Garcia-Munoz, M., Guzik, T. G., Hardy, D. A., Lopate, C., Mullen, E. G., Pyle, K. R., Ray, K. P., and Wefel, J. (1994c), "The CRRES/SPACERAD Heavy Ion Model of the Environment (CHIME) for Cosmic Ray and Solar Particle Effects on Electronic and Biological Systems", IEEE Trans. Nucl. Sci., in press.

Dietrich, W. F. and Simpson, J. A. (1978), "Preferential Enhancements of the Solar Flare-Accelerated Nuclei Carbon to Zinc from -20-300 $\mathrm{MeV} /$ nucleon", Ap. J. Letters, 225, L41.

Engelmann, J. J., Ferrando, P., Soutoul, A., Goret, P., Juliusson, E., KochMiramond, L., Lund, N., Masse, P., Peters, B., Petrou, N. and Rasmusen, I. L. (1990), "Charge Composition and Energy Spectra of Cosmic-Ray Nuclei for Elements from Be to Ni. Results from HEAO-3-C2", Aston. and Astrophys., 233, 96.

Evenson, P., Garcia-Munoz, M., Meyer, P., Pyle, K. R., and Simpson, J. A. (1983), "A Quantitative Test of Solar Modulation Theory: The Proton, Helium, and Electron Spectra from 1965 through 1979", Ap. J. Letters, 275, L15.

Feynman, J., Spitale, G., Wang, J. and Gabriel, S. (1993), "Interplanetary Proton Fluence Model: JPL 1991, J. Geophys. Res., 98, 13281.

Fichtel, C. E. and Reames, D. V. (1968), "Cosmic-ray Propagation", Phys. Rev., 175,1564 .

Fisk, L. A. (1979), "The Interactions of Energetic Particles with the Solar Wind", in Solar System Plasma Physics, Vol. 1, ed. E: N. Parker, C. F. Kennel and L. J. Lanzerotti (Amsterdam: North Holland), p. 179. 
Garcia-Munoz, M., Meyer, P., Pyle, K. R., Simpson, J. A. and Evenson, P. A. (1986), "The Dependence of Solar Modulation on the Sign of the Cosmic Ray Particle Charge", J. Geophys. Res., 912858.

Garcia-Munoz, M., Simpson, J. A., Guzik, T. G., Wefel, J. P. and Margolis, S. H. (1987), "Cosmic-ray Propagation in the Galaxy and in the Heliosphere: The Pathlength Distribution at Low Energy", Ap. J. Suppl., 64, 269.

Gleeson, L. J. and Alford, W. I. (1968), "Solar Modulation of Galactic Cosmic Rays", Ap. J., $154,1011$.

Gussenhaven, M. S., Mullen, E. G., and Sagalyn, R. C. eds. (1985), CRRES/SPACERAD Experiment Descriptions, Air Force Geophysics Laboratory Report AFGL-TR-85-0017, Hanscom AFB, MA.

Guzik, T. G. (1988), "The SEP Matter Sample and its Correlation with Gammaray Observations", Solar Physics, $\underline{118}, 185$.

Heckman, H. H., Perkins, B. L., Simon, W. G., Smith, F. M. and Barkas, W. H. (1960), Phys. Rev., 117 544.

Janni, J. H. (1982), "Proton Range Energy Tables, $1 \mathrm{keV}$ - $10 \mathrm{GeV}$ ", Atomic Data and Nuclear Data Tables, 27, No. 415.

Jokipii, J. R. (1971), "Propagation of Cosmic Rays in the Solar Wind", Rev. Geophys. Space Phys., 927.

Kocharov, I. G. and Kocharov, G. E. (1984), Space Sci. Rev., 38, 89.

Kolasinski, W. A., Blake, J. B., Anthony, J. K., Price, W. E. and Smith, E. C. (1979), "Simulation of Cosmic-Ray Induced Soft Errors and Latchup in Integrated Circuit Computer Memories", IEEE Trans. Nucl. Sci., NS-26, 5087.

Kóta, J. and Jokippi, J. R. (1983), "Effects of drift on the transport of cosmic rays. VI. A three-dimensional model including diffusion", Ap. J., 265, 573.

McKibben, R. B., (1986), "Modulation of Galactic Cosmic Rays in the Heliosphere", in The Sun and the Heliosphere in Three Dimensions, ed. R. G. Marsden, (Dordrecht, The Netherlands; D. Reidel Publ. Co.), p. 361.

Melrose, D. B. (1990), Aust. J. Phys., $4 \underline{3} 703$.

Parker, E. N. (1965), "The Passage of Energetic Charged Particles Through Interplanetary Space", Plant. Space Sci., 13, 9. 
Ramaty, R., et al. (1980), Solar Flares, A Monograph from the Skvlab Solar Workshop II ed. P.A. Sturrock (Boulder: Colorado Assoc. Uinir. Press), p. 117.

Reames, D. V., Meyer, J. P., and Von Rosenvinge, T. T. (1994), Ap. J. Suppl., to be published.

Urch, 1. H. and Gleeson, L. J. (1972), "Galactic Cosmic Ray Modulation from 19651970", Astrophys. Space Sci., 17, 426.

Wilson, J. W., Townsend, L. W., Schimmerling, W., Khandelwal, G. S., Khan, F., Nealy, J. B., Cucinotta, F. A., Simonsen, L. C., Shium, J. L. and Norbury, J. W. (1991), "Transport Methods and Interactions for Space Radiations", NASA Ref. Pub. 1257, p. 475. 